
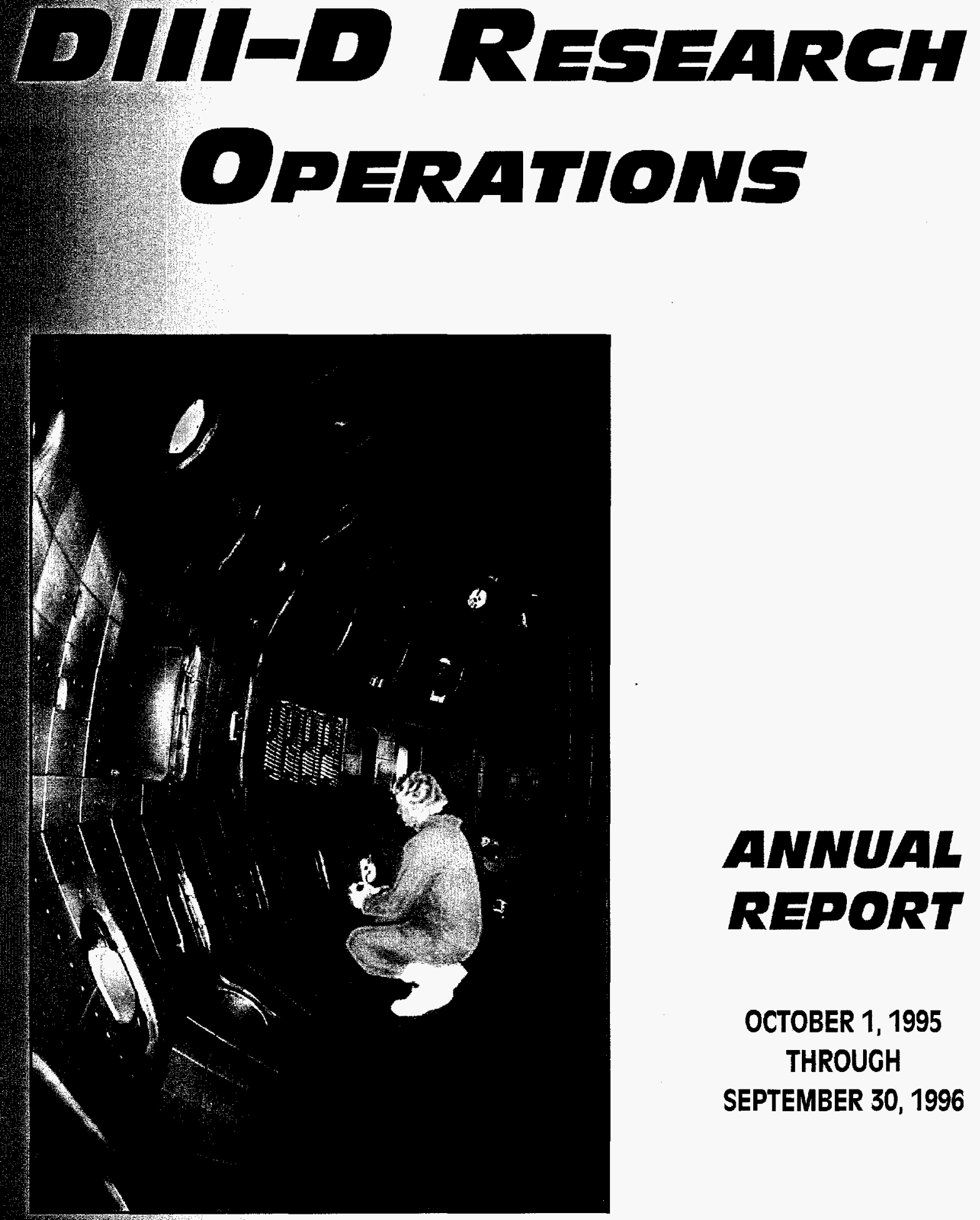

ANNUAL
REPORT

OCTOBER 1, 1995 THROUCH

SEPTEMBER 30, 1996 


\section{DISCLAIMIER}

Portions of this document may be illegible in electronic image products. Images are produced from the best available original document. 


\title{
DIII-D RESEARCH OPERATIONS ANNUAL REPORT TO THE U.S. DEPARTMENT OF ENERGY
}

OCTOBER 1, 1995 THROUGH SEPTEMBER 30, 1996

\author{
by \\ PROJECT STAFF
}

Work prepared under

Department of Energy

Contract No. DE-AC03-89ER51114

W-7405-ENG-48 and DE-AC05-960R22464

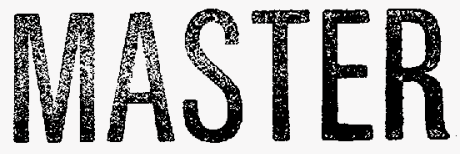

GENERAL ATOMICS PROJECTS 3466, 3467, 3470, 3473

DATE PUBLISHED: JULY 1997 


\section{DISCLAIMER}

This report was prepared as an account of work sponsored by an agency of the United States Government. Neither the United States Government nor any agency thereof, nor any of their employees, makes any warranty, express or implied, or assumes any legal liability or responsibility for the accuracy, completeness, or usefulness of any information, apparatus, produce, or process disclosed, or represents that its use would not infringe privately owned rights. Reference herein to any specific commercial product, process, or service by trade name, trademark, manufacturer, or otherwise, does not necessarily constitute or imply its endorsement, recommendation, or favoring by the United States Government or any agency thereof. The views and opinions of authors expressed herein do not necessarily state or reflect those of the United States Government or any agency thereof.

This report has been reproduced directly from the best available copy.

Available to DOE and DOE contractors from the

Office of Scientific and Technical Information

P.O. Box 62

Oak Ridge, TN 37831

Prices available from (615) 576-8401,

FTS 626-8401.

Available to the public from the

National Technical Information Service

U.S. Department of Commerce

5285 Port Royal Rd.

Springfield, VA 22161 


\section{CONTENTS}

1. DIII-D NATIONAL PROGRAM OVERVIEW............................................................. 1-1

2. DIVERTOR AND BOUNDARY RESEARCH PROGRAM ..................................... 2-1

2.1. Detached and Radiative Divertor Experiments................................................... 2-1

2.2. H-mode Density Limit Experiments ............................................................... 2-4

2.3. Divertor Impurity Enrichment Experiments ....................................................... 2-6

2.4. Edge Localized Mode Studies ......................................................................... 2-8

2.5. Divertor Erosion ............................................................................................... 2-10

3. ADVANCED TOKAMAK RESEARCH .......................................................... 3-1

3.1. High Performance Discharges with Negative Central Magnetic Shear ................. 3-1

3.2. High Performance Transport Barriers with ExB Electric Field Shear ................... 3-5

3.3. Ion Cyclotron Range of Frequencies Fast Wave Current Drive Experiments ........ $\quad 3-7$

3.4. Electron Cyclotron Range of Frequencies Heating Experiments ........................... $\quad 3-9$

4. TOKAMAK PHYSICS ................................................................................................... 4-1

4.1. H-mode Power Threshold Studies ................................................................... 4-1

4.2. Scaling of Transport with Dimensionless Parameters ........................................... 4-3

4.3. Neoclassical Tearing Modes ............................................................................... $\quad 4-5$

4.4. Disruption Studies ............................................................................................ 4-6

4.5. Noninductive Startup Experiments ................................................................... 4.. 4

4.6. Particle Transport ............................................................................................... $4-9$

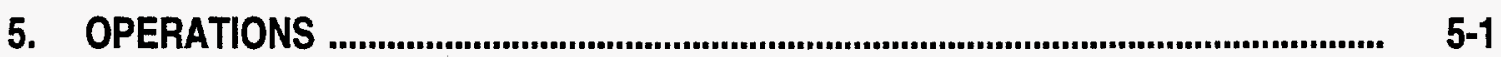

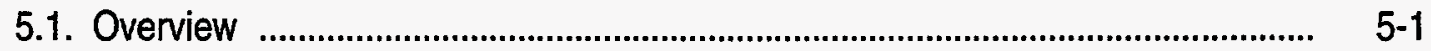

5.2. Tokamak Operations ................................................................................. $5-2$

5.3. Neutral Beam Operations ................................................................................... 5

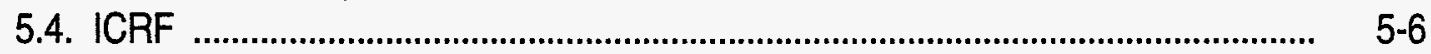

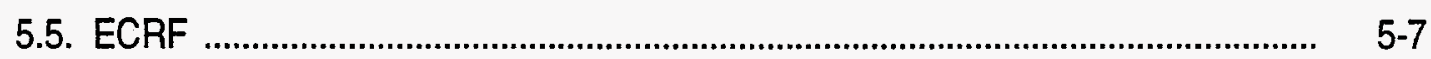

5.6. Computer Systems ....................................................................................... 5

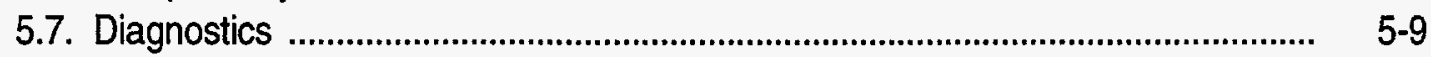

6. PROGRAM DEVELOPMENT …................................................................ 6-1

6.1. ECH Upgrade Project .....................................................................................

6.2. Radiative Divertor Upgrade Project ................................................................... $6-4$

6.3. Vanadium Divertor Structure .............................................................................. $\quad 6-6$

7. SUPPORT SERVICES _.....................................................................................

7.1. Quality Assurance ............................................................................................ 
7.2. Planning and Control

7.3. Environment Safety and Health ........................................................................ $\quad 7-2$

7.4. Visitor and Public Information Program .............................................................. $\quad$ 7-4

8. COLLABORATIVE EFFORTS

8.1. DIII-D Collaboration Program Overview ......................................................... 8-1

8.2. Lawrence Livermore National Laboratory ........................................................... 8 8

8.3. Oak Ridge National Laboratory ........................................................................ 8 8

8.4. University of California, Los Angeles ............................................................. $8-5$

8.5. University of California, San Diego ....................................................................... 8-6

8.6. International Cooperation .............................................................................. $8-7$

9. FY96 PUBLICATIONS _....................................................................................... 9-1

\section{TABLES}

1-1. DIII-D program collaborators ....................................................................

1-2. DIII-D scientific objectives ............................................................................

1-3. DIII-D program 1996 key accomplishments ........................................................ 1-4

1-4. Maximum DIII-D parameters achieved to date 4-1-97 ……................................. 1-8

2.3-1. Divertor enrichment results ............................................................................. $2-7$

5-1. DIII-D system specifications ............................................................................ $5-1$

5.7-1. Diagnostic changes during the 1996-1997 winter vent ........................................ 5-10

5.7-2. Plasma diagnostics at the end of FY96 .......................................................... $5-11$

\section{FIGURES}

1-1. Color enhanced contour plot of measured fluctuation spectrum evolution................. $\quad 1-5$

1-2. Density $1.5 \times$ Greenwald "limit" achieved with H-mode confinement ...................... 1-6

1-3. Upper, lower, and dual divertors with plasma shapes .......................................... $\quad 1-7$

2.1-1. The parallel heat flux profile in the divertor plasma ................................................ $2-2$

2.1-2. Spectroscopically determined radiation fractions ................................................. $2-3$

2.2-1. Data from an $\mathrm{H}$-mode shot .......................................................................... 2-5

2.3-1. Divertor impurity retention experiment .............................................................. 2-7

2.4-1. Scaling of loss normalized to total stored energy .................................................. 2-9

2.5-1. Measured net carbon loss rate at DIII-D ............................................................ 2-10

3.1-1. Experimental profiles of ion temperature ............................................................ $3-2$

3.1-2. Dependence of the beta limit on the current density profile .................................... $3-3$

3.1-3. Fusion gain $Q_{D D}=P_{\text {fusion }} / P_{\text {in }}$ versus plasma current squared .............................. $3-4$ 
3.2-1. $E \times B$ shearing rate greatly exceeds trapped electron ion temperature gradient growth rate

3.3-1. The measured FWCD efficiency and the anomalous neutron rate

3.4-1. Electron temperature before and during $0.5 \mathrm{MW}$ of $\mathrm{ECH}$.

4.1-1. $D_{\alpha}$ shows that very slow transition occurs from about 2525 to $2550 \mathrm{~ms}$

4.2-1. Summary of measured global scalings

4.3-1. Onset of $3 / 2$ and $2 / 1$ tearing in DIII-D fitted to local parameters

4.4-1. Toroidal peaking factor and halo current

5.2-1. Tokamak availability FY93 to present

5.2-2. Downtime for FY96

5.2-3. DIII-D FY96 weekly operations schedule

5.3-1. Neutral beam availability by month

5.3-2. Causes of neutral beam downtime by category

$5-6$

6.1-1. $110 \mathrm{GHz}$ GYCOM gyrotron installed in DIII-D ECH vault ...................................... $6-2$

6.1-2. RF burn pattern $1724 \mathrm{~mm}$ from gyrotron window .................................................

6.1-3. Mirror optical unit ............................................................................................ 6-.

6.2-1. We will employ a phased approach in implementing the RDP ............................... $6-5$

8.2-1. We are developing a physics model of the detached divertor plasma with new 2-D diagnostic data

8.2-2. $M S E E_{r}$ upgrade viewing geometry

8-3

8.4-1. Example of edge density profile evolution through a giant ELM

8-6 


\section{ACRONYMS AND ABBREVIATIONS}

\begin{tabular}{|c|c|c|c|}
\hline ABB & Asea Brown Boveri & LANL & Los Alamos National Laboratory \\
\hline CEDMAT & Combustible, Explosive and Dangerous & LLNL & Lawrence Livermore National Laboratory \\
\hline & Materials & $L-$ mode & lower energy confinement \\
\hline CER & charge exchange recombination & MDS & multichannel divertor visible spectrometer \\
\hline $\mathrm{ClO}$ & Continuous Improvement Opportunities & MHD & magnetohydrodynamic \\
\hline $\mathrm{CPI}$ & formerly known as Varian & MIT & Massachusetts Institute of Technology \\
\hline CPR & cardiopulmonary resuscitation & MOU & mirror optical unit \\
\hline$C X$ & charge exchange & MSE & Motional Stark Effect \\
\hline C-Mod & Alcator, C-Modification & NB & neutral beam \\
\hline DiMES & Divertor Material Evaluation Studies & $\mathrm{NBI}$ & neutral beam injection \\
\hline DOE & Department of Energy & NCS & negative central shear \\
\hline DT & deuterium/tritium & ORNL & Oak Ridge National Laboratory \\
\hline DTS & divertor Thomson scattering & PCS & plasma control system \\
\hline ECE & electron cyclotron emission & PPPL & Princeton Plasma Physics Laboratory \\
\hline $\mathrm{ECH}$ & electron cyclotron heating & QA & Quality Assurance \\
\hline ECRF & electron cyclotron range of frequencies & RDP & Radiative Divertor Project \\
\hline EFIT & equilibrium fitting & RF & radio frequency \\
\hline ELM & edge localized mode & RFI & radio frequency interference \\
\hline FERP & Fusion Energy Research Program & Rl-mode & radiative impurity mode \\
\hline FWCD & fast wave current drive & SCBA & self-contained breathing apparatus \\
\hline GA & General Atomics & SNL & Sandia National Laboratory \\
\hline HWA & Hazardous Word Authorization & SOL & scrapeoff layer \\
\hline$H$-mode & high confinement & SPRED & survey instrument, poor resolution, extended \\
\hline $\mathrm{ICH}$ & ion cyclotron heating & & domain \\
\hline ICRF & ion cyclotron range of frequencies & TFTR & tokamak fusion test reactor \\
\hline ICRH & ion cyclotron resonance heating & TPX & Tokamak Physics Experiment \\
\hline IPMP & Integrated Preventive Maintenance Program & UCLA & University of California, Los Angeles \\
\hline $\mathbb{R}$ & infrared & UCSD & University of California, San Diego \\
\hline IRTV & infrared television & VDE & vertical displacement events \\
\hline ITER & International Thermonuclear Experimental & VUV & vacuum ultra violet \\
\hline & Reactor & VMS & virtual memory system \\
\hline ITG & ion temperature gradient & WNS & weak negative shear \\
\hline JAERI & Japan Atomic Energy Research Institute & & \\
\hline JET & Joint European Torus & & \\
\hline
\end{tabular}




\section{DIII-D NATIONAL PROGRAM OVERVIEW}

The DIII-D National Program is a multi-institutional fusion science research program funded by the U.S. Department of Energy (DOE), and operated by General Atomics (GA). Major research responsibilities are shared by GA, the Lawrence Livermore National Laboratory (LLNL), the Oak Ridge National Laboratory (ORNL), and groups at the University of California campuses at Los Angeles (UCLA) and San Diego (UCSD). In addition, a large number of U.S. and international collaborators contribute focused scientific and operational expertise. A comprehensive list of collaborators is given in Table 1-1.

Table 1-1

DIII-D Program Collaborators

\begin{tabular}{lll}
\hline National Laboratories & Universities & Intemational Laboratories \\
\hline ANL & Cal Tech & Academia Sinica (China) \\
INEL & Columbia U. & Cadarache (France) \\
LANL & Hampton U. & CCFM (Canada) \\
LLNL & Johns Hopkins U. & Culham (England) \\
ORNL & Lehigh & FOM (Netherlands) \\
PNL & MIT & Frascati (Italy) \\
PPPL & Moscow State U. & Ioffe (Russia) \\
SNLA & RPI & IPP (Germany) \\
SNLL & U. Maryland & JAERI (Japan) \\
& U. Texas & JET (EC) \\
Industry Collaborators & U.Washington & KAIST (Korea) \\
CompX & U.Wisconsin & Keldysh Inst. (Russia) \\
CPI (Varian) & UCB & KFA (Germany) \\
GA & UCI & Kurchatov (Russia) \\
Gycom & UCLA & Lausanne (Switzerland) \\
Orincon & UCSD & NIFS (Japan) \\
& & Troitsk (Russia) \\
& & Southwestern Inst. (China) \\
\hline
\end{tabular}

The mission of the DIII-D research program is to advance fusion energy science understanding and predictive capability and to improve and optimize the tokamak concept. A long term goal remains to integrate these products into a demonstration of high confinement, high plasma pressure (plasma $\beta$ ), sustained long pulse operation with fusion power plant relevant heat and particle handling capability. The DIII-D program is a world recognized leader in tokamak 
concept improvement and a major contributor to the physics $R \& D$ needs of the International Thermonuclear Experimental Reactor (ITER). The scientific objectives of the DIII-D program are given in Table 1-2.

Table 1-2

DIII-D Scientific Objectives

- Advance the understanding of fusion plasma physics and guide the physics base of ITER through extensive experimental and theoretical interactions in:

- Magnetohydrodynamic (MHD) stability.

- Transport and turbulence.

- Boundary physics and core-boundary interactions.

- Divertor particle and heat control.

- Wave-particle physics.

- Integrate scientific understanding to demonstrate that a tokamak can be:

- More compact with higher fusion power density, requiring a simultaneous increase in stability limits and reduction in transport.

- A steady-state device, with disruption control, current drive, and handling of the divertor heat and particle loads.

- Demonstrate in an operating device the viability of low activation alloys for fusion systems.

The FY96 DIII-D research program was highly successful, as described in this report. A moderate sized tokamak, DIII-D is a world leader in tokamak innovation with exceptional performance, measured in normalized parameters.

In FY96, the DOE embarked upon restructuring the U.S. fusion program, taking input from expert groups, both from inside and outside of fusion research. An ad hoc Major Facilities Review Panel was convened and charged with providing an optimum plan for gaining maximum scientific benefit from operation of the major U.S. tokamaks (DIII-D, C-Mod, and TFTR) at projected funding levels and to recommend a smooth transition of the U.S. program into FY97 and beyond. The findings of this panel recognized the high quality and quantity of fusion scientific output by these three programs. Regarding the DIII-D program, the panel identified it as uniquely positioned to explore the key fusion physics issues in transport, stability, particle and heat load handling, and radio wave interactions and to provide valuable data for the design of ITER. 
Because of this, and the need to optimize the contributions of the many collaborators, the panel recommended expanded operating time for DIII-D in future years. A synopsis of key FY96 accomplishments in these areas is given in Table 1-3, together with key accomplishments in capability enhancement and collaborative interactions.

Commensurate with the panel's recommendations, the DIII-D program in FY96 continued scientific progress in the critical areas, identified in this annual report, further expanded collaborative programs, notably with the Princeton Plasma Physics Laboratory (PPPL), and moved well ahead on two new upgrade projects which will greatly enhance the output of the program in future years and advance DIII-D toward long term optimized tokamak science goal.

The 1996 experiment program on DIII-D was organized into three primary areas: Divertor and Boundary Physics, Advanced Tokamak Studies, and Tokamak Physics. Each area is a research program in itself. The uniqueness and flexibility of DIII-D together with the mature diagnostic systems allow thorough study of each area, as recognized by the panel recommendation for increased operational time in the future. The resulting DIII-D fusion science accomplishments are described in detail in this report. Here we give an overview of these and highlight a few.

One remarkable highlight is the continued development of the performance results from socalled negative central shear (NCS) discharges (Section 3.1). Transport of the thermal energy stored in a plasma has been a major emphasis of fusion research for nearly five decades. The thermal insulation of the plasma has been too weak. Specialized discharges in DIII-D achieved in FY96 what would have been considered impossible until recently, the reduction of ion thermal transport across the entire plasma volume to values as low as theoretically possible. This neoclassical theory predicted what most expected to be the minimum in transport, a level very acceptable for fusion power plant applications. The experimental result was achieved transiently in DIII-D by merging an internal barrier to thermal transport, due to the negative magnetic shear in the core, with an edge transport barrier generated by shear in the electric field flow shear produced by a transition to the $\mathrm{H}$-mode, long studied in DIII-D. Specialized diagnostics simultaneously measured the sharp reduction of turbulent fluctuations in the plasma, consistent with theoretical models relying upon a sheared electric drift to reduce transport (Section 3.2). Figure 1-1 shows this reduction of turbulence as measured by a scattering diagnostic, operated on DIII-D by the UCLA group.

Another key highlight was motivated by the ITER requirement to operate with good energy confinement at high density, namely density above the so-called Greenwald limit. This was achieved in DIII-D divertor experiments by utilizing simultaneous divertor pumping and central fueling with the injection of frozen deuterium pellets with a pellet gun developed and operated by 
Table 1-3

DII-D Program 1996 Key Accomplishments

- Experimental results provided needed guidance for ITER, insights in fusion plasma science, and progress to a more compact fusion power plant.

- Divertor:

Lower than expected electron temperature indicates role of desirable convection and recombination, leading to new edge physics understanding.

is Carbon identified as primary radiator, even in the presence of other impurities.

is Radiative divertor successfully dispersed $80 \%$ to $90 \%$ of the power before reaching the target plate, reducing localized heat loads.

is Simultaneous operation at high confinement and high density $\left(\tau_{E}=\tau_{E}^{\text {ITER93- }}, n_{e}=1.5 \times n_{\text {Greenwald }}\right)$.

t3 The charge exchange excitation rate of highly stripped neon was measured to greater accuracy than previously known, an important byproduct for basic atomic physics.

- Advanced Tokamak:

Using the NCS scenario, fusion reactivity more than doubled the previous DIII-D record, with equivalent $Q_{D T}=0.32$.

t5 When scaled by size and magnetic field strength, this $Q$ is 2 to 9 times larger in DIII-D than other tokamaks, a result of shaping capability.

is Ion transport at minimum theoretical value in NCS discharges.

* Performed definitive measurements of turbulence suppression accompanying transport barrier formation.

is In an ITER-similar shape, simultaneously achieved good confinement $\left(1.5 \times \mathrm{H}\right.$-mode) and high pressure $\left(\beta_{\mathrm{N}}=4\right)$ at an ITER-relevant safety factor $\left(3<q_{95}<4\right)$.

Key experiments further enhanced confidence in the sheared electric drift model for transport barrier formation.

Successfully electron heating with the new Russian gyrotron, achieving an electron temperature of $10 \mathrm{keV}$.

t5 Achieved a world record $280 \mathrm{kA}$ of core fast wave current drive at a record level of efficiency.

- Tokamak Physics:

is Targeted experiments identified all the signatures of neoclassical island destabilization and the need for methods to reduce this effect.

¿ Disruption-induced halo currents and heat flux were reduced significantly $(\sim 50 \%)$ by pre-emptive impurity pellet injection in ITER critical experiments.

is "Very slow" $\mathrm{L} \rightarrow \mathrm{H}$ transitions identified as examples of theoretically described phase transitions. 


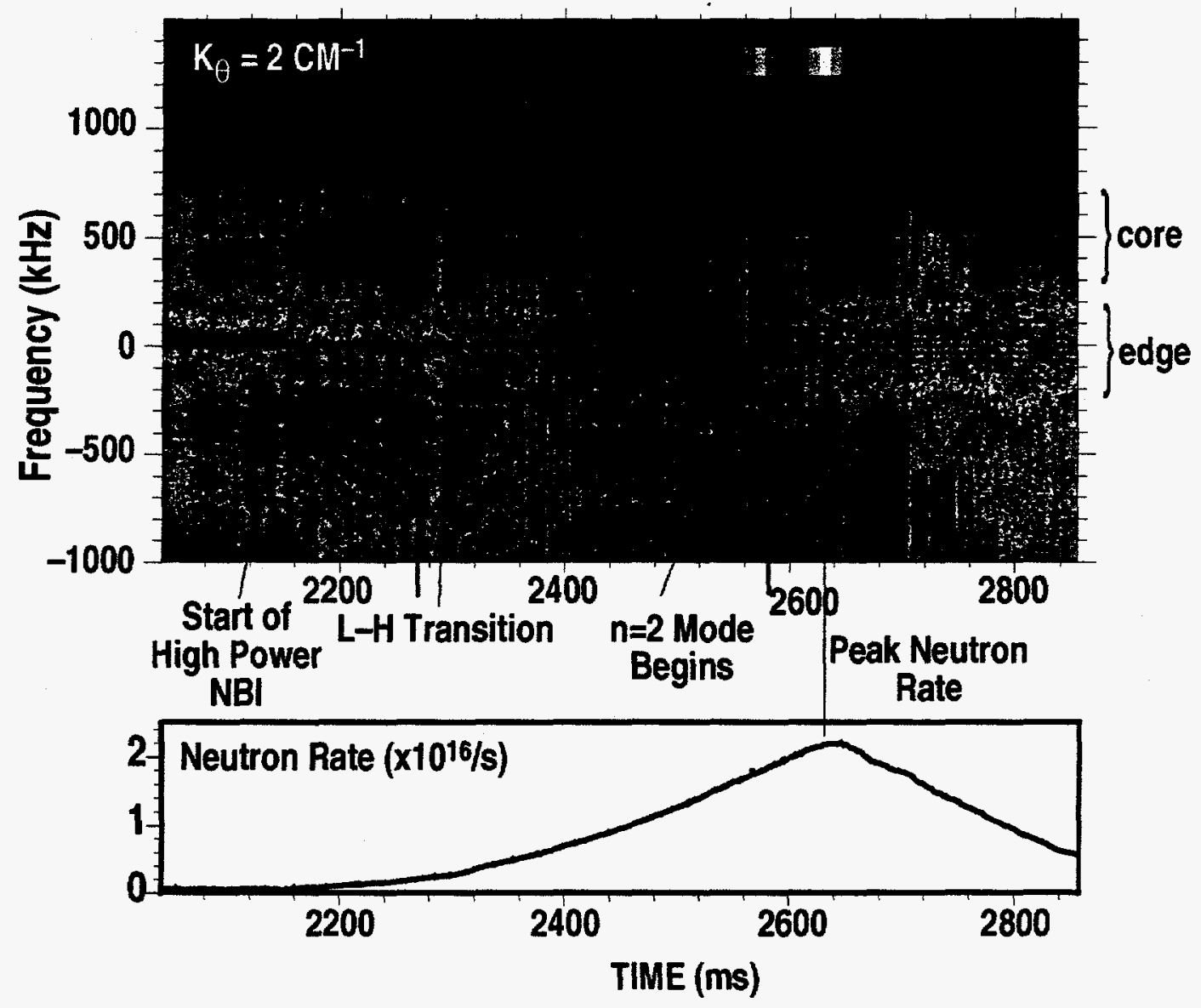

Fig. 1-1. Color enhanced contour plot of measured fluctuation spectrum evolution showing enhanced performance growth (neutron rate) during period of reduced turbulent fluctuations (dark region of contour plot).

ORNL (Section 2.2). Figure 1-2 shows the density and confinement traces from one such discharge. The electron density ratchets up with each injected pellet, eventually triggering a sustained high density in an edge localized mode (ELM)-free $\mathrm{H}$-mode, with energy confinement reaching the $\mathrm{H}$-mode benchmark for ITER $\left(\tau_{\mathrm{E}}^{\text {ITER93-H }}\right)$.

Some of the other key DIII-D accomplishments are: (1) that the duration of the high performance phase in the NCS discharges was extended; (2) in the area of wave heating and current drive, the new Russian gyrotron was used to achieve a new record electron temperature in DIII-D (10 keV), and Fast Wave Current Drive achieved a world record level of $280 \mathrm{kA}$; and (3) in ITER critical experiments, "killer pellets" greatly reduced the deleterious effects of disruptions, and radiative divertor measurements revealed a surprisingly low electron temperature ( 1 to $2 \mathrm{eV}$ ). A summary of the experimental highlights is given in Table 1-3. 

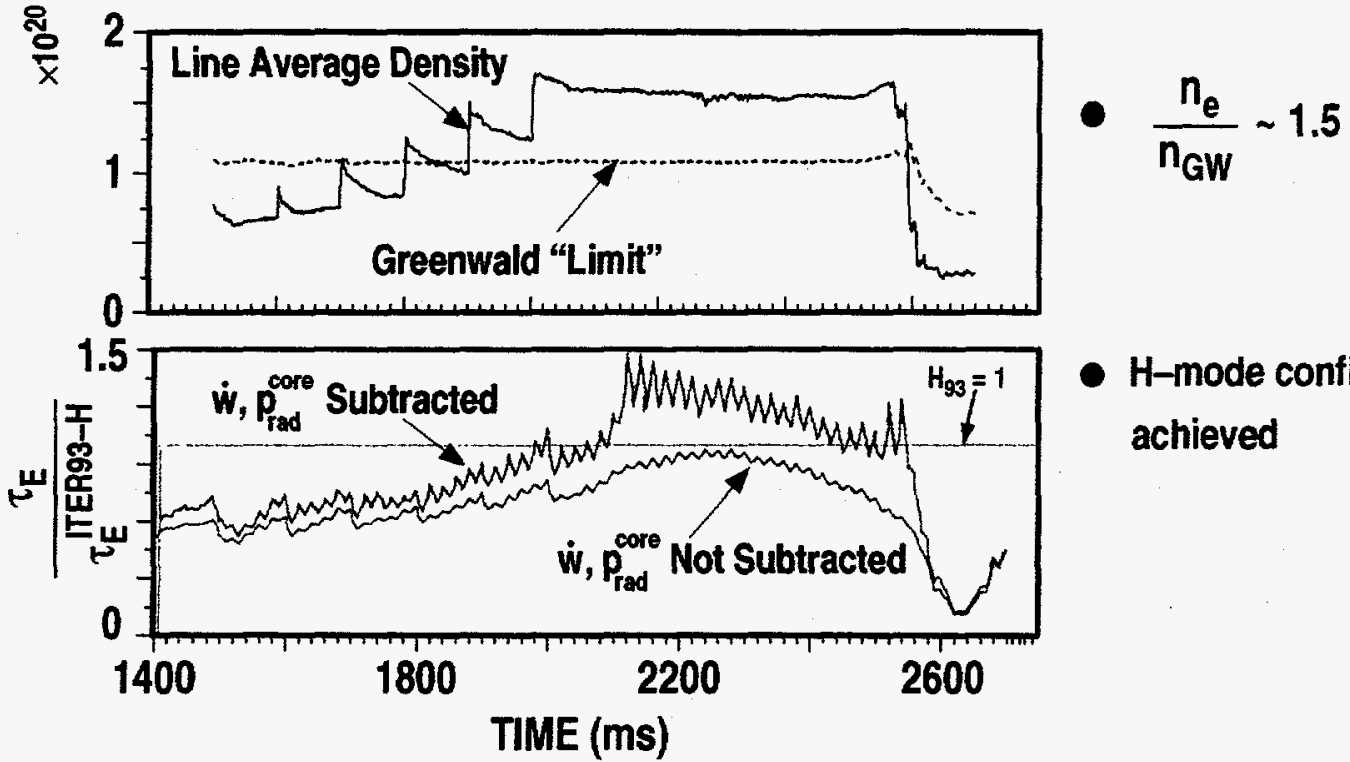

- H-mode confinement achieved

Fig. 1-2. Density $1.5 \times$ Greenwald "limit" achieved with $\mathrm{H}$-mode confinement.

The vigorous international collaboration on DIII-D resulted in unique physics results not available to any one group alone. Using an NCS scenario developed on DIII-D specifically for the Joint European Torus (JET), JET achieved record levels of deuterium-deuterium fusion power in their present containment vessel. A team of DIII-D physicists participated in these important experiments. In another set of experiments, DIII-D and JET operated so-called dimensionally similar discharges, discharges with the same critical physics parameters in appropriately scaled variables yet very different in physical size. Transport analyses of these experiments indicates that such discharges are valid for extrapolation to future machines like ITER. Additionally, over 200 national and international collaborators provide full- and part-time scientific and operational support contributing to the success of DIII-D.

The national DIII-D program also continued to develop new resources and capabilities. New diagnostics and operating modes were conceived and developed. One exciting diagnostic capability will be the ability to measure the electric field interior to the plasma (Sections 5.11 and 8.2). The large electric fields accompanying transport barriers can be directly measured with the Motional Stark Effect (MSE) diagnostic, long used for measuring the internal current profile by measuring the plasma-generated vertical magnetic field. An upgraded MSE system was conceived and designed and will be operational in 1997. Another new capability was the realization of a significant advancement in plasma control by implementing a real-time computer computation of the plasma equilibrium, thereby attaining greater accuracy in the control of the details of the plasma shape. This has been made possible by continued improvement in computational speed. 
Progress was made on two major upgrade projects to enhance the long term DIII-D mission. One upgrade is the installation of a new electron cyclotron heating $(\mathrm{ECH})$ capability. The tokamak physics of ECH was pioneered on DIII-D in the 1980s. GA has become a world leader in developing unique ancillary components for power delivery. The most critical component of the ECH system is the source of high-power millimeter waves, the gyrotron. In 1993, GA took a decisive step to contract with GYCOM, a Russian company, to supply the first $110 \mathrm{GHz}$ gyrotron to DIII-D for this project. This has now proved to be a wise and cost effective decision. The GYCOM gyrotron has been delivered, installed, tested to specification, and used for electron heating in DIII-D, raising the central electron temperature from 3 to $10 \mathrm{keV}$ in the initial experiment (Section 3.3). The next two gyrotrons will be supplied by CPI (formerly Varian) in 1997, and the DIII-D program will make a selection of the vendor to be the supplier for the remainder of the ECH upgrade capability. ECH will give DII-D a source of localized electron heating for definitive transport experiments, for improving performance, and potentially for a source of localized current drive to tailor the steady state current profiles apparently necessary for the advanced confinement and stability regimes. The second upgrade is the Radiative Divertor Project (RDP, described in Section 6.2). Installation of the RDP commenced in FY96 and Phase IA will be completed in mid-FY97. The primary improvements of the RDP, over the first pumped divertor on DIII-D, are a more closed (baffled) divertor for better particle control, and the ability to pump the high-triangularity plasma shapes which can be created by the flexible DIII-D coil system. Figure 1-3 shows
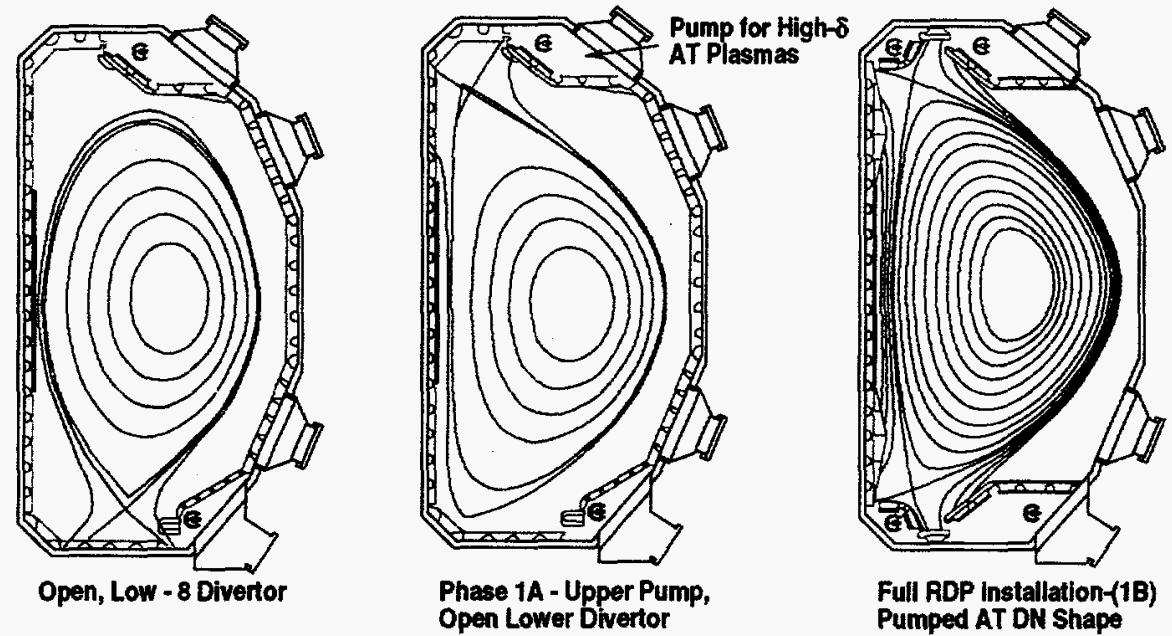

Fig. 1-3. Upper, lower, and dual divertors with plasma shapes.

the existing (lower) open pumped divertor, the present Phase 1A of the RDP (upper), and the future Phase 1B (upper and lower). The triangularity of the shape which strikes the pump is greater for the RDP (as qualitatively revealed by the more interior location of the $x$-points). Experiments on DIII-D have shown high-triangularity to be a key in achieving enhanced 
confinement and performance. The RDP will mate an enhanced divertor capability with the enhanced performance shape.

As described in detail in this report, the DIII-D National Program has again made significant advancements in fusion plasma science, in upgrading the physical capability, and in continually improving procedures and methods. Table 1-4 lists the currently achieved maximum parameters for DIII-D. The parameters in bold were new levels attained in FY96.

Table 1-4

Maximum DII-D Parameters Achieved

To Date 4-1-97, Not Simultaneous

\begin{tabular}{|c|c|c|}
\hline$R$ & $1.67 \mathrm{~m}$ & Nominal \\
\hline$a$ & $0.67 \mathrm{~m}$ & Nominal \\
\hline$\kappa$ & 2.5 & Maximum \\
\hline $\mathrm{B}_{\mathrm{T}}$ & $0.5-2.2 T$ & Range \\
\hline 1 & $3.0 \mathrm{MA}$ & Divertor and limiter \\
\hline$q_{95}$ & 1.75 & Lowest \\
\hline $\mathrm{I} / \mathrm{aB}$ & 3.3 & Maximum \\
\hline$\beta_{\mathrm{T}}(0)$ & $44 \%$ & \\
\hline$<\beta_{T}>$ & $12.5 \%$ & \\
\hline$\beta_{\mathrm{p}}$ & 5.2 & \\
\hline$\varepsilon \beta_{p}$ & 1.8 & \\
\hline $\bar{n}_{e}$ & $\begin{array}{l}3 \times 10^{20} \mathrm{~m}^{-3} \text { (pellet), } \\
1.7 \times 10^{20} \mathrm{~m}^{-3} \text { (H-mode) }\end{array}$ & \\
\hline$T_{e}(0)$ & $10 \mathrm{keV}$ & \\
\hline $\mathrm{T}_{\mathrm{i}}(0)$ & $21 \mathrm{keV}$ & \\
\hline $\mathrm{lbs} / \mathrm{l}$ & $78 \%$ & \\
\hline W & $4.5 \mathrm{MJ}$ & \\
\hline$\tau_{E}$ & $0.4 \mathrm{~s}$ & $\mathrm{P}_{\text {HEAT }}=4.0 \mathrm{MW}$ \\
\hline$n_{D}(0) \tau_{E} T_{D}(0)$ & $7 \times 10^{20} \mathrm{~m}^{-3} \mathrm{~s} \mathrm{keV}$ & \\
\hline D-D neutron rate & $2.4 \times 10^{16} \mathrm{~s}^{-1}(28 \mathrm{~kW})$ & \\
\hline$Q_{D T}$ (equivalent) & 0.32 & \\
\hline $\mathrm{H}$-mode duration & $10.3 \mathrm{~s}$ & \\
\hline$Z_{\text {eff }}$ & 1.1 & \\
\hline$P_{\mathrm{NBI}}$ & $20 \mathrm{MW}$ for $3.5 \mathrm{~s}$ & \\
\hline$P_{E C H}$ & $1.7 \mathrm{MW}$ for $0.5 \mathrm{~s}(60 \mathrm{GHz})$ & \\
\hline $\mathrm{P}_{\mathrm{ICH}}$ & $4 \mathrm{MW}$ for $2.0 \mathrm{~s}$ & \\
\hline
\end{tabular}




\section{DIVERTOR AND BOUNDARY RESEARCH PROGRAM}

\subsection{DETACHED AND RADIATIVE DIVERTOR EXPERIMENTS}

The divertor research in the past year has emphasized investigation of the processes that allow for strong divertor radiation and reduction of the target heat flux. During the previous year, a general characterization of divertor plasmas in various modes of operation was made with many new and improved divertor diagnostics. Now a more thorough study of specific divertor issues and processes is underway. A primary goal of divertor research is to disperse power uniformly throughout the divertor through radiation. An important process in radiative divertor operation is the transport of energy leaving the main plasma and flowing along field lines to the divertor target. The transport of energy is generally thought to be provided by conduction driven by gradients in the electron temperature. In the conduction regime, however, the total amount of radiation will be limited and it will be confined to a small part of the divertor volume. This is due to the nonlinear dependence of electron thermal conductivity which requires steep temperature gradients at lower temperatures, $T_{e} \leq 15 \mathrm{eV}$, where radiation is most efficient.

To determine if conduction is controlling the parallel energy transport in the divertor, the energy flux profile can be readily determined from measurements (described below) and then compared to energy flux predicted from the measured electron temperature distribution assuming classical parallel thermal conductivity. The parallel energy flux distribution is determined by integrating the measured volumetric radiated power distribution starting at the divertor strike plate and proceeding up the flux tube. The starting point heat flux at the strike plate is measured using the IRTV divertor plate heat flux measurement while the volumetric radiation distribution is measured with a two camera, multichord bolometer system. For standard divertor operation, the divertor electron temperature gradient [measured by divertor Thomson scattering (DTS) and shown in Fig. 2.1-1(a)] is consistent with the energy flux carried by conduction. After puffing deuterium gas, the divertor radiation increases sharply dispersing $80 \%$ to $90 \%$ of the power flowing into the divertor before it can reach the target plate [Fig. 2.1-1(b)]. The radiation is also distributed throughout the divertor volume with variations in intensity of no more than a factor of two and the plasma electron temperature (also shown in the figure) drops to $\sim 1 \mathrm{eV}$ throughout most of the divertor. This electron temperature is much too low for conduction to account for the level of energy flux measured in the divertor. 

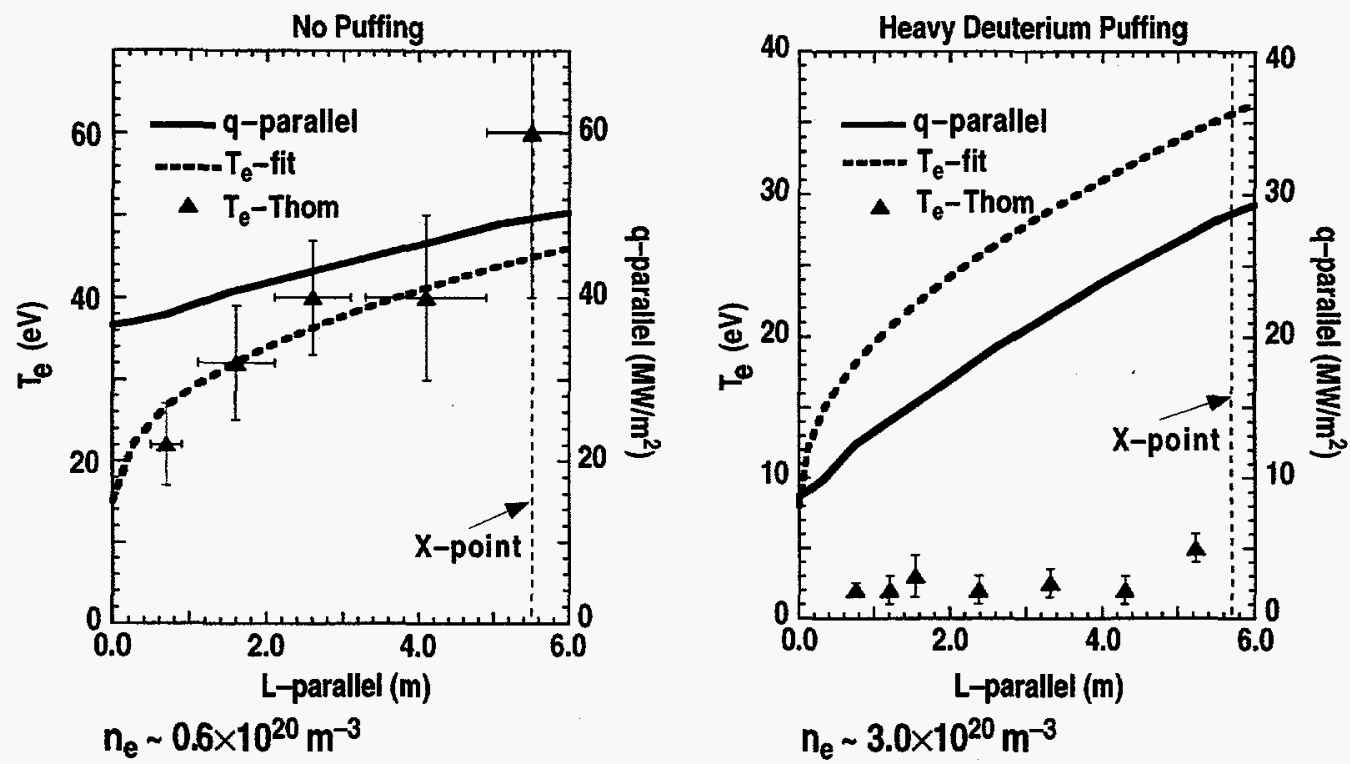

Fig. 2.1-1. The parallel heat flux profile in the divertor plasma for (a) standard operation and (b) radiative divertor operation with deuterium gas puffing. Also shown are the electron temperature profiles calculated to be required for conduction dominated transport ( $\left.T_{e^{-}} \mathrm{fit}\right)$ and the actual $T_{e}$ measurements from DTS.

Plasma flow, or convection of energy, can also be a mechanism for transport of energy. If the assumption is made that the plasma is flowing at the ion sound speed, the observed energy flux can be accounted for. Because of the low electron temperature, $T_{e} \sim 1 \mathrm{eV}$, recycling neutrals can travel upstream to near the $\mathrm{X}$-point before they are ionized. This upstream ionization can provide the source of plasma flow inferred from power balance measurements. Convection is very desirable for radiative divertors because it allows transport of energy through low temperature plasmas where the energy can be more completely and evenly dispersed through radiation and plasma recombination. Operation in this regime may allow the International Thermonuclear Experimental Reactor (ITER) divertor to operate with sufficient radiation spread throughout the divertor to avoid localized heating of the divertor structure.

Also important for understanding divertor operation is determining which species are responsible for the strong radiation. It appears that over the whole divertor, carbon is playing a dominant role in the radiation. An absolutely calibrated vacuum ultra violet survey instrument, poor resolution, extended domain (VUV SPRED) spectrometer views the divertor along a single chord from above. During a sweep of the divertor configuration past this single chord, the line emissivities of CII, CIII, CIV, and Ly- $\beta$ are measured. The measured line emissivities have been used to estimate the contribution of $\mathrm{C}$ and $\mathrm{D}$ to the total radiated power. The measured emissivity of the CIV ion peaks when the viewing chord passes through the vicinity of the $X$-point, whereas 
the Ly- $\beta$ peaks at the strike point, in qualitative agreement with reconstructed visible camera views. The strongest line emission from carbon (CIV $155 \mathrm{~nm}$ ) and deuterium (Ly- $\alpha 121 \mathrm{~nm})$ are not measured by the spectrometer; however, estimates of the local radiation from carbon and deuterium can be made from the measured VUV line emissivities. The reconstructed TV data indicates that the carbon radiation is highly localized. A local electron temperature in the vicinity of the strong carbon radiation is obtained from measured CIV line ratios that are temperature dependent. The obtained temperature $(\sim 7 \mathrm{eV})$ is in reasonable agreement with the temperature measured with DTS in the region of bright visible CIII emission. This temperature is then used, along with a detailed collisional-radiative model of carbon line emission and the measured line emissivities, to estimate the total radiated power for each carbon ionization state and deuterium. The results are shown in Fig. 2.1-2. CIV and D are seen to account for essentially all of the radiated power in the divertor. Near the $\mathrm{X}$-point, CIV heavily dominates; but near the strike point, the CIV and D radiation are approximately equal.

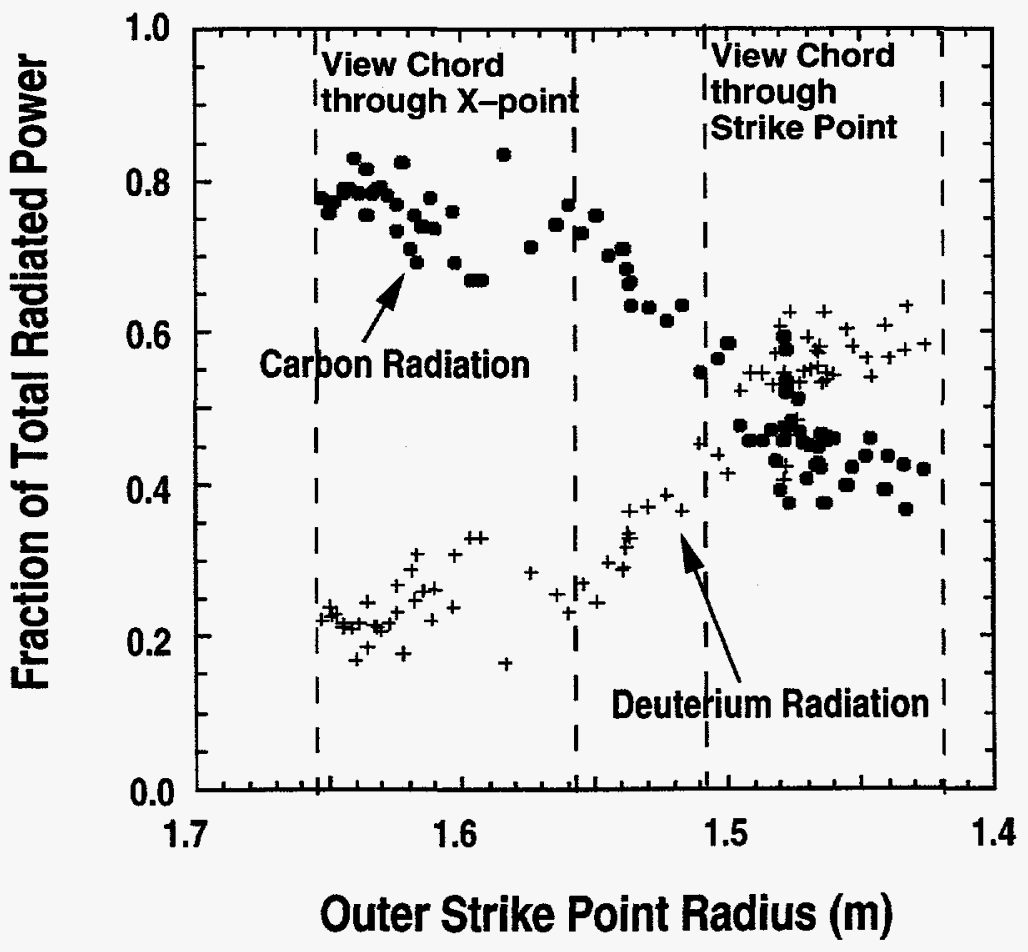

Fig. 21-2. Spectroscopically determined radiation fractions due to carbon and deuterium along the viewchord of the Divertor SPRED spectrometer. The radiation from each species is plotted as a fraction of the total chord integrated radiation along a bolometer chord with a similar view. 


\subsection{H-MODE DENSITY LIMIT EXPERIMENTS}

An overwhelming body of tokamak data support the Greenwald density limit scaling law: $\mathrm{n}_{\mathrm{GW}} \approx \mathrm{I}_{\mathrm{p}} / \pi \mathrm{a}^{2}\left(10^{14} \mathrm{~m}^{-3}\right)$. Several machines [notably, Tokamak Fusion Test Reactor (TFTR), ASDEX-Upgrade, Joint European Tokamak (JET), and DIII-D] under restrictive conditions, have operated at densities above this scaling, albeit at varying degrees of confinement degradation relative to the $\mathrm{H}$-mode. Although the Greenwald limit is not fundamental, it is apparently very difficult to surpass. Motivated by ITER's requirement of $H$-mode operation at $\bar{n}_{e} \geq 1.5 n_{G W}$, we have conducted a series of experiments designed to isolate and independently investigate several physical effects suspected to be directly or indirectly responsible for the density limit. The phenomena studied include: divertor power balance limit, MARFE instability, ballooning mode and $\mathrm{H}-\mathrm{L}$ transition. The experimental knowledge gained was used to simultaneously achieve $\tau_{\mathrm{e}} \approx$ $\tau_{\mathrm{ITER}-93 \mathrm{H}}$ and $\overline{\mathrm{n}}_{\mathrm{e}} \geq 1.5 \mathrm{n}_{\mathrm{GW}}$.

Normally in DIII-D, with either gas or pellet fueling, depending on divertor geometry and heating power, a density limit in the range 0.7 to $1.1 \mathrm{n}_{\mathrm{GW}}$ is observed. This limit is seen following divertor detachment when the most prominent radiation zone reaches the $\mathrm{X}$-point, and is attributed to the divertor power balance limit. We have bypassed this limit by lowering the divertor density relative to the line average density by simultaneous divertor pumping and deuterium pellet injection (pellet diameter $=2.7 \mathrm{~mm}, \delta \mathrm{N} / \mathrm{N} \approx 3 \times 30 \%$ ).

The MARFE condensation instability is a density dependent phenomenon which is normally incompatible with the $\mathrm{H}$-mode confinement. Our data shows conclusively that the Greenwald limit is not explained by this instability. In low powered, high safety factor (q) plasmas, MARFEs were observed at densities below $\mathrm{n}_{\mathrm{GW}}$, whereas at low $\mathrm{q}$ and at a similar power no MARFE activity was observed. In the latter case, the line average density was increased to the symmetric radiative power balance limit at twice the Greenwald limit. Using the data from the high resolution edge plasma diagnostics, we have found that the onset of the MARFE is in good quantitative agreement with the theoretical marginal stability condition [J. Drake, Phys. Fluids 30, 8 (1987)] for this instability.

Several deleterious effects of pellet fueling were observed which reduced the parameter window for high density plasmas. First, near the $\mathrm{H}$-mode power threshold, pellets caused transient $\mathrm{H}-\mathrm{L}$ transitions, which caused unacceptable particle loss. This problem was avoided by lowering $\mathrm{B}_{\mathrm{T}}$ since the power threshold scales inversely with $\mathrm{B}_{\mathrm{T}}$. Secondly, spontaneous or pellet triggered edge localized modes (ELMs) expelled a large fraction of the plasma density which frequently increased the fueling demand beyond the available injection rate. This problem was 
alleviated by lowering ELM frequency through lowering $\mathrm{P}_{\mathrm{INJ}} / \mathrm{I}_{\mathrm{p}}^{2}$. Finally, pellets invariably triggered low number magnetohydrodynamic (MHD) modes which at times continued to grow and lock long after the pellet density perturbation had decayed away. Analysis shows these plasmas to be stable to classical and neoclassical tearing modes. Additional physics is needed to explain these observations. A phenomena similar to "snakes," observed on JET, is suspected and is the subject of continuing investigation.

In order to demonstrate possibility of $\mathrm{H}$-mode confinement at densities above $\mathrm{n}_{\mathrm{GW}}$, we have developed plasma shots with parameters such that the deleterious effects of pellets, MARFE instability, and the divertor density limit were simultaneously avoided. Figure 2.2-1 shows such a shot where $\mathrm{H}$-mode confinement with line average density regulated at 1.5 times the Greenwald limit was attained.

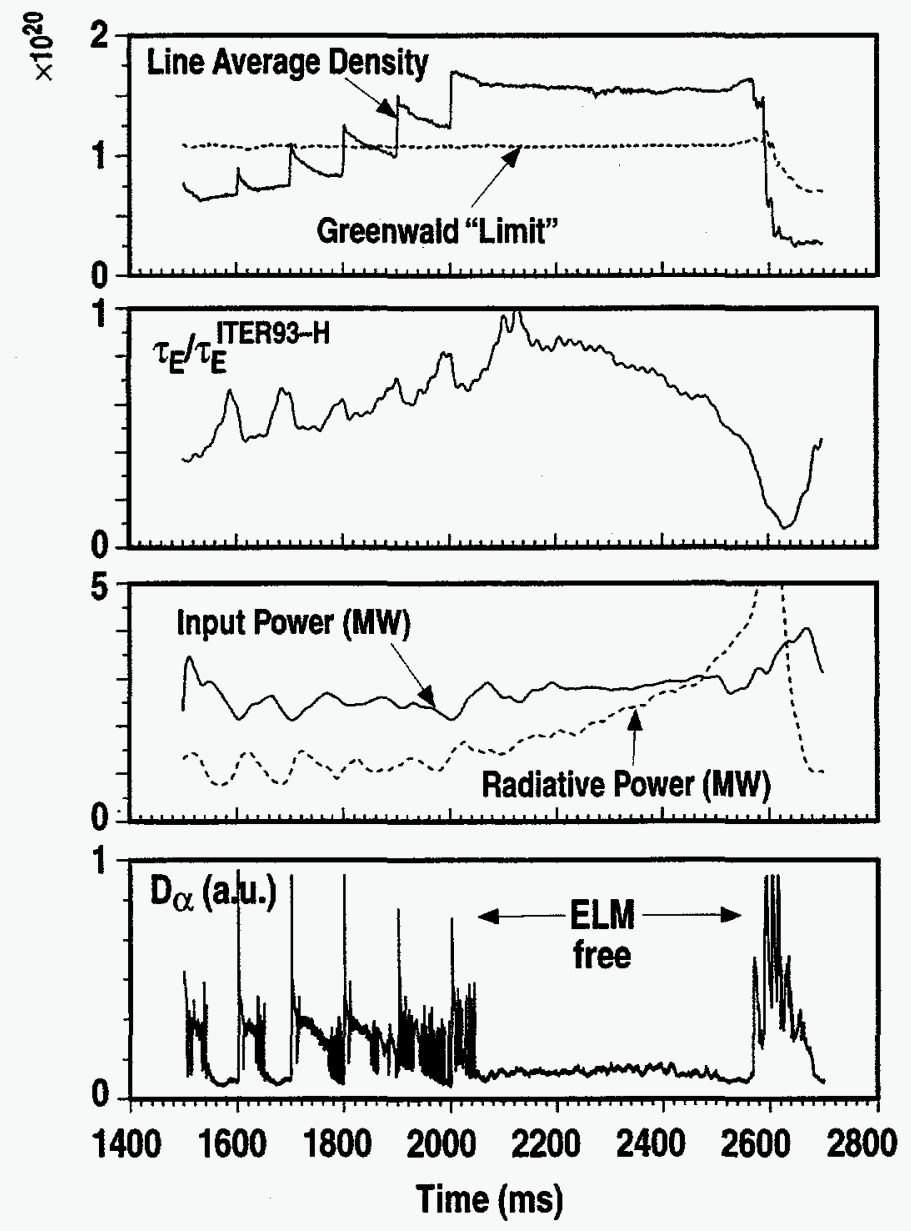

Fig. 2.2-1. Data from an $\mathrm{H}$-mode shot with line average density regulated at 1.5 times Greenwald limit. In this shot, during the density ramp-up global confinement increased to the ITER-93H value. However, ultimately confinement degraded due to the core radiative losses. 


\subsection{DIVERTOR IMPURITY ENRICHMENT EXPERIMENTS}

Divertor impurity enrichment, the ratio of divertor-to-core impurity concentrations, is important because, although a high concentration of radiating impurities is desired in the divertor plasma to disperse exhaust power before it strikes the target, impurities are usually deleterious to the core plasma. Late last fiscal year we made the first direct measurements of exhaust impurity enrichment in a diverted tokamak. (Although divertor enrichment is the quantity of interest, only exhaust gas enrichment can presently be measured quantitatively in DII-D.) This year we ran four days of divertor impurity enrichment experiments and verified and improved the 1995 measurements and extended them from neon as the impurity to argon and helium as well. We showed that "puff and pump" - fueling at the top of the plasma while exhausting an equal amount of gas via the divertor pump at the bottom - increased the exhaust enrichment of trace impurities, with the largest effect observed for argon. It was established spectroscopically that argon was also enriched in the divertor plasma, as desired. At larger neon and argon concentrations that radiated appreciable additional power, we discovered that the majority of the divertor radiation still came from intrinsic carbon. In a noteworthy contribution to atomic physics, the $\mathrm{Ne}^{+10}$ charge exchange (CX) excitation rate was measured in a dedicated experiment and found to be approximately 1.6 times greater than the commonly used theoretical value.

Theoretically, plasma flow toward the divertor is rather slow in most diverted scrapeoff layers (SOLs) so, therefore, does not overcome the upstream thermal force diffusion of $Z>1$ impurities. Impurity entrainment becomes large only in the proximity of the divertor, where ions are neutralized by the target and recycled many times. Gas fueling in the divertor makes only a small perturbation on the large natural recycle flow. However, gas fueling upstream of the divertor can collisionally entrain impurity ions all along the SOL and facilitate divertor impurity enrichment. This principle is illustrated conceptually in Fig. 2.3-1, which shows the concept of our impurity enrichment experiments. Deuterium was puffed near the top of the plasma for the SOL flow cases or under the $\mathrm{X}$-point for the reference divertor fueling cases. In all cases, gas was exhausted at the injection rate via the divertor pump to maintain a steady state. For any given comparison, both plasmas had nearly the same density and temperature profiles, ELM frequency, and exhaust plenum gas pressure. The selected impurity was puffed under the $\mathrm{X}$-point. Full radial density profiles of $\mathrm{He}^{+2}$, (doubly ionized helium) $\mathrm{Ne}^{+10}$, and $\mathrm{Ar}^{+16}$ ions in the core were measured by absolutely calibrated charge exchange recombination (CER) spectroscopy. Impurity partial pressures in the exhaust were measured directly by visible line intensities in a modified Penning gauge. Results for trace impurity levels are summarized in Table 2.3-1. Enrichment increases with atomic number or mass among the noble gases tested. Enrichment also increases with upstream versus 

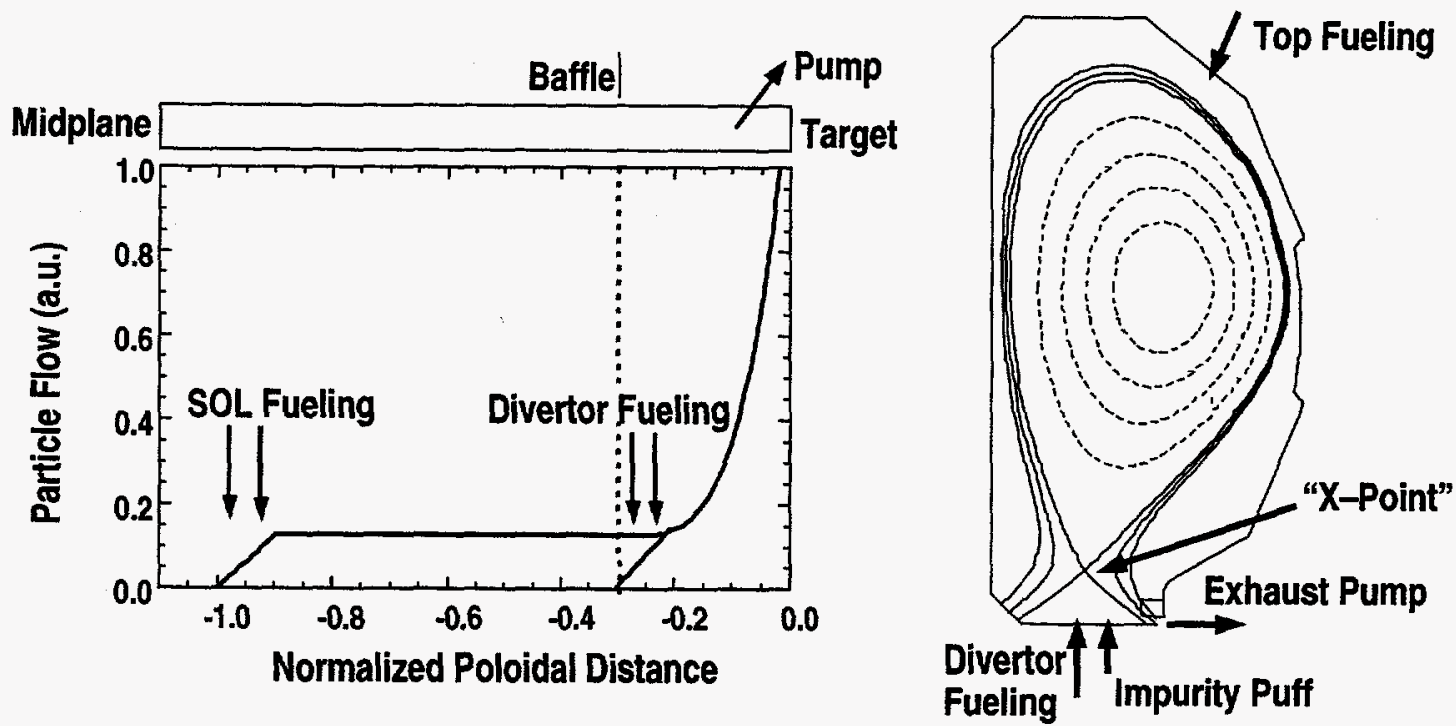

Fig. 2.3-1. Divertor impurity retention experiment. Left: Illustrating how upstream fueling produces particle flow along the SOL that might entrain impurities, whereas divertor fueling does not. Right: Core and exhaust impurity concentrations were measured during deuterium fueling from either a top (upstream) or a divertor location. Divertor pumpout was matched to fueling in all cases.

divertor fueling and with the fueling rate. The ASDEX-U team has published results indicating that the magnitude of divertor recycling, but not SOL flow, affects impurity enrichment in their divertor. This difference remains to be resolved, but it might depend on differences in divertor geometry between the two experiments.

The argon CX excitation rate is still not known accurately, and we give the argon enrichments relative to the divertor low-fueling-rate case. We plan to measure the argon excitation rate in 1997 using a combination of visible bremsstrahlung and soft x-ray techniques.

Table 2.3-1

Divertor Enrichment Results

(Trace Impurity Levels)

\begin{tabular}{|l|c|c|c|c|}
\hline \multicolumn{1}{|c|}{ Fueling Rate } & \multicolumn{2}{|c|}{$20\left(\mathrm{~Pa} \cdot \mathrm{m}^{3} / \mathrm{s}\right)$} & \multicolumn{2}{c|}{$10\left(\mathrm{~Pa} \cdot \mathrm{m}^{3} / \mathrm{s}\right)$} \\
\hline \multicolumn{1}{|c|}{ Fueling Location } & Top & Divertor & Top & Divertor \\
\hline Helium Enrichment & 1.1 & 0.9 & Not Done & Not Done \\
Neon Enrichment & 1.4 & 1.0 & 12 & 1.0 \\
Argon Enrichment (Relative) & 6.9 & 22 & 1.7 & 1.0 \\
\hline
\end{tabular}




\subsection{EDGE LOCALIZED MODE STUDIES}

Edge localized mode (ELM) studies on DIII-D in 1996 concentrated on questions important to the ITER project. Two principle issues were addressed: the scaling of large infrequent (Type I) ELM energy loss and divertor effects to ITER, and the prospects and desirability of operating with low energy loss small and frequent (Type III) ELMs in ITER. Experiments were carried out in ITER shape discharges with varying q (including ITER q values), neutral beam heating power, and L-mode density.

The amount of energy lost from the plasma core during Type I ELMs was determined by calculating the stored energy obtained with the equilibrium reconstruction code [equilibrium fitting (EFIT)] as a function of time over the ELM cycle. In order to accurately follow the fast changes in stored energy at ELMs, data from fast magnetic probe measurements were included in the equilibrium computation. Data from DIII-D was combined with data from the ASDEX-U and JET tokamaks to produce the scaling $\Delta \mathrm{W}_{\mathrm{ELM}} / \mathrm{W}_{\text {TOTAL }} \propto(\mathrm{P} / \mathrm{S})^{-0.4} \mathrm{~B}^{-0.3}$ where $\mathrm{P}$ is the total input power and $S$ is the plasma surface area as shown in Fig. 2.4-1. This scaling predicts an energy loss of $26 \mathrm{MJ}$ per Type I ELM in ITER which is near the limit of what is tolerable in the ITER divertor design assuming a factor of two spreading of the ELM heat flux compared to the steady state heat flux footprint. Taking the ELM power loss as the product of ELM frequency and the energy loss per ELM, we find that approximately $30 \%$ of the input power is lost in Type I ELMs for ITER shape discharges.

The fraction and distribution of the ELM heat pulse which arrives in the divertor was determined from IR camera and bolometer measurements. We measured for ITER shape discharges that between $75 \%$ and $100 \%$ of the energy lost from the core during an ELM arrives in the divertor region with about twice as much going to the inboard relative to the outboard divertor strike point. The ELM energy loss is deposited in the divertor on the time scale of 1 to $2 \mathrm{~ms}$. Less than about 5\% of the ELM energy loss is radiated, probably because of the high power levels associated with a loss of so much energy on this short time scale.

Type III ELMs have the desirable feature that the energy loss per ELM is a factor of 5 to 10 times less than for Type I ELMs. Type III ELMs would thus be desirable for ITER if they were compatible with other aspects of ITER operation.

Two distinct classes of Type III ELMs were identified in this year's operation. The low temperature class, which has been studied extensively on ASDEX-U, occurs below a critical edge 


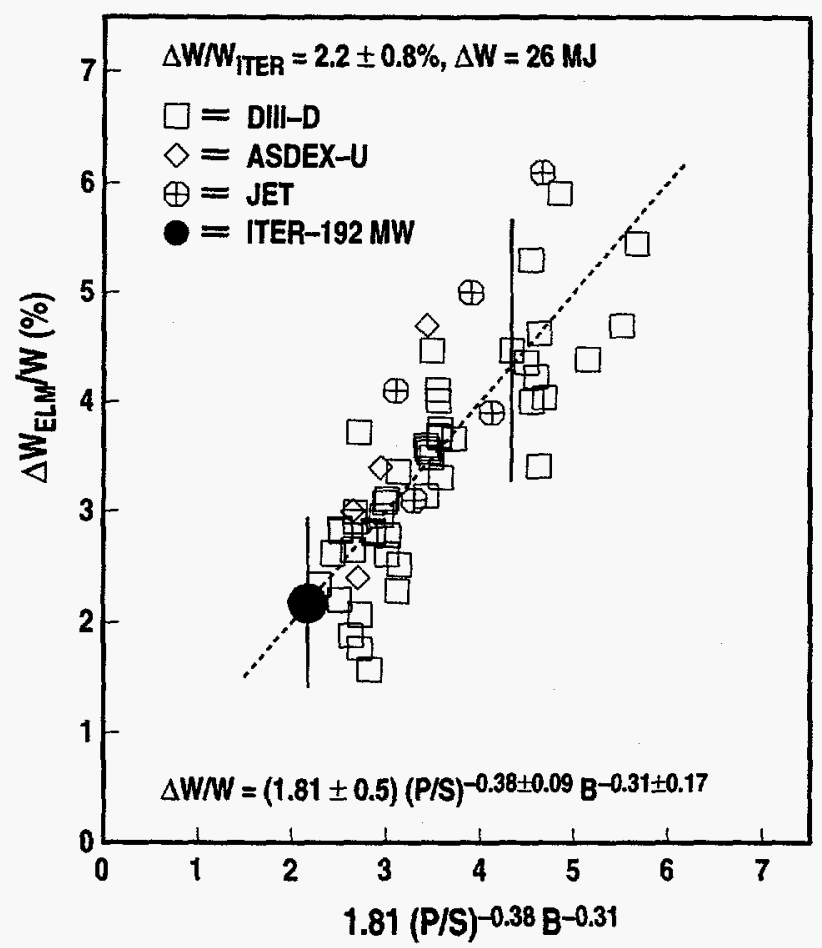

Fig. 2.4-1. Scaling of loss normalized to total stored energy for Type I ELMs and projection to ITER.

temperature. It appears to be possible to achieve high energy confinement with low temperature Type III ELMs if the density is sufficiently high. An understanding of the scaling of the critical temperature for this ELM type will be necessary to determine if they will occur in ITER. A second class of Type III ELMs was identified at low density. In terms of global parameters, low density Type III ELMs disappear above a critical input power which scales as $\mathrm{I}_{\mathrm{p}}^{2.4} / \mathrm{n}_{\mathrm{e}}^{2}$. In terms of local quantities, these ELMs do not exist above a critical edge pressure gradient which scales as $\mathrm{I}_{\mathrm{p}}^{2}$. The density dependence in the global scaling may be tied to the rate at which neutrals fuel the edge pressure gradient. Because of this limitation on edge pressure gradient, the $\mathrm{H}$-mode pedestal and energy confinement is reduced in discharges with low density Type III ELMs. Typically, the energy confinement time, $\tau_{E}$, falls in the range $0.6<\tau_{E} / \tau_{\text {ITER-93H }}<0.9$, where $\tau_{\text {ITER-93H }}$ is the $\mathrm{H}$-mode energy confinement time based upon the scaling developed by the ITER group. This degradation in energy confinement with low-density Type III ELMs may represent a problem for ITER as they can occur at powers near the H-mode threshold power if the density or, perhaps, neutral pressure is low enough. More work needs to be done on the scaling of the threshold conditions for low-density Type III ELMs to determine if they will occur for ITER. 


\subsection{DIVERTOR EROSION}

The Divertor Material Evaluation Studies (DiMES) hydraulic mechanism allows insertion and retraction of graphite samples into the divertor floor of DIII-D. Samples were implanted with a Si depth marker in order to measure the net erosion or redeposition of the graphite. Thin (100 nm) metal films of beryllium, vanadium, molybdenum and tungsten were deposited on the samples to study the erosion, transport, and redeposition properties of these trace metals in the carbon plasmafacing environment of DIII-D. In FY96, we continued to perform erosion and redeposition experiments under normal and disruptive operations. Figure 2.5-1 shows that the net loss of the carbon increases with increasing incident heat flux. These results were obtained during ELM-free and

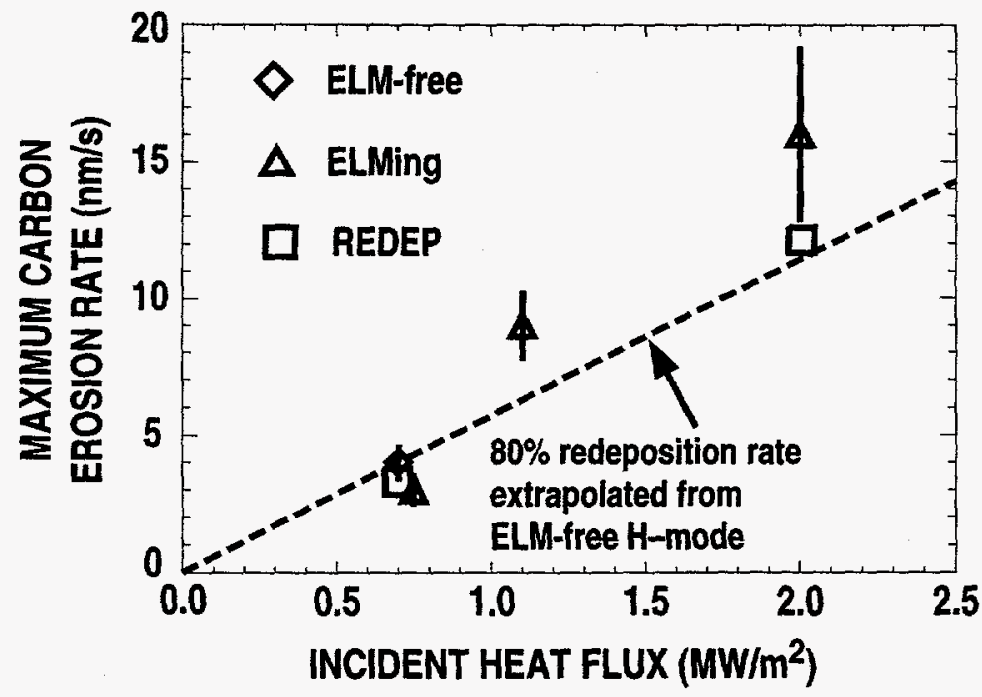

Fig. 2.5-1. Measured net carbon loss rate at DIII-D outer strike point versus incident heat flux measured by infrared themography. $\Delta=$ ELMing $\mathrm{H}$-mode, $\diamond=E L M$-free $\mathrm{H}$-mode. Dashed line is extrapolated net erosion rate from REDEP calculated $80 \%$ redeposition rate of ELM-free $\mathrm{H}$-mode case.

ELMing plasmas using both depth-marking and colorimetry techniques. The REDEP code has calculated a gross erosion of carbon which was five times the measured net erosion rate for the ELMfree case with an incident heat flux of $0.7 \mathrm{MW} / \mathrm{m}^{2}$, corresponding to a redeposition rate of $80 \%$. At higher heat flux, the net erosion is larger than the extrapolated value using this redeposition rate, indicating that the redeposition rate could be decreasing. This is despite the expected increase in local redeposition due to the increased divertor plasma density at the higher heat flux. The effect of ELMs on net carbon erosion appear to be small compared to the erosion from the quiescent plasma. A comparison between the DiMES measured net erosion rates for DIII-D and the predicted carbon 
erosion rates currently used for ITER have shown that the experimental measurements are a factor of 10 higher than the calculation. However, the agreement between the DiMEs results and REDEP is quite good. These differences are under investigation, but are believed to be caused by differences in redeposition efficiency, self-sputtering, and the effect of oblique angles of incidence on sputtering yields. Metallic erosion was examined after the exposure of Be and $\mathrm{W}$ coated samples. Significant amounts of arc tracks are present on tungsten films after plasma exposures and these are contributing to the measured loss rate of the tungsten. The exact cause and phenomenology of this arcing is under investigation. 


\section{ADVANCED TOKAMAK RESEARCH}

\subsection{HIGH PERFORMANCE DISCHARGES WITH NEGATIVE CENTRAL MAGNETIC SHEAR}

The fusion performance of DIII-D has been greatly enhanced this year in plasmas which combine weak or negative central magnetic shear (NCS) with a high confinement (H-mode) edge. An eight-day high performance campaign culminated in the achievement of fusion power and fusion gain more than double the previous record values for DIII-D. A reduction of ion thermal transport to neoclassical levels over the entire plasma volume at high beta was essential to the improved performance. We have made advances in the physics understanding of the mechanisms for transport reduction in these plasmas and their stability limits.

Maximum fusion performance was achieved by the use of simple methods to control both the current density profile and the pressure profile. Low-power neutral beam injection (NBI) is used to heat the electrons during the initial plasma current rise, lowering the plasma resistivity and slowing the penetration of the inductively driven current. This results in a hollow current density profile with a central region of weak or negative magnetic shear. Increasing the beam power leads to the formation of a central region of reduced transport and a strongly peaked pressure profile. Shortly before the discharge would otherwise disrupt because of the strong pressure gradient, an $\mathrm{H}-$ mode transition is triggered which broadens the pressure profile and avoids the disruption.

At peak performance, the internal region of reduced transport has expanded and merged with the $\mathrm{H}$-mode edge transport barrier region, yielding enhanced confinement throughout the entire plasma volume. Far infrared scattering and beam emission spectroscopy measurements show a reduction in density fluctuations over a broad region of the plasma, and transport calculations show that the ion diffusivity is as low as the Chang-Hinton neoclassical value everywhere in the plasma. In these peak performance discharges, the measured ion thermal conductivity falls essentially to zero for all practical purposes; that is, there is no significant energy flux in the ion channel across the plasma. Such small transport rates fall below the measurement threshold. As a verification that the ion transport is consistent with the theoretical neoclassical prediction (also small), we calculate the predicted ion temperature profile assuming neoclassical transport. The agreement is shown in Fig. 3.1-1. As discussed in Section 3.2, these transport observations are consistent with the 
stabilization of microturbulence by $\mathrm{E} \times \mathrm{B}$ flow shear. Weak or negative magnetic shear contributes to stabilization of turbulence; in addition, it provides crucial access to the second stable regime for ballooning modes, allowing the central pressure gradients to increase to the levels necessary for significant $\mathrm{E} \times \mathrm{B}$ flow shear to occur.
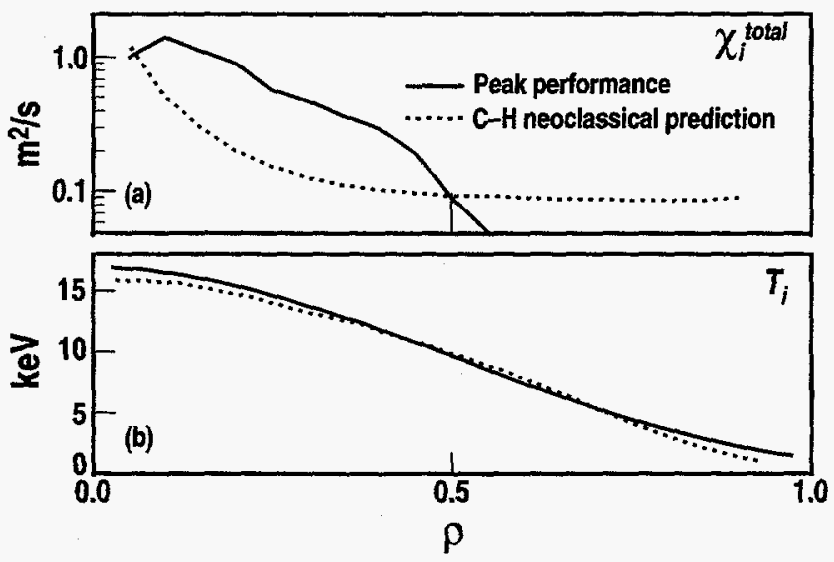

Fig. 3.1-1. Experimental profiles of ion temperature, compared to the predictions of neoclassical theory. This magnitude of transport is so small that the difference in the ion temperature profile is within experimental errors.

The fusion performance of these plasmas is limited by magnetohydrodynamic (MHD) stability. Theoretical calculations predict a strong dependence of the ideal MHD beta limit on both discharge shape and the form of the pressure profile. NCS discharges with a peaked pressure profile and a circular cross section are calculated to have a relatively low beta limit, $\beta_{\mathrm{N}}=$ $\beta /(\mathrm{I} / \mathrm{B}) \approx 2$. Discharge shaping with a peaked pressure profile produces only a modest improvement in the beta limit, as does broadening the pressure profile with a circular cross section. However, with a broad pressure profile and discharge shaping, the calculated beta limit increases to $\beta_{N} \approx 5$ for an elongated, triangular cross-section similar to DIII-D. Consistent with these predictions, NCS discharges in DIII-D with a strongly peaked pressure profile reach a disruptive beta limit at low beta, $\beta_{\mathrm{N}} \leq 2.5$, caused by an internal $\mathrm{n}=1$ mode, while discharges with a broad pressure profile reach significantly higher beta, $\beta_{\mathrm{N}}=4$ to 5 .

In discharges with a broad pressure profile, the performance is limited by instabilities near the edge of the plasma, thought to be driven by the combination of a large pressure gradient and current density in the outer region of the plasma. These instabilities typically have mode numbers $\mathrm{n}>1$ and lead to a soft beta limit, similar to the termination of the good confinement phase in VH-mode discharges. The maximum beta agrees well with the empirical scaling $\beta_{\mathrm{N}} \leq 4 \ell_{\mathrm{i}}$ 
(Fig. 3.1-2). This scaling was found previously for discharges with monotonic q-profiles, which is not surprising since the beta limit for these NCS discharges is caused by instabilities in the outer, positive-shear region of the plasma.

Record values of both fusion power and fusion gain in DIII-D have been achieved in NCS discharges. One such discharge produced $28 \mathrm{~kW}$ of fusion power, while a similar discharge achieved a maximum fusion gain of $\mathrm{QDD}_{\mathrm{D}}=\mathrm{P}_{\text {fusion }} / \mathrm{P}_{\text {beam }}=1.5 \times 10^{-3}$ with $26 \mathrm{~kW}$ of fusion power. The latter case was used as the basis for calculations of the performance of an equivalent discharge with a 50:50 central deuterium-tritium mixture, with all other parameters held constant. Under these conditions, the fusion power is predicted to be about 215 times greater than for the $\mathrm{D}-\mathrm{D}$ mixture, extrapolating to an equivalent fusion gain $\mathrm{QDT}_{\mathrm{DT}} \approx 0.32$ and fusion power $\mathrm{P}_{\mathrm{DT}} \approx$ $6 \mathrm{MW}$.

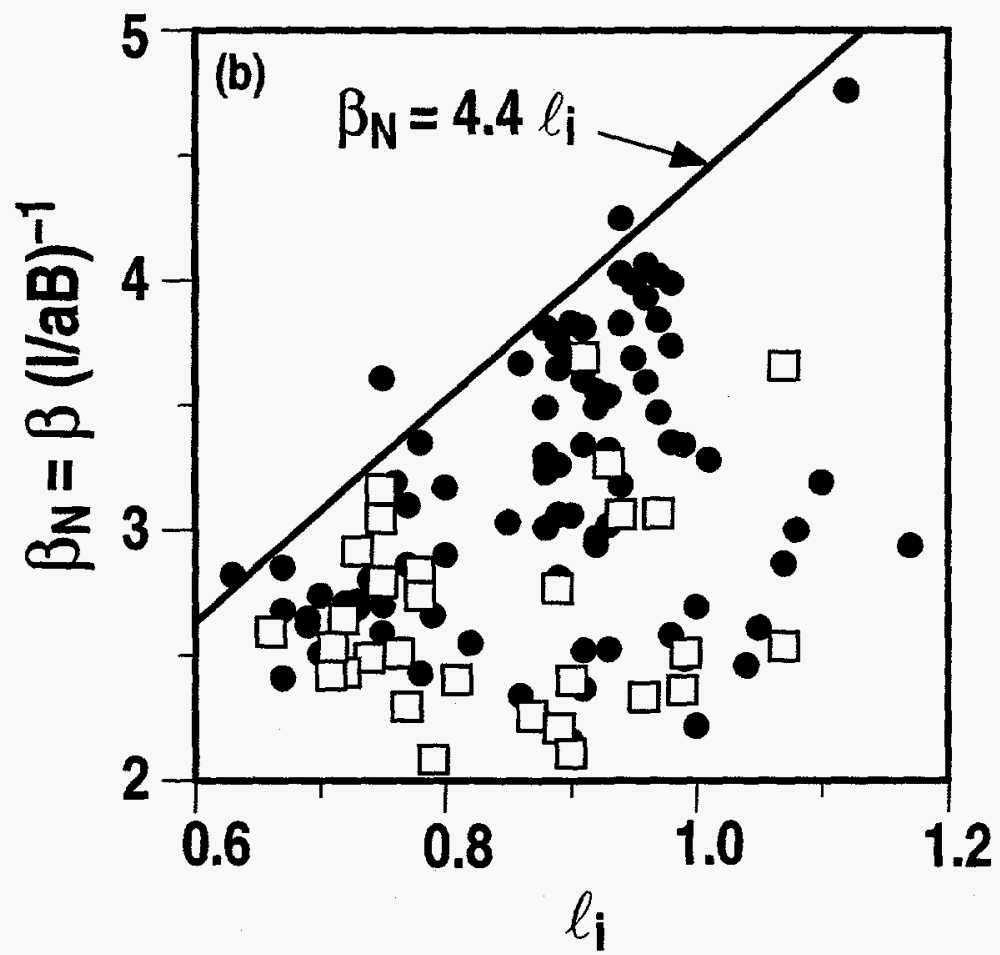

Fig. 3.1-2. Dependence of the beta limit on the current density profile as parameterized by the internal inductance $\ell$, for double-null NCS discharges with $\mathrm{H}$-mode edge and weak or positive shear (circles) or strong negative shear (squares).

High fusion performance in DIII-D depends on several important factors. Since fusion power density is approximately proportional to the square of the plasma pressure, the fusion power 
gain varies roughly as $Q=P_{\text {fusion }} / P_{\text {in }} \sim \beta_{N} \mathrm{HI}^{2} \mathrm{~B}$, where $\mathrm{H}$ is the ratio of energy confinement time to the prediction of ITER-89P L-mode scaling. Fusion power can, therefore, be increased by operating near the stability limit at high normalized beta and with $\mathrm{H}$-mode and, indeed, the maximum QDD was reached at $\beta_{\mathrm{N}}=4$ and $H=4$. Because the fusion power is proportional to the square of the plasma current I (Fig. 3.1-3), substantial improvements in performance can be realized by increasing the plasma current. With other parameters fixed, this means reducing the safety factor; the high QDD discharge in DIII-D was achieved at a moderately low value of $\mathrm{q}_{95}=4.2$. Strong discharge shaping improves the $\beta_{\mathrm{N}}$ limit as discussed above and further improves fusion performance at fixed $\beta_{\mathrm{N}}$ by allowing larger plasma current. The maximum QDD value in DIII-D, when normalized by the field strength and machine size expressed as $B^{2} R^{2}$, is 2 to 9 times larger than values achieved in other tokamaks.

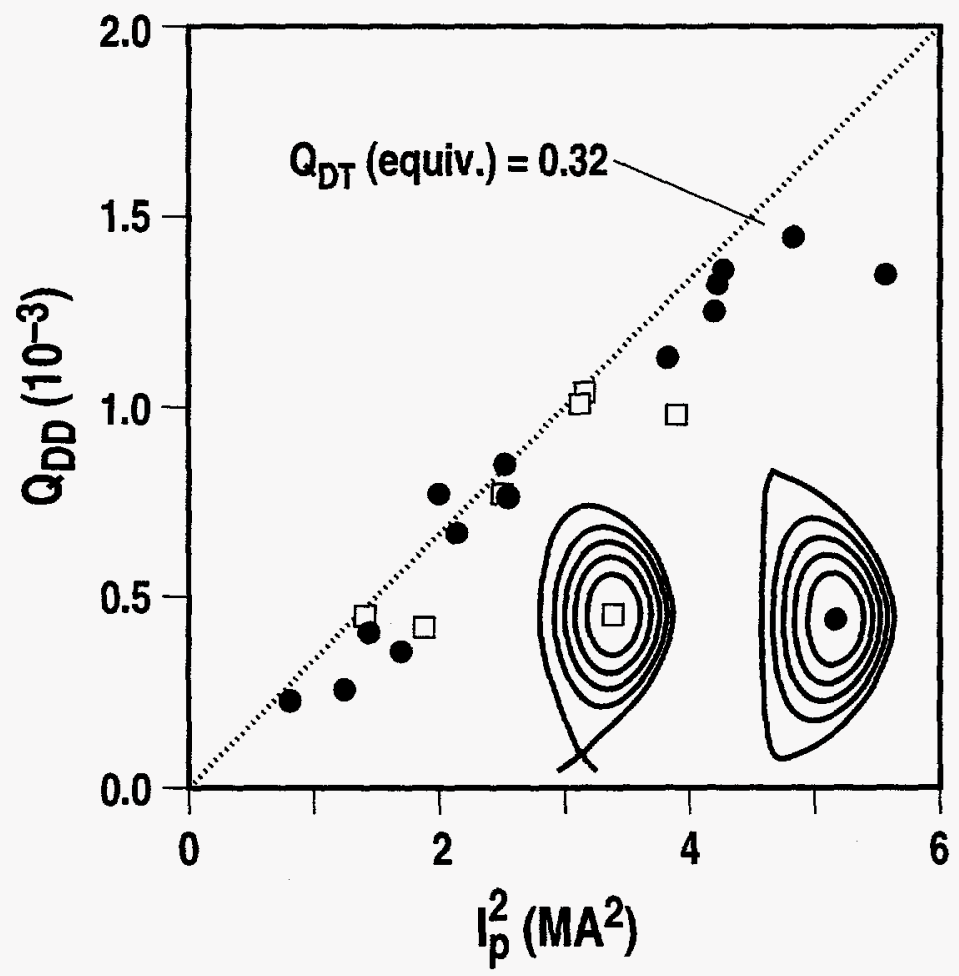

Fig. 3.1-3. Fusion gain $Q_{D D}=P_{\text {fusion }} / P_{\text {in }}$ versus plasma current squared. High triangularity double-null discharges (circles) and low triangularity single-null discharges (squares) have similar dependence on $R$ but the high triangularity cases can reach higher current.

The same approach has also been used to improve the fusion performance of lowtriangularity single-null discharges in DIII-D, with a shape approximating that of Joint European Torus (JET) or International Thermonuclear Experimental Reactor (ITER). Good confinement 
$(H \geq 3)$ and high beta $\left(\beta_{N} \approx 4\right)$ were maintained with safety factors as low as $3<q_{95}<4$, where ITER is expected to operate. The fusion performance of these discharges far exceeded previous values for DIII-D single-null discharges, with central ion temperatures up to $22 \mathrm{keV}$ and fusion gains up to $\mathrm{QDD}_{\mathrm{DD}}=1.0 \times 10^{-3}$. As shown in Fig. 3.1-3, the fusion gain was comparable to that of double-null discharges with the same plasma current; the smaller QDD is a consequence of the smaller current-carrying capacity of the single-null configuration.

The techniques developed in DIII-D were then applied in JET with the collaboration of several DIII-D physicists. As in DIII-D, optimization of the magnetic shear and the pressure profile led to reduced transport and high central pressures without disruption and yielded record values of D-D fusion power for the present JET configuration. Some discharges reached these neutron rates in the presence of small edge localized modes (ELMs) with a period of nearly steady stored energy and fusion rate near the peak; an encouraging result for future steady-state tokamaks.

In summary, a factor of two increase of fusion power in DIII-D has been achieved in the past year through the use of simple profile control tools: early beam heating to modify the current density profile, and a triggered $\mathrm{H}$-mode transition to broaden the pressure profile. These techniques make it possible to combine the favorable properties of weak or NCS in the core with the enhanced edge of the $\mathrm{H}$-mode, yielding enhanced confinement throughout the discharge volume. Electron cyclotron current drive and divertor pumping for edge density control in high triangularity plasmas are new profile control capabilities for DIII-D which should help to improve stability and extend the duration of high performance in future experiments

\subsection{HIGH PERFORMANCE TRANSPORT BARRIERS WITH EXB ELECTRIC FIELD SHEAR}

One of the scientific success stories of fusion research over the past decade is the development of the $\mathrm{E} \times \mathrm{B}$ velocity shear model to explain the formation of transport barriers in magnetic confinement devices. An electric field (E) perpendicular to the magnetic field (B) creates a drift of the charged particles, the so-called $\mathrm{E} \times \mathrm{B}$ velocity. A shear in this velocity means that nearby particles become separated in time because they drift at different speeds. This decorrelation is theoretically predicted to reduce the effect of turbulent transport. This model was originally developed to explain the transport barrier formed at the plasma edge in tokamaks after the L-to- $\mathrm{H}$ transition. Here $\mathrm{L}$ and $\mathrm{H}$ refer to the "low" and "high" confinement modes discovered on the ASDEX tokamak well over 10 years ago. This concept has been recognized to have the universality needed to explain the edge transport barriers seen in limiter and divertor tokamaks, 
stellarators, and mirror machines. More recently, this model has been applied to explain the further confinement improvement from $\mathrm{H}$-mode to VH-mode seen in some tokamaks, where the edge transport barrier extends more deeply into the plasma core. Most recently, this paradigm has been applied to the core transport barriers formed in plasmas with negative or low magnetic shear in the plasma core.

These examples of confinement improvement are of considerable physical interest. It is not often that a system self-organizes to reduce transport when an additional source of free energy is applied to it. In addition to its intrinsic physics interest, the transport decrease that is associated with $E \times B$ velocity shear effects has significant practical consequences for fusion research. For example, the best fusion performance to date in the DIII-D and JT-60U tokamaks has been obtained under conditions where transport reduction through $\mathrm{E} \times \mathrm{B}$ velocity shear decorrelation of turbulence is almost certainly taking place. The performance of these discharges represents a revolutionary step forward in the control of plasma turbulence and transport. In the DIII-D case, for example, the ion thermal transport is at the minimum level set by interparticle collisions over the whole discharge. In other words, at least in the ion channel, it appears that anomalous transport is totally absent.

The fundamental physics involved in transport reduction is the effect of ExB velocity shear on the growth of and radial extent of turbulent eddies in the plasma. Both nonlinear decorrelation and linear stabilization effects have been considered. The basic nonlinear effect is the reduction in radial transport owing to decrease in the radial correlation length and the change in the phase between density, temperature, and potential fluctuations. There are a multitude of linear effects specific to various modes; however, one general feature of linear stabilization is coupling to more stable modes caused by the $\mathrm{E} \times \mathrm{B}$ velocity shear.

The same fundamental transport reduction process can be operational in various portions of the plasma because there are a number of ways to change the radial electric field $E_{r}$. The radial force balance equation

$$
E_{r}=\left(Z_{i} n_{i}\right)^{-1} \nabla P_{i}-v_{\theta i} B_{\phi}+v_{\phi i} B_{\theta}
$$

indicates that there is a connection between $\mathrm{E}_{\mathrm{r}}$ and the cross field heat and particle transport $\left(\nabla \mathrm{P}_{\mathrm{i}}\right)$, cross field angular momentum transport $\left(\mathrm{v}_{\phi \mathrm{i}}\right)$ and poloidal flow $\left(\mathrm{v}_{\theta \mathrm{i}}\right)$. Since sheared $\mathrm{E} \times \mathrm{B}$ flow also affects turbulence and transport, there are several feedback loops whereby $\mathrm{E}_{\mathrm{r}}$ and its shear can change, allowing the plasma access to different confinement regimes. For example, both $v_{\theta i}$ and $\nabla \mathrm{P}_{\mathrm{i}}$ are important in the $\mathrm{H}$-mode edge while $\mathrm{v}_{\phi \mathrm{i}}$ appears to play the major role in $\mathrm{VH}-$ mode. Both 
$\mathrm{v}_{\phi i}$ and $\nabla \mathrm{P}_{\mathrm{i}}$ appear to play a role in the core transport barriers. This multiplicity of feedback loops ultimately provides a number of possibilities for active control of transport. NBI, for example, has been used to alter $v_{\phi i}$.

One of the important themes in this area is the synergistic effects of $E \times B$ velocity shear and magnetic shear. Although the $\mathrm{E} \times \mathrm{B}$ velocity shear appears to have an effect on broader classes of microturbulence, magnetic shear can mitigate some potentially harmful effects of $\mathrm{E} \times \mathrm{B}$ velocity shear and facilitate turbulence stabilization. For example, in the case of core transport barriers, the magnetic shear effects play a role by linearly stabilizing several modes (e.g., sawteeth and ideal ballooning modes) while reducing the growth rates of others, thus allowing the core gradients to steepen. A transport bifurcation, similar to those previously discussed, results and the core transport barrier forms.

An example of the $E \times B$ shear stabilization effect is shown in Fig. 3.2-1. Here, we compare the $\mathrm{E} \times \mathrm{B}$ shearing rate $\omega_{\mathrm{E} \times \mathrm{B}}$ with the linear growth rate of instabilities calculated with the FULL code [Rewoldt, G., et al., Phys. Fluids 30, 897 (1987)]. In this particular case, $\omega_{E \times B}$ exceeds the linear growth rate across the whole discharge. This is connected with the ion thermal diffusivity $\chi_{\mathrm{i}}$ being at or below the Chang-Hinton neoclassical level across essentially the whole discharge, as is also shown in Fig. 3.2-1. Further comparison of data from DIII-D and other machines shows similar spatial and temporal agreement between the theory of $\mathrm{E} \times \mathrm{B}$ shear stabilization and the confinement improvement in the core of discharges with low or negative magnetic shear.

\subsection{ION CYCLOTRON RANGE OF FREQEUENCIES FAST WAVE CURRENT DRIVE EXPERIMENTS}

The physics of fast wave current drive (FWCD) and the interaction of fast waves with energetic beam ions was further advanced this year. In $\mathrm{L}$-mode discharges with negative magnetic shear formed by early injection of neutral beam power, $280 \mathrm{kA}$ of FWCD was achieved with a current drive efficiency of $0.5 \times 10^{19} \mathrm{Am}^{-2} / \mathrm{W}$, both of which are world records. The measured radial profile and magnitude of FWCD are in good agreement with theory based models such as CURRAY and PICES. The FWCD efficiency is found to increase linearly with central electron temperature over a range from 3.7 to $6.4 \mathrm{keV}$; previous FWCD data from DIII-D extends this linear relationship down to about $2 \mathrm{keV}$. Counter FWCD was found to deepen the NCS and delay the onset of sawteeth, compared to co-FWCD, indicating that FWCD can be an effective current profile tool for DIII-D. 


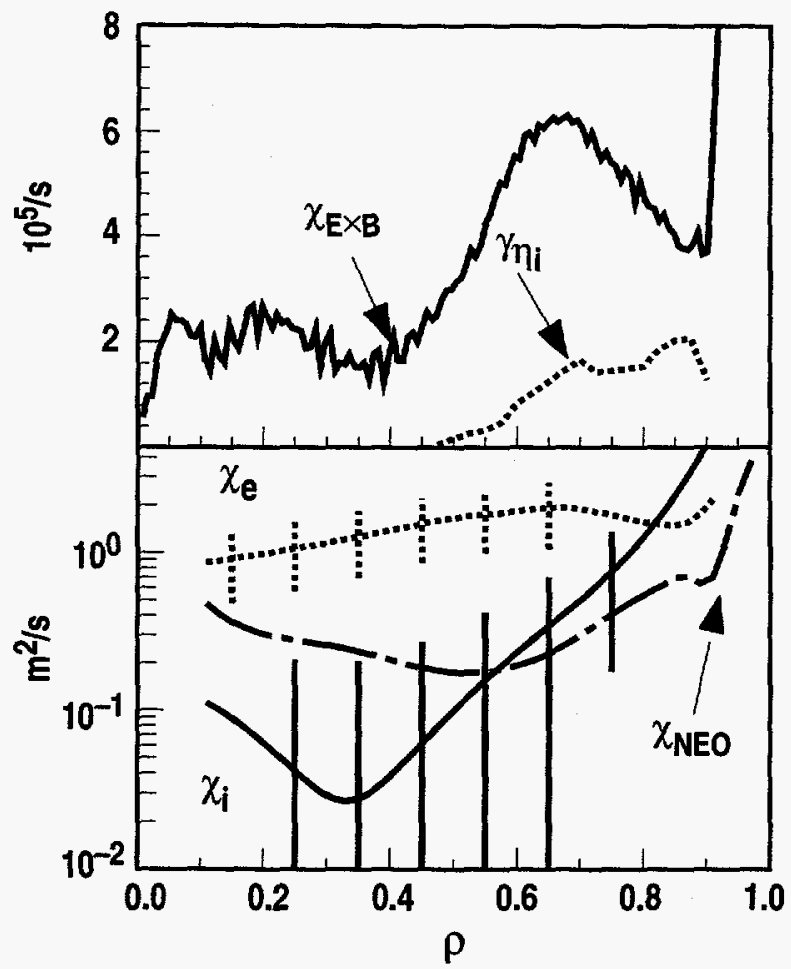

Fig. 3.2-1. EXB shearing rate greatly exceeds trapped electron ion temperature gradient (ITG) growth rate in high performance weak negative shear (WNS) H-mode in DIII-D.

Interaction of the fast waves with energetic beam ions was observed under many conditions on DIII-D and was investigated in some detail. This interaction can be considered as either a benefit for sawtooth stabilization or as a penalty for FWCD. The absorption of fast waves by the beam ions at high harmonics of the deuterium cyclotron frequency manifests itself as an anomalous increase in the central fast-ion stored energy and the neutron rate. A toroidal magnetic field scan found that the anomalous neutron rate peaked at $\mathrm{B}_{\mathrm{T}}=1.9$ and $1.6 \mathrm{~T}$ (see Fig. 3.3-1), where the sixth and seventh $\Omega_{\mathrm{C}, \mathrm{D}}$ resonances pass through the magnetic axis for the principle fast wave frequency of $83 \mathrm{MHz}$. The anomalous fast-ion stored energy mirrors the behavior of the neutrons. Figure 3.3-1 also shows that the FWCD efficiency decreased with lower $\mathrm{B}_{\mathrm{T}}$; the estimated amount of beam ion absorption is of the correct magnitude to explain the reduction in current drive efficiency, although the $\mathrm{B}_{\mathrm{T}}$ dependencies do not agree exactly. The interaction between the fast waves and the beam ions increased with increasing NBI power; however, for magnetic field strengths above $2 \mathrm{~T}$, the effect of beam ion absorption is minimized and the FWCD efficiency is close to the maximum possible value.

FWCD in ELMing $\mathrm{H}$-mode plasmas was also investigated for the first time. The ELMs produced large transients in the antenna loading which produce large reflections of the ion 
cyclotron range of frequencies (ICRF) power. These reflections were diverted from the transmitter by an innovative passive circuit (a combination of a decoupler and hybrid power splitter) which maintained a nearly constant load at the transmitter. For Type-I ("giant") ELMs, efficient fast wave heating and current drive were measured, although the magnitude of the driven current was limited to $\sim 100 \mathrm{kA}$ due to the high H-mode density. For Type-III ("grassy") ELMs, the fast wave heating and current drive were greatly diminished, possibly because of increased edge losses related to the high scrapeoff layer density for this type of $\mathrm{H}$-mode plasma. Future experiments with the upper divertor cryopump may decipher this result.

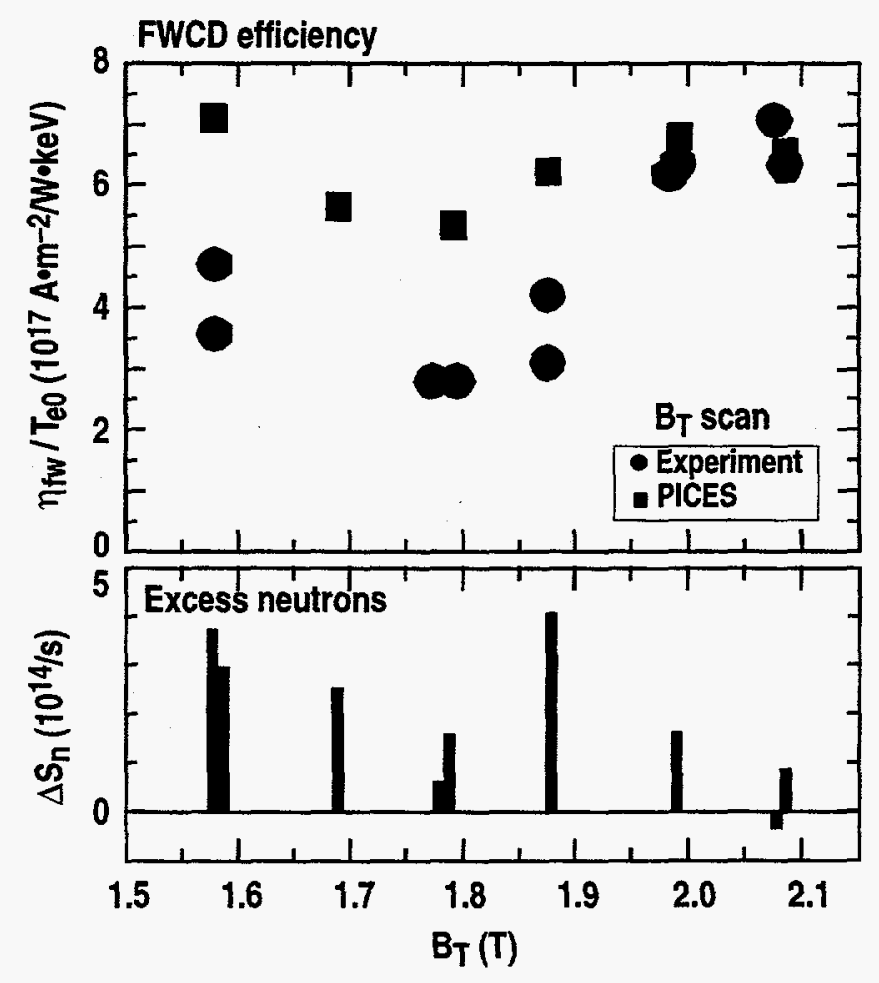

Fig. 3.3-1. The measured FWCD efficiency (normalized to central electron temperature) and the anomalous neutron rate as a function of toroidal magnetic field strength for nearly constant NBI power (3.5 to $4.0 \mathrm{MW}$ ). The theoretical efficiency from PICES is also shown for comparison.

\subsection{ELECTRON CYCLOTRON RANGE OF FREQUENCIES HEATING EXPERIMENTS}

Very high frequency waves with free space wavelength the order of a few millimeters, can be used to heat the electrons by resonating with the electron's gyromotion in the magnetic field of a tokamak. This is referred to as electron cyclotron heating (ECH). ECH requires a high power 
source of millimeter waves generated from the gyrotron tube. This gyrotron tube was developed in the former Soviet Union during the 1970s.

During this year, the first operation of the $110 \mathrm{GHz} \mathrm{ECH}$ system for plasma heating experiments in DIII-D was obtained. Data was taken with on-axis and off-axis aiming of the wave launcher using both steady pulses and modulation of the power at $50 \mathrm{~Hz}$. The GYCOM gyrotron produced reliable pulses up to $500 \mathrm{~ms}$ in duration; pulse extension to $2 \mathrm{~s}$ will be done in FY97 with the vendor's (GYCOM) technical representative at DIII-D. The power diagnostics for the gyrotron are not fully developed at present, but comparisons to fast wave direct electron heating on the same shots implied that approximately $0.5 \mathrm{MW}$ was being delivered to the plasma by the ECH. Low density discharges showed central electron temperatures as high as $10 \mathrm{keV}$, as shown in Fig. 3.4-1. The $\mathrm{OH}$ profile shows the electron temperature prior to application of the $\mathrm{ECH}$ power.

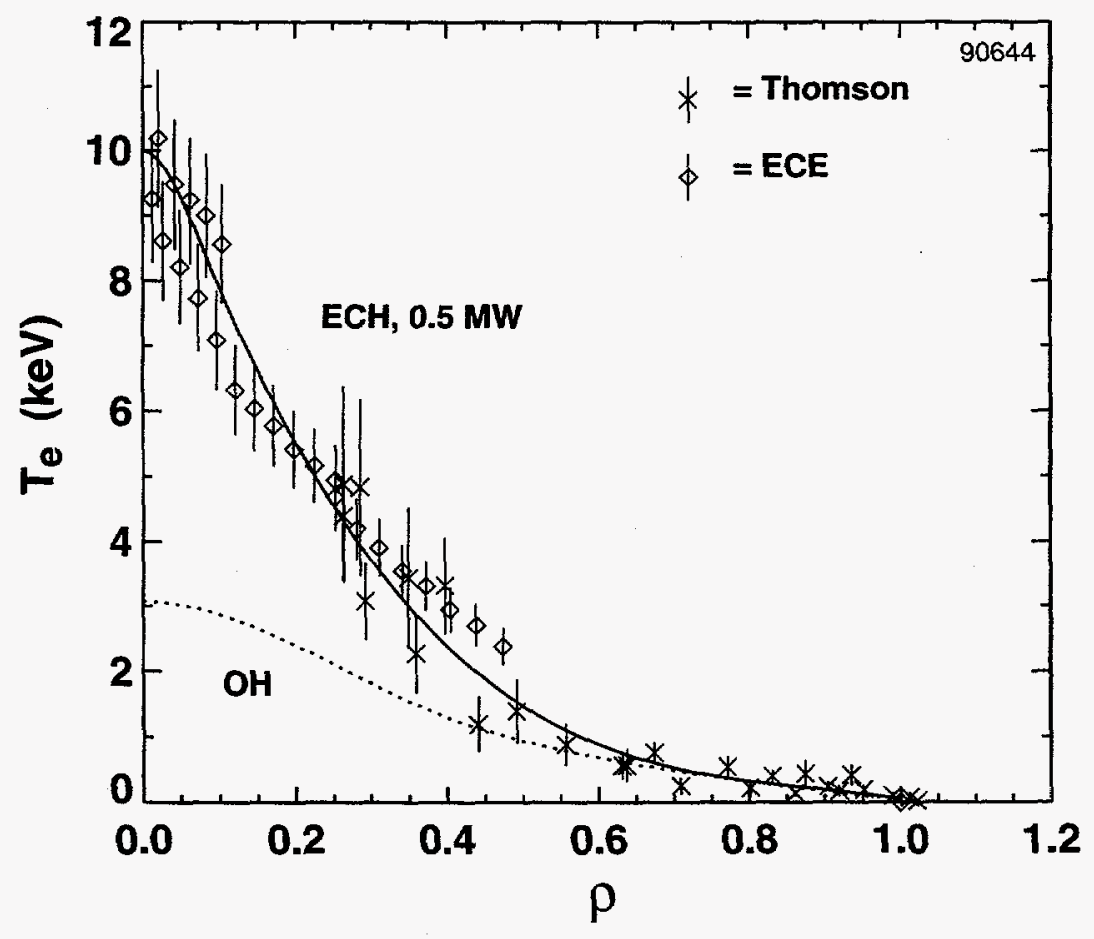

Fig. 3.4-1. Electron temperature before and during $0.5 \mathrm{MW}$ of $\mathrm{ECH}$ as a function of normalized minor radius. The toroidal magnetic field is $1.96 \mathrm{~T}$ and the plasma density is $0.5 \times 10^{19} \mathrm{~m}^{-3}$. 


\section{TOKAMAK PHYSICS}

\subsection{H-MODE POWER THRESHOLD STUDIES}

An active campaign of $\mathrm{H}$-mode experiments and analysis produced several significant results in 1996. (1) A detailed study showed that the "very slow" transitions appear to be examples of "phase transitions" as proposed by Diamond and Carreras. (2) Evidence was obtained that there are hidden variables controlling the $\mathrm{H}$-mode power threshold and that these variables are related to the physics of the scrapeoff layer (SOL) or divertor. If correct, these ideas will ultimately have a profound impact on our understanding of the $\mathrm{H}$-mode power threshold. (3) Substantial progress was made in our characterization of edge profiles and their gradients in lower energy confinement (L-mode) and in $\mathrm{H}$-mode.

"Very slow" $H$-mode transition, in which the characteristic drop of the $\mathrm{D}_{\alpha}$ signal takes 30 to $60 \mathrm{~ms}$, were studied in detail and found to show all of the characteristics associated with more typical "fast" transitions. Of particular interest, the gradients and the radial electric field $\mathrm{E}_{\mathrm{r}}$ in the transport barrier develop gradually during the drop of the $\mathrm{D}_{\alpha}$ signal (Fig. 4.1-1). This phenomenology is consistent with the notion that the $\mathrm{H}$-mode transition is a phase transition and that the very slow transitions occur when the power flow through the plasma edge is very near the "critical point" for producing the transition. Thus, the transition time becomes long and the pressure profile responds self-consistently to the increasing $\mathrm{E}_{\mathrm{T}}$ and the suppression of turbulence drive. As a result, the turbulence suppression is much less dramatic than in fast transitions. The slow $\mathrm{D}_{\alpha}$ drop in these transitions is correlated with the burnout of a MARFE just above the X-point. The MARFE appears to regulate the edge conditions, possibly the temperature, such that the transition phenomenology occur slowly. This observation is strong evidence that divertor and SOL physics can play an important role in the transition to $\mathrm{H}$-mode and may provide hidden variables in influencing the power threshold.

Another SOL variable which may influence the power threshold is the neutral density. An experiment was conducted to examine this issue. The results indicate that it is very difficult to separate the effects of neutrals from the effects of plasma density. Further progress in the study of neutrals will require modeling with neutrals codes. For this purpose, a collaboration was initiated with Ben Carreras and Larry Owens of Oak Ridge National Laboratory who are performing the 
required modeling of DIII-D discharges. Their initial results show clearly that the spatial distribution of the neutral density is very non-uniform with the highest concentrations occurring near the $\mathrm{X}$-point. Thus, any important neutral physics probably occurs in that vicinity.
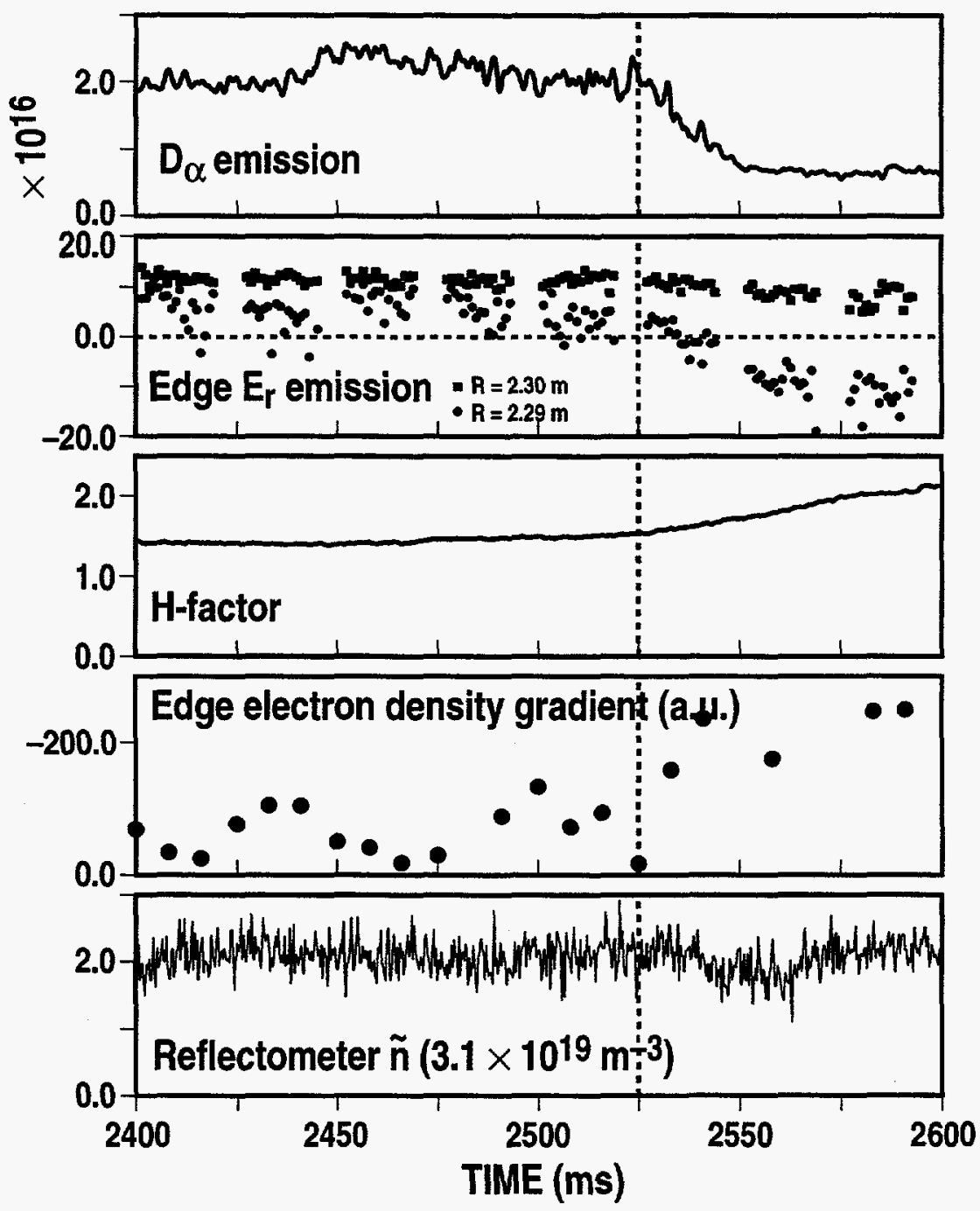

Fig. 4.1-1. $D_{\alpha}$ shows that very slow transition occurs from about 2525 to $2550 \mathrm{~ms}$. During this time, edge $E_{\mathrm{r}}$ gradually becomes more negative, the plasma $\mathrm{H}$-factor gradually improves, and the gradient of electron density in the transport barrier gradually increases. Edge density fluctuations change little.

Another important result of the neutrals experiment was that a sequence of discharges was performed in which the threshold power decreased as the line-averaged density was increased. This trend is opposite to generally observed scalings. Because the primary knobs in the experiment changed conditions in the divertor and SOL, this result points to divertor physics as playing a significant role in the power threshold. Indeed, analysis of divertor data are consistent with the 
notion, suggested by theoretical work of Hinton and Staebler, that the power threshold is decreased when there is a heat sink in the divertor that is near the $\mathrm{X}$-point.

Studies of back (H-L) transitions were initiated. These studies were primarily of edge localized mode (ELM)-free discharges which reverted to L-mode as the heating power was decreased. Significant hysteresis is observed in DII-D. Scaling data for the H-L transition are not available, but the $\mathrm{H}$-mode is often maintained with a power flow through the separatrix which is half of the power required to produce the $\mathrm{L}-\mathrm{H}$ transition. Because the $\mathrm{H}$-mode exhibits a hysteresis cycle, it is of interest to look for the conditions which link the $\mathrm{L}-\mathrm{H}$ and $\mathrm{H}-\mathrm{L}$ transitions. Analysis of local edge conditions shows that the edge electron temperature $T_{e}$ typically shows little hysteresis while the edge density shows considerable hysteresis. That is, in ELM-free discharges, the back transition occurs as the edge $\mathrm{T}_{\mathrm{e}}$ drops to near the value it had prior to the $\mathrm{L}-\mathrm{H}$ transition. This result is consistent with other data suggesting that the condition for having an $\mathrm{H}$-mode is related to edge temperature.

The ability to study local edge parameters was significantly improved with the development of an analysis technique which fits edge profiles with a hyperbolic tangent function. This technique works well for density profiles in L-mode and for density, temperature, and pressure profiles in $\mathrm{H}$-mode. Profiles fit in this way can be parameterized with a small number of parameters, which have strong intuitive appeal. In particular, the height and width of the H-mode pedestal can be readily determined by this process. The hyperbolic tangent fit was used in initial studies of the scaling of the $\mathrm{H}$-mode pedestal relative to plasma parameters. In addition, techniques have been developed to use this fit to study local edge conditions just prior to the $\mathrm{L}-\mathrm{H}$ transition. These tools have been used to develop a database which should yield insights into the important edge parameters controlling the $\mathrm{H}-$-mode transition.

\subsection{SCALING OF TRANSPORT WITH DIMENSIONLESS PARAMETERS}

Significant progress was made this year in determining the scaling of heat transport with dimensionless plasma parameters in both $\mathrm{L}$-mode and $\mathrm{H}$-mode plasmas. The rationale behind adopting this scaling approach as opposed to the traditional scaling with engineering parameters is twofold. First, the dimensionless parameters have physical significance in the theories of heat transport and also known physical limits. For example, in the engineering approach, the scaling with plasma current $I$ is linear, motivating raising current to increase confinement. However, there are limits on the dimensionless parameter $\mathrm{q}$ (roughly $\mathrm{a}^{2} \mathrm{~B} / \mathrm{RI}$ ) which preclude raising current arbitrarily. This limit is easily accounted for in the dimensionless parameter scaling, since $\mathrm{q}$ is used 
directly as the dependent variable. Second, present-day tokamaks can produce plasmas with the dimensionless quantities equal to those projected for a fusion power plant with the exception of a single quantity - the ion gyroradius normalized to the plasma size, $\rho_{*}$. This reduces the confinement projection problem from a multi-variable regression to a one-dimensional scaling.

One proof of the validity of the dimensionless parameter scaling approach is to make plasmas on two different machines with widely different engineering parameters but identical dimensionless parameters. If the normalized power is also identical, then the approach is valid. Experiments of this type have been carried out in collaboration with the JET team. The type of discharge chosen for the match is an ELMing $\mathrm{H}$-mode plasma which is the expected operational scenario for International Thermonuclear Experimental Reactor (ITER). The global parameters on the two machines match to $<5 \%$ as does the normalized power. This provides some confidence in applying this scaling principle to the design of future devices. Local transport comparisons are still in progress, but appear to support the validity of this approach point-by-point spatially in addition to the global results.

These scaling experiments in plasma $\beta$ (plasma pressure scaled to magnetic field pressure) and $v_{*}$ (collisionality) were carried out in both $\mathrm{L}$-mode and $\mathrm{H}$-mode plasmas this year. In L-mode plasmas, the global confinement, $\tau$, has a very weak degradation with $\beta\left(\tau \propto \beta^{-0.1}\right)$ and is virtually independent of collisionality. The local transport analysis agrees with the global results. In $\mathrm{H}$-mode plasmas, the global confinement has a slightly favorable $\beta$ scaling $\left(\tau \propto \beta^{0.1}\right.$ ) and has a noticeable degradation with collisionality $\left(\tau \propto v_{*}^{-3}\right)$. Again the local transport analysis supports the global results. In combination with our previous observation that the electron heat transport is gyro-Bohm like $\left(\tau \propto \rho_{*}^{-3}\right)$, these results are consistent with expectations based on electrostatic drift-wave theories of confinement. These theories predict gyro-Bohm $\rho * s c a l i n g$, weak $\beta$ scaling, and no collisionality scaling. The slight collisionality scaling is consistent with a modest level of drift-wave transport mixed with neoclassical ion transport which has $\tau \propto v_{*}^{-1}$.

The H-mode results were confirmed by experiments on the JET tokamak. The surprising result from these studies is that the "scaling laws" for confinement derived from regression analysis of multi-device databases predict much larger degradation of confinement with $\beta$. For example, the most popular L-mode scaling, ITER-89P, has $\tau \propto \beta^{-0.52}$. The standard H-mode scaling, ITER-93H, has an even more dramatic degradation $\tau \propto \beta^{-1.23}$. The measured global scalings (including the previous $\rho_{*}$ scaling experiments) are summarized in Fig. 4.2-1. At present, the origin of the large difference in $\beta$ scaling from the standard scaling algorithms is not known. 

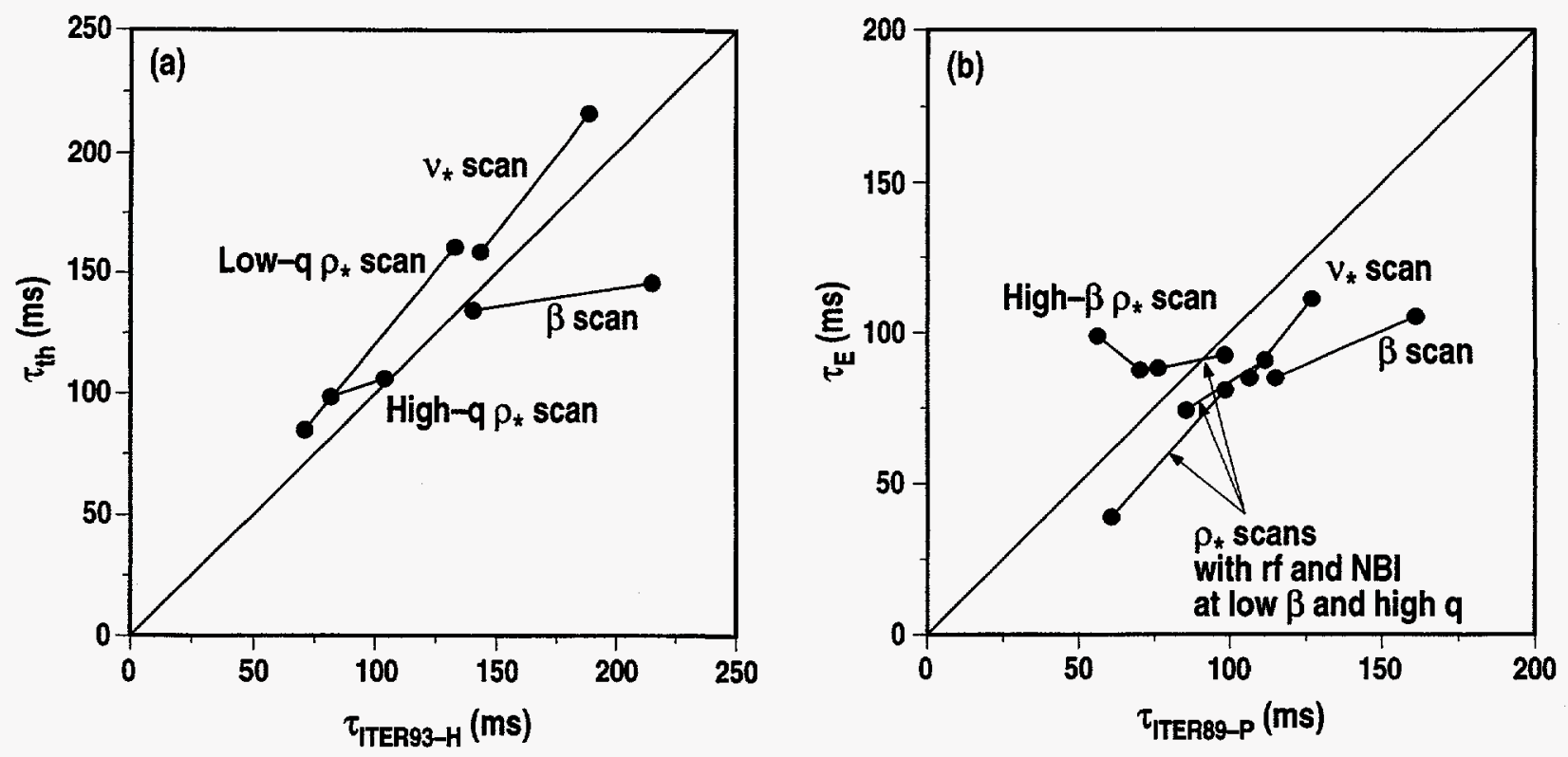

Fig. 4.2-1. Summary of measured global scalings. (a) H-mode dimensionless parameter scans; (b) L-mode dimensionless parameter scans.

\subsection{NEOCLASSICAL TEARING MODES}

The maximum beta which can be sustained for a long pulse in ITER-shaped plasmas in DIII-D with $\mathrm{q}_{95} \geq 3$, ELMs, and sawteeth is found to be limited by resistive tearing modes, particularly $\mathrm{m} / \mathrm{n}=3 / 2$ and $2 / 1$, where $\mathrm{m}$ and $\mathrm{n}$ are the poloidal and toroidal mode numbers, respectively. At low collisionality comparable to that which will occur in ITER, the beta limit is a factor of two below the usually expected $n=\infty$ ballooning and $n=1$ kink ideal limits. In FY96, the DIII-D database was filled in and expanded. Detailed analysis of the critical beta for $3 / 2$ and $2 / 1$ instabilities versus dimensionless local parameters $v_{*}$ for collisionality and $\rho_{*}$ for gyroradius are shown in Fig. 4.3-1. The onset for both modes scales as $v_{*}^{1 / 3}$ with the $\rho_{*}$ scaling uncertain between the 0 and $1 / 3$ power. The predictions for ITER are noted on the figure.

Two possible mechanisms have been evaluated as the cause of the onset of these instabilities. Resistive tearing modes that occur at rational surfaces $q=m / n$ cause reconnection into magnetic islands. The island onset and growth can be due to either free energy from an unstable current $J_{\phi}$ profile or to a helical bootstrap current which amplifies a seed island.

Resistive magnetohydrodynamic (MHD) analysis of $\mathrm{J}_{\phi}$ profile tearing stability is linearly computed from equilibrium fitting (EFIT) by analytical formulas and by the PEST-II and MARS codes. Resistive non-linear MHD analysis is computed on these equilibria with the PIES code. (The code work is part of an ongoing multi-institutional collaboration with the Princeton Plasma 
Physics Laboratory and the Ecole Polytechnique Federale de Lausanne in Switzerland.) Any changes to the current profile stability with beta and/or density may be due to current density profile modification by central beam-driven current and edge bootstrap and inductive currents. Sensitivity of both the linear and nonlinear code results to details of the current and q profiles makes comparison with experiment problematic.
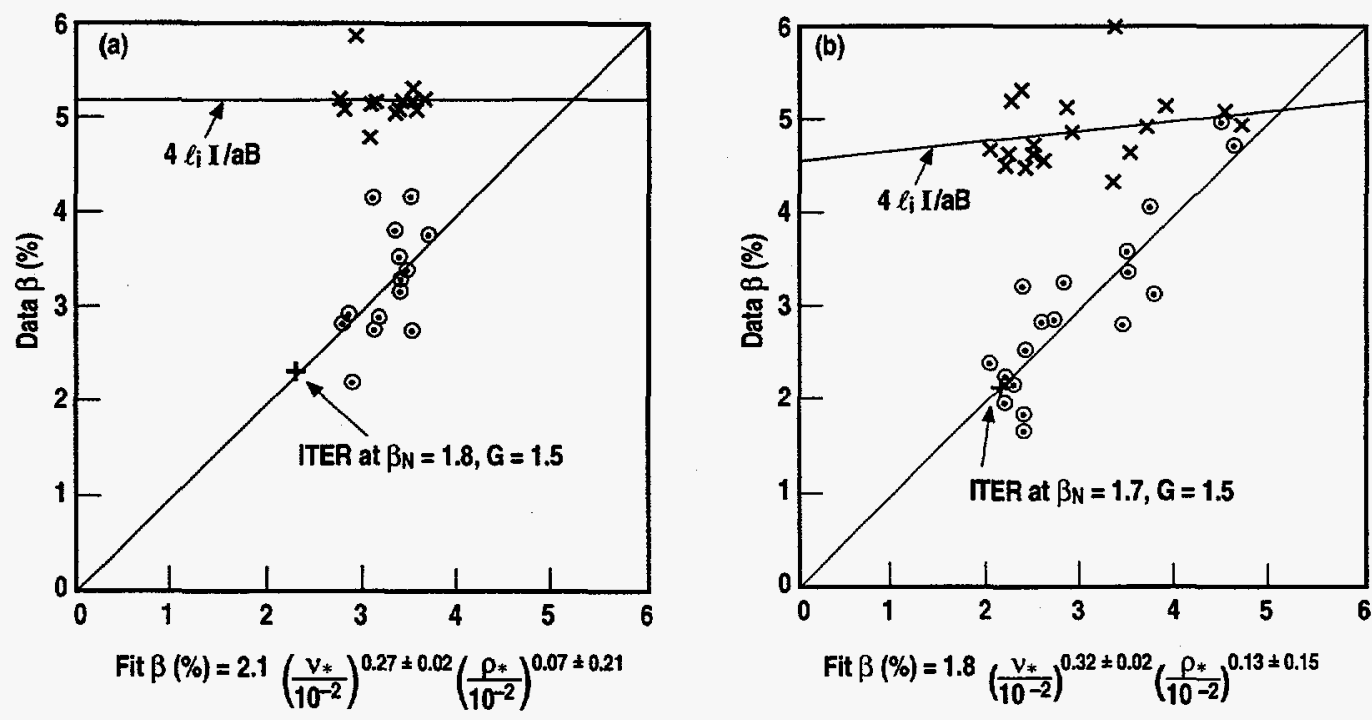

Fig. 4.3-1. (a) Onset of $3 / 2$ tearing (๑) in DIII-D fitted to local parameters. (b) Onset of $2 / 1$ tearing $(\odot)$ in DIII-D fitted to local parameters. Expected ITER beta limit is also shown (t) as well as expected ideal limit $(x)$.

An explanation of the experimental results can be made using the neoclassical bootstrap current destabilization of a seed island which is otherwise stable. This effect is increasingly more destabilizing with higher $\beta$. Other MHD events such as sawteeth or ELMs often trigger the onset of the resistive modes, supporting the idea that they are neoclassically destabilized by such a seed perturbation. The neoclassical destabilization of tearing modes requires the conditions to be right, i.e., high $\beta$ and low collisionality, and a seed island. Data was acquired in FY96 showing all the signatures of neoclassical destabilization. (An ongoing multi-institutional theoretical collaboration with the University of Wisconsin, the Institute for Fusion Studies at the University of Texas, and Culham Laboratory of England continued to be fruitful.)

\subsection{DISRUPTION STUDIES}

Considerable progress was made during FY96 in the disruption studies program. The major emphasis during the year was in the characterization and mitigation of the large poloidal halo 
currents and heat flux developed during disruptions. In both areas, progress was made both experimentally and in the detailed modeling of the processes.

One of the more significant disruption related problems for future machines is the large force due to poloidal halo currents that flow on the open field lines surrounding the plasma and return poloidally through the vessel. During FY96, a series of experiments were performed that carefully documented the amplitude and structure of these currents. During vertical displacement events (VDEs), poloidal halo currents up to $30 \%$ of the pre-disruption plasma current with a toroidal peaking factor of 2:1 were measured. However, significantly higher toroidal asymmetries occur at only slightly lower values of the halo current (Fig. 4.4-1). The toroidal structure of the mode was often observed to rotate between 200 to $400 \mathrm{~Hz}$, however, locking of the structure is sometimes observed. This slow rotation or locking implies that toroidally discrete vessel components must be designed for forces from the halo currents enhanced by the toroidal peaking factor.

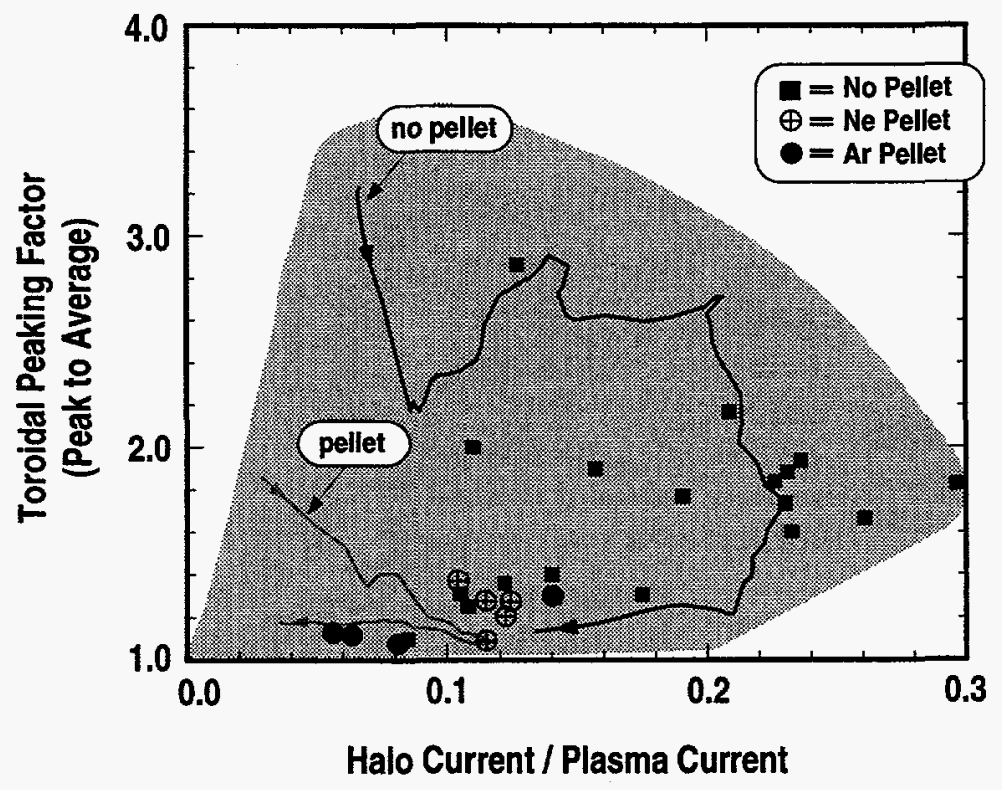

Fig. 4.4-1. Toroidal peaking factor and halo current normalized to the pre-disruption plasma current for VDEs. Points show the values at the time of peak halo current. The two solid lines show the trajectory of a pellet and non-pellet discharge with identical pre-disruption equilib ria. The shaded region is the boundary of the trajectories of all discharges.

An analytic model of the halo currents was developed in order to understand the large variations in the magnitude of the halo current at similar values of current and toroidal field. Results showed the current strongly depends on the current decay rate relative to the vertical instability growth rate. In particular, it was shown that if the current decay is slow relative to the 
growth of the vertical instability, large halo currents are developed. These results provided guidance in the development and successful understanding of experiments to reduce halo current during disruptions.

The technique that was pursued to reduce the large halo currents and the intense heat pulse during the disruption was the injection of impurity "killer" pellets. Injection of either neon or argon impurity pellets immediately following the onset of a VDE has successfully reduced the halo currents by $30 \%$ to $50 \%$, almost eliminated the toroidal asymmetry, and has simultaneously reduced the heat flux conducted to the divertor by at least $50 \%$ in reactor relevant discharges $(\mathrm{q}=$ $3.4, \beta_{n}=3.2$ ). Moreover, the reduction in both magnitude and toroidal peaking factor of the halo current are observed throughout the entire disruption, not just at the time of peak halo current (Fig. 4.4-1). The rapid and effective loss of thermal energy with pellet injection is confirmed by a 1-D model of the plasma including pellet ablation and impurity radiation. The model predicts that within $150 \mu \mathrm{s}$ after pellet ablation, both the electron and ion temperatures are at $10 \mathrm{eV}$ from initial values of $2 \mathrm{keV}$. These temperatures are confirmed using the multipulse Thomson scattering system. Despite the successful mitigation of halo current and heat flux, a critical problem created by the pellet injection is the formation of runaway electrons which are not typically observed on disruptions on DIII-D. Building on our understanding developed during FY96, the focus of the disruption program in FY97 will be the achievement of improved mitigation of both the halo currents and the heat flux to the divertor without the creation of runaway electrons.

\subsection{NONINDUCTIVE STARTUP EXPERIMENTS}

If a credible and robust technique can be developed to non-inductively ramp-up the plasma current for tokamaks, it will become possible to consider construction of a tokamak without an Ohmic heating solenoid. This would be particularly attractive for the spherical tokamak concept, where the solenoid is the main size constraint on aspect-ratio. Numerous techniques have been demonstrated or proposed to form tokamak discharges without use of a central solenoid; thus, breakdown and discharge formation appears to be a solved problem. The remaining challenge is to apply heating and current drive to this fledgling discharge and to increase the plasma current to a level at which the confinement and stability properties are typical of Ohmically driven plasmas. This demonstration will be major goal of the National Spherical Tokamak Experiment program at the Princeton Plasma Physics Laboratory and can be investigated by experiments on DIII-D.

This past year, a one day experiment was dedicated to the demonstration of ramp-up using neutral beam current drive and bootstrap currents. A number of new ideas and capabilities were 
employed to investigate the physics issues associated with noninductive startup. First, the diagnostic capabilities on DIII-D have now advanced to the stage where all of the important parameters governing resistivity and current drive can be measured. Motional Stark Effect (MSE), charge exchange recombination (CER) measurements of the carbon density profile for $Z_{\text {eff, and time }}$ resolved measurements of the electron temperature are all essential for understanding current drive. Second, analysis techniques have been established to determine the non-inductively driven current profile for comparison to theory. Finally, the plasma control system has advanced to the stage where the important parameters governing the driven current can be controlled in real-time; this list includes real-time control of the loop voltage waveform, rather than plasma current, and $\beta, \beta \mathrm{p}$ and $\beta_{\mathrm{N}}$ by neutral beam modulation.

The experiments, and subsequent analysis, showed that the ramp-up is inhibited by MHD instability at low plasma current. Measurements of the non-inductive current profiles and comparison with theory over a variety of conditions showed that tearing modes lead to anomalously low neutral beam driven currents, due to fast ion losses induced by magnetic islands. This experiment, like others being done (Section 4.3), showed that the steady-state beta limits imposed by neoclassical tearing modes are much lower than found transiently. For ramp-up, the tearing modes not only limit the power which can be injected, but also reduce the current drive efficiency.

Several methods will be tested in FY97 experiments to successfully achieve ramp-up. One is to increase the neutral beam driven current for a given beam power by increasing the ratio of electron temperature to plasma density. Another is to hold the plasma in L-mode so that more beam power can be injected for a given level of stored energy $(\beta)$. The greater beam power will also drive more current.

\subsection{PARTICLE TRANSPORT}

What determines the electron density profile in the tokamak is yet to be finally explained. To make progress in this area, the electron particle transport coefficient (diffusivity) is being measured under various conditions in DIII-D.

Electron particle transport coefficients for L-mode, ELM-free H-mode, and ELMing $\mathrm{H}$-mode have been measured using deuterium gas puffing into DII-D and modeling of the density rise after the $\mathrm{L}-\mathrm{H}$ transition. For L-mode and ELMing $\mathrm{H}$-mode plasmas, the diffusion coefficient (D) increases with $\rho$, the scaled minor radius coordinate rising at the edge to several times that of the center. The central values of $\mathrm{D}$ are about the same in both modes, but for L-mode the edge value is almost twice that for ELMing $\mathrm{H}$-mode. In ELM-free $\mathrm{H}$-mode, the central value of $\mathrm{D}$ is 
also about the same as in the other modes, but the edge value decreases to approximately one-fifth of the central value.

A physics based model which predicts the steady state density profile in L-mode tokamak plasmas has been developed and compared with measured profiles. Although some of the assumptions which go into the model are not yet proved, the agreement of the experimental data with the model is quite good.

In the area of helium transport in DIII-D, studies have shown the scaling of core helium diffusivity to be gyro-Bohm-like (i.e., core transport rate increases linearly with ion gyroradius) with all other non dimensional parameters held at their ITER value. This result combined with the fact that $\mathrm{D}_{\mathrm{He}} / \chi_{\text {eff }} \sim 1$ in these plasmas make it likely that $\mathrm{D}_{\mathrm{He}} / \chi_{\text {eff }} \sim 1$ in ITER, implying that helium exhaust will not be limited by core helium transport. 


\section{OPERATIONS}

\subsection{OVERVIEW}

During FY96, over sixteen weeks of operation was achieved while maintaining reasonable availability and completing all necessary maintenance and repairs. This was accomplished in spite of limited staff and resources. A short maintenance vent was completed in early February and at the end of the fiscal year, a major refurbishment was begun to install the upper radiative divertor, relocate and improve diagnostics, and complete badly needed calibration and maintenance tasks.

Major external systems refurbished include replacing the ancient outdated tokamak control computer including redeveloping most of the systems software, and beginning the work to replace the neutral beam (NB) control computer. The operation of the ICRF systems became routine in mid-year with $3 \mathrm{MW}$ or more power coupled to the plasma. The first $110 \mathrm{GHz}$ gyrotron at DIII-D, was put into operation with first coupling into tokamak plasmas at the end of the operating period.

Table 5-1 lists the basic DIII-D system specifications.

Table 5-1

DIII-D System Specifications

\begin{tabular}{l|l}
\hline & \multicolumn{1}{|c}{ Present } \\
\hline Vacuum vessel volume & $37 \mathrm{~m}^{3}$ \\
Major radius & $1.67 \mathrm{~m}$ \\
Minor radius & $0.67 \mathrm{~m}$ \\
Maximum toroidal field & $2.2 \mathrm{~T}$ \\
Vessel elongation ratio & 26 \\
Maximum plasma current & $3.0 \mathrm{MA}$ \\
NB power & $20 \mathrm{MW}$ \\
ECRF power & $1 \mathrm{MW}$, upgrade to \\
& $3 \mathrm{MW}$ at in progress \\
ICRF power & $(110 \mathrm{GHz})$ \\
Current flattop (divertor at 2 MA) & $6 \mathrm{MW}(30$ to $120 \mathrm{MHz})$ \\
\hline
\end{tabular}




\subsection{TOKAMAK OPERATIONS}

In FY96, DIII-D was operated for 106 days. This included 83 days for physics experiments and 23 days for system checkout, vessel conditioning with plasma, and diagnostic calibration. An additional 19 days were used for high temperature bakeout of the vessel. The machine availability was $77.3 \%$ during physics experiments and a total of 2435 discharges were fired. Despite the increasing number of diagnostics and operations systems on the vessel and the increasing age of the facility, this availability remained at the level of previous years (Fig. 5.2-1). The sources of downtime as a fraction of total scheduled time for experiments is shown in Fig. 5.2-2.

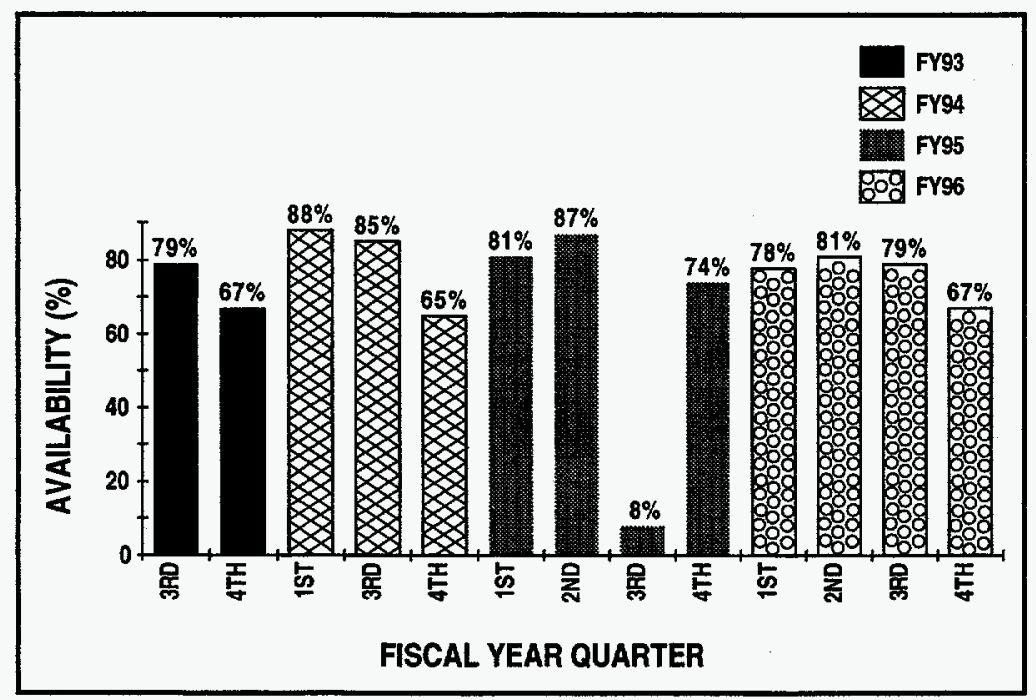

Fig. 5.2-1. Tokamak availability FY93 to present.

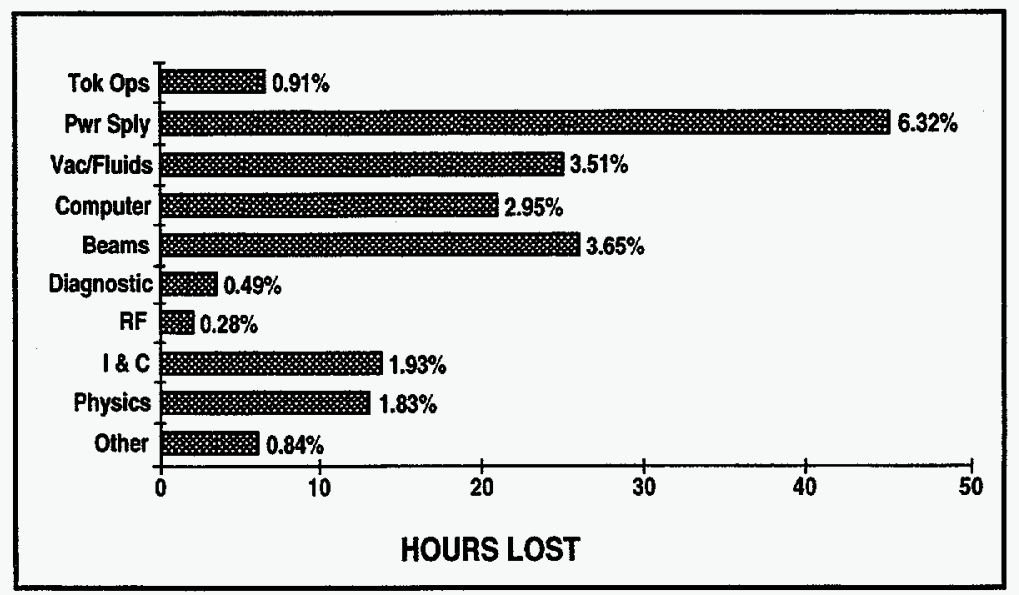

Fig. 5.2-2. Downtime for FY96. 
The chronology of events and highlights for FY96 is shown in Fig. 5.2-3. The major improvement in machine operations systems was the replacement of our outdated MODCOMP control computer with a modern UNIX-based computer and the addition of a user-friendly graphical interface. A significant advancement in plasma control was achieved through the implementation of real-time calculations of the magnetohydrodynamic equilibria on the plasma control system (PCS). This resulted in increased accuracy of our control parameters and ultimately will allow us to measure and control non-shape parameters such as the q-profile.

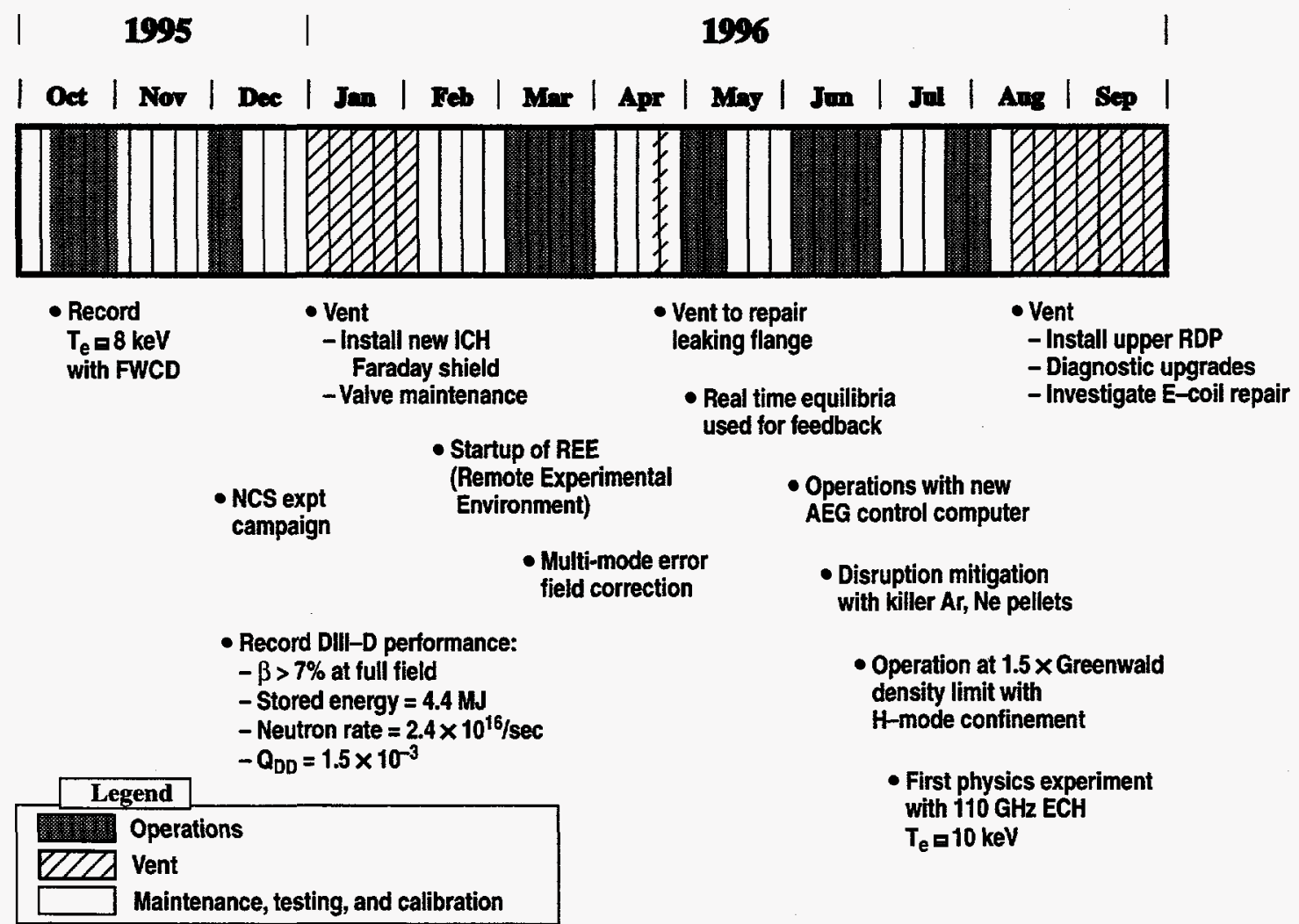

Fig. 5.2-3. DIII-D FY96 weekly operations schedule.

There were two major vents in FY96. The vessel was vented in January for the installation of a boron carbide-coated Faraday shield on the fast wave current drive antenna at $285 \mathrm{deg}$, refurbishment of the large torus isolation gate valves, and diagnostic upgrades. Following 12 weeks of operation, the vessel was vented in August and will remain open until March 1997 for the installation of the upper radiative divertor, diagnostic relocations to accommodate the new diagnostic upgrades and calibrations. 
Radiation levels at the site boundary were $9.3 \mathrm{mrem}$, well below the agreed site limits of $40 \mathrm{mrem} / \mathrm{yr}$. All operations were appropriately monitored for radiation levels and the program ALARA goals were net.

At the end of FY96, there were 1566 pieces of equipment being tracked by the Integrated Preventive Maintenance Program (IPMP). This is an increase of $6 \%$ over FY95. During FY96, 2475 tasks were completed in the IPMP.

Major support equipment was replaced. A large vapor degreaser for the vacuum cleaning area was ordered to replace the deteriorated, older unit. A new water chiller was installed to replace the freon cooling system for the six soft x-ray detectors installed on the tokamak. This eliminates the potential for freon leakage into the vessel. Two cooling water manifolds with 30 fully instrumented cooling circuits for calorimetry measurements were fabricated and installed in preparation for the $1 \mathrm{MW}$, Varian gyrotrons to be delivered in FY97.

Plans were developed for control and instrumentation upgrades for the cryogenic system to be implemented between FY97 and FY98. Various pieces of these upgrades are underway and procurement of the new programmable logic controller has begun.

Work has progressed throughout the year developing techniques for restoration of the full ohmic heating solenoid (E-coil) system. Half of the solenoid capability was disabled in FY95 due to water leakage from a cracked water cooled lead to the solenoid. The repair is underway to stop crack growth by clamping the lead to prevent additional flexing. The repair is occurring during the winter vent period exploiting limited access through a removed vertical port in the floor of the vessel. Access is limited and most work is being done using remote tools and borescope viewing. Successful completion should allow use of the full solenoid in late spring 1997.

The electrical systems performed reliably. The NB power systems were successfully operated at their full output voltage level in support of negative central shear experiments. A vertical plasma position control algorithm was implemented in the digital PCS. Problems with the emergency diesel generator and two $15 \mathrm{kV}$ vacuum circuit breakers were resolved without impacting the operations schedule.

Control boxes for five of field shaping coils (F-coils) were modified to accept an input which turns off the switched mode power supply, enabling a shape change from double null to single null during the same discharge. This greatly increases the flexibility of experimental operations on DIII-D. 
The digital PCS was upgraded to include six Supercards with five fast throughput daughter boards. There are now 208 analog input channels on the PCS, increasing the number of signals which can be used for real-time discharge control.

A Plasma Control Calibration Maintenance System database has been constructed for the purpose of facilitating access to calibration data and changes for the DIII-D electrical systems.

Software upgrades were implemented in the PCS. Analysis was completed to determine causes of observed differences in F-coil currents between fixed boundary equilibria in EFIT and free boundary fits of magnetics data for the same equilibria. An additional constraint equation was implemented allowing the two methods to apply the same constraints on the coil currents. Testing of PCS vertical position control software continued.

\subsection{NEUTRAL BEAM OPERATIONS}

Sixteen weeks and three days of plasma heating experiments were supported by NBs in FY96. Beam systems operated two additional weeks to condition ion sources, perform beam power calibrations, and support DIII-D vessel cleaning after a one-month vent in the second quarter. During the first quarter of FY96, six out of eight ion sources successfully operated at beam energy equal to or greater than the designed value of $80 \mathrm{keV}$, providing higher injected beam power to support an important physics experiment on beta limit and high performance extension of negative central shear plasmas.

Spatial mapping of the six plasma grid modules purchased from Burle Industries was completed. A technique for coordinate reference between the four modules of each grid and between the four grids of the ion source accelerator was developed. Two of the new plasma grid modules were installed on the spare accelerator and were aligned. This accelerator replaced one of the accelerators on the beamlines in January 1996 and was conditioned and is working very well.

Significant progress has been made in the upgrade of the NB computer systems. This includes completion of the control screens, beamline thermocouple display screens, demonstration of waveform plotting from within Accessware ${ }^{\mathrm{TM}}$, demonstration of loading and running the NB power supply timing sequence, functional testing of the ability to communicate the timing information between the new beam computer and the DIII-D physics timing interface program, acquisition of test signals applied to the NB digitizers, new scheme for eliminating the troublesome mode control panels, and a new layout for the NB control consoles. The NB system will start up with new computers and control consoles in March 1997. 
Availability of the NB system by month is shown in Fig. 5.3-1. The "Available" category is based on the beam system requirement requested by the physics experiments. The difference between the "Available" and "Injecting" categories represents beam systems which were available but were not used for injection during physics experiments. The various causes for downtime are shown in Fig. 5.3-2.

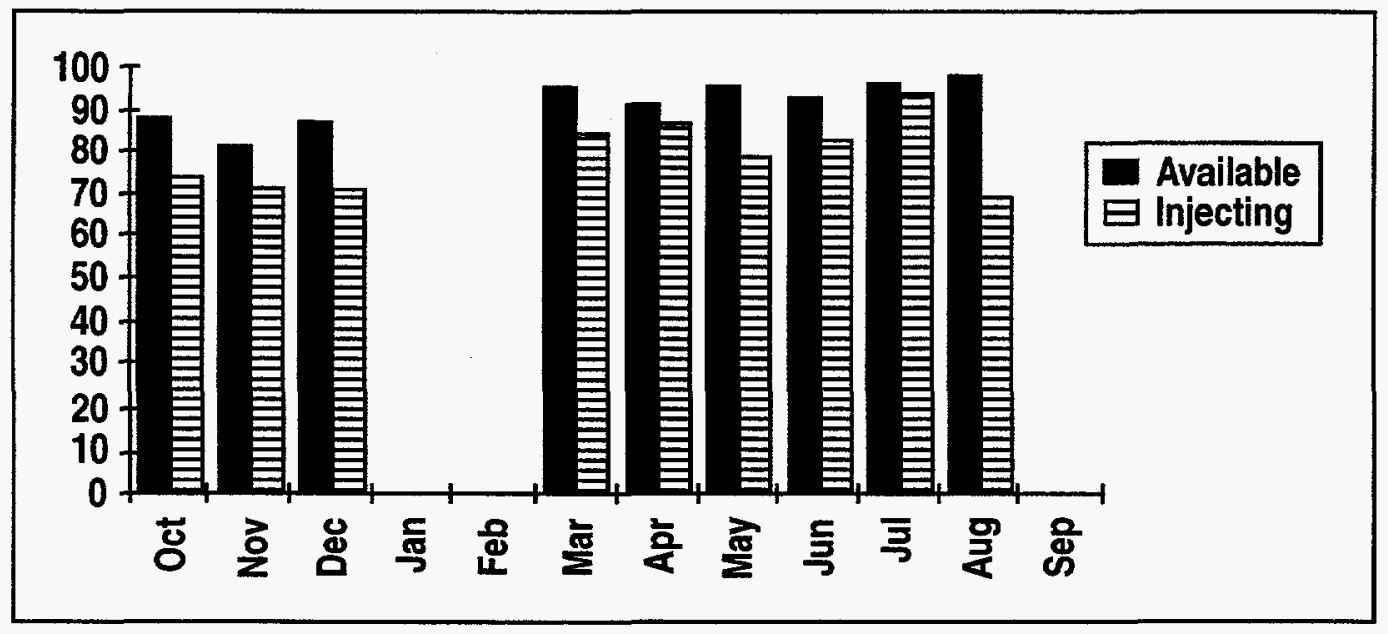

Fig. 5.3-1. NB availability by month.

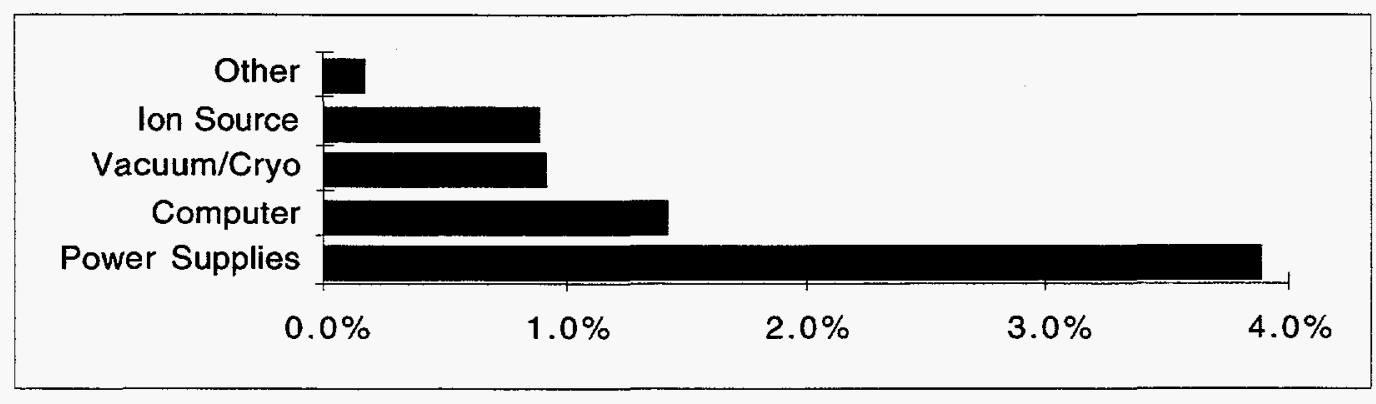

Fig.5.3-2. Causes of NB downtime by category (percent of total operating time).

\subsection{ICRF}

The DIII-D ICRF system consists of three four-strap antennas, fabricated by the Oak Ridge National Laboratory, and three high power transmitters. Each transmitter has a maximum output power of $2 \mathrm{MW}$. The tow newest transmitters were purchased from Asea Brown Boveri (ABB), and are referred to as $\mathrm{ABB}$ Nos. 1 and 2. This purchase, together with their two antennas, were an established Department of Energy ICRF Upgrade Project on DIII-D. 
The year started with the completion of the ICRF Upgrade Project by the installation of water cooled ferrites in the output cavities of the ABB transmitters. These water cooled ferrites were successful in suppressing the parasitic oscillations that were generated by the final power amplifier tetrode and allowed the system to operate at full $2 \mathrm{MW}$ level. By the middle of the year, all three ICRF systems were running well and over $3 \mathrm{MW}$ of radio frequency $(\mathrm{RF})$ power was delivered to the plasma. A total of 20 days of ion cyclotron resonance heating (ICRH) supported experiments were performed during the year.

As is common with new systems being brought on line, several areas requiring improvements or modifications were identified as the power levels were increased in the ICRF systems. The dc breaks in series with each antenna loop shorted at RF voltage levels below the nominal design values. Additionally, $\mathrm{RF}$ emitted radiation from the system was affecting the NB interlock system, which prevented the simultaneous operation of the NBs and the ICRF systems. Lastly, at peak power levels, the primary high voltage power supply feeding the final amplifier tetrode had too much reduction in voltage at turn on. All of these items were resolved during the year to reach the high power, reliable operation achieved during the final experimental operations period.

To solve the dc break arcing problem, all eight of the dc breaks were removed from the system and refitted with a modified center conductor and new insulation material. Following experimental operations in July, both ABB transmitters were run into vacuum (no plasma) in order to determine the ultimate RF voltage limits for each system. ABB No. 1 was run at power levels exceeding $40 \mathrm{kV}$ while $\mathrm{ABB}$ No. 2 had some pulses up to $36 \mathrm{kV}$. These levels are very satisfactory, exceeding the design level of $30 \mathrm{kV}$.

The NB interlock interference was found to be caused by an improper shielding termination technique used on the NB pyrometers. Retrofit kits were made up and installed, reducing the pickup to almost undetectable levels.

ABB No. 1 and No. 2 have been refitted with a new high-voltage regulator system. Initial results have confirmed that this design works well with only a $\pm 1 \mathrm{kV}$ swing between no load and full load.

\subsection{ECRF}

The commissioning of the gyrotron, purchased from the Russian company, GYCOM, started this year with the first operation being the testing of the gyrotron at $500 \mathrm{~kW}$ with $10 \mathrm{~ms}$ pulses 
into dummy load. Vacuum vessel "dummy" load conditioning of the gyrotron was then undertaken in preparation for plasma operations. The Russian gyrotron was successfully fired into several DIII-D plasmas on the last day of operations in 1996, nearly six months ahead of the schedule at that time.

During the initial testing of the gyrotron, a parasitic oscillation developed that caused the interlock system to shut down the tube prematurely. A Russian expert came to GA to assist in the elimination of the parasitic oscillation and help condition the gyrotron to long pulses. Reasonably reliable operation into a free-space, short pulse dummy load was achieved by installing both a capacitive filter and a magnetic shield near the cathode of the gyrotron. After incorporating better radio frequency interference (RFI) shielding methods on all interlock and RF detection signals, operation out to $200 \mathrm{~ms}$ (the temporary administrative limit imposed by the Russian experts until their return) was routinely obtained.

In May, the waveguide transmission run was reconfigured so that the gyrotron could be connected to the DIII-D machine or through the waveguide switch to the dummy load. Electron cyclotron heating power was injected into plasmas in June. Injected power was estimated to have been in excess of $500 \mathrm{~kW}$ and all pulses, including the first, were $200 \mathrm{~ms}$ in length and validated the use of the small diameter (1-1/4 in.) transmission line components. Central electron temperatures as high as $10 \mathrm{keV}$ were achieved for low density of $5 \times 10^{12} / \mathrm{cm}^{3}$. Both full power pulses and modulated pulses, with $50 \mathrm{~Hz}$ modulation frequency, were injected. The power deposition region was changed both by rotating the last mirror poloidally and by changing the magnetic field. The waveguide pressure remained low, showing that there are no regions where RF power is intercepting the transmission line components.

\subsection{COMPUTER SYSTEMS}

During FY96, there were 3821 tokamak shots. The largest shot contained $190.8 \mathrm{MB}$, and $412 \mathrm{~GB}$ of data were collected for the year (a $58 \%$ increase).

Increases in the shot size and in the number of users led to very heavy usage of the main analysis computers. System parameters often had to be modified to handle these increases. Two 9 GB drives were installed for utilization by computer users, and various file systems were expanded in size. The shot storage space was doubled from 20 to $40 \mathrm{~GB}$ to help accommodate the ever increasing shot size and an increase in the amount of data to remain in the active area. 
A major upgrade took place for the tokamak control computer system from the old 16-bit minicomputer to a modern UNIX computer system. This upgrade involved considerable work to develop user friendly graphical interfaces and to develop procedures written in the $C$ language for the sequencing and control of the tokamak shot. The new system was commissioned in June and ran very well for the four weeks of operations that month.

Near the end of the fiscal year, work began in earnest to upgrade the NB computer systems to the newer UNIX platform. Software to be used is a combination of the control software from the operations computer and the data acquisition software from the data acquisition computer. Completion is expected by spring of 1997.

Significant software work went to converting a number of user codes from the older VAX computer to the newer UNIX systems. These codes included a large variety developed over many years on DIII-D. Codes used in tokamak operations, diagnostic acquisition and display, and equilibrium and transport analyses. A project was begun to transfer data from thousands of shots that were on thousands of bpi reel tapes, to $8 \mathrm{~mm}$ tapes which are the current medium used for archiving.

\subsection{DIAGNOSTICS}

The DIII-D diagnostic effort in FY96 focused on preparing for the considerable changes required and desirable additions to the diagnostic set with the installation of the upper hightriangularity advanced tokamak divertor. The engineering design and preparation for these changes was largely completed in FY96 with fabrication and installation commencing in late FY96. A list of the diagnostic systems affected by the divertor installation and the additions or improvements to diagnostics that were started in FY96 is presented in Table 5.7-1. An important addition to the DIII-D diagnostic set that will be completed in early 1997 is the new radial Motional Stark Effect (MSE) diagnostic (see also Section 8.2). This additional view (the existing MSE diagnostic has two ports with views optimized for the edge and the core) will allow a measurement of both the current profile and the radial electric field.

While our main thrust in diagnostics in FY96 has been related to the upper divertor modifications, several other improvements to diagnostic systems have been made. A single frequency (tunable 85 to $95 \mathrm{GHz}$ ) reflectometer was installed to give some measurement of the central density during negative central shear discharges. The reflectometer measures the electron density at the center, $n_{e}(0)$, at a single time during the density ramp as $n_{e}(0)$ passes through the cutoff density of the reflectometer. 
The installation of the vertical array of charge exchange particle analyzers was completed and the system commissioned. This diagnostic will provide information on the profile of fast ion distributions. Parts of the edge charge exchange recombination system upgrade were made operational and collected high quality tokamak data. Two spectrometers (eight spatial channels) were used with the newly developed charge coupled device cameras. The electronics and shielding of these systems will continue to be optimized in FY97.

Table 5.7-1

Diagnostic Changes During the 1996-1997 Winter Vent

\begin{tabular}{|c|c|}
\hline Divertor Modifications & New Capability \\
\hline Bolometer & Core Profile \\
\hline Soft $x$-ray imaging arrays & Radial MSE \\
\hline RF probes & Central CER channel \\
\hline Neutron detectors & Disruption Studies \\
\hline Electron cyclotron emission (ECE) & Expanded tile current arrays \\
\hline \multirow[t]{14}{*}{ Ion cyclotron heating $(\mathrm{ICH})$ antenna camera } & Runaway electron infrared (IR) camera \\
\hline & Divertor \\
\hline & Magnetic probes \\
\hline & Upper div. fast pressure gauges \\
\hline & $\begin{array}{l}\text { Tang. div. visible survey instrument, poor } \\
\text { resolution, extended domain (SPRED) }\end{array}$ \\
\hline & Upper div. throat L.P. \\
\hline & Lower div. throat L.P. \\
\hline & ITER Demonstration \\
\hline & Tangential interferometer \\
\hline & Fast wave reflectometer \\
\hline & Miscellaneous \\
\hline & Midplane fast pressure gauge \\
\hline & Viewing \\
\hline & Radial particle analyzer \\
\hline
\end{tabular}

Table 5.7-2 lists the major diagnostic systems operating on DIII-D at the end of FY96 along with the quantities measured. In addition to the development of new diagnostic systems, we continue to refurbish diagnostics that are known to be operating with critical components near or beyond their life expectancies. 
Table 5.7-2

Plasma Diagnostics at the End of FY96

\begin{tabular}{|c|c|c|}
\hline Diagnostic & Quantity Measured & Comments \\
\hline \multicolumn{3}{|c|}{ Operations-Related Diagnostics } \\
\hline Magnetics & $\dot{B}$, Magnetic flux $I_{p}$ & $\begin{array}{l}2 \text { poloidal ( } 58), 1 \text { toroidal (8) mag. Probe } \\
\text { arrays with } 50 \mathrm{kHz} \text { response; } 41 \text { flux loops, } \\
9 \text { diamagnetic loops, } 30 \text { saddle loops, } 3 \mathrm{I}_{\mathrm{p}} \\
\text { Rogowskis }\end{array}$ \\
\hline Hard X-rays & & $\begin{array}{l}2 \text { toroidal locations, } 4 \text { detectors, } 1 \mathrm{kHz} \\
\text { response }\end{array}$ \\
\hline Plasma TV & Visible light & Radial view, divertor, $\mathrm{RF}$ antennas \\
\hline IR cameras & Heat load to armored surfaces & $\begin{array}{l}\text { Upper divertor; inside wall, } 2 \text { toroidal } \\
\text { locations, lower divertor }\end{array}$ \\
\hline $\mathrm{H}_{\alpha}$ monitors & $\mathrm{H}_{\alpha}$ radiation, recycling & $\mathrm{H}_{\alpha}$ filtered, 16 locations, $10 \mathrm{kHz}$ response \\
\hline Neutron detectors & Fusion and photo-neutrons & 3 toroidal locations, $200 \mathrm{~Hz}$ response \\
\hline Soft $X$-ray arrays & Internal fluctuations & 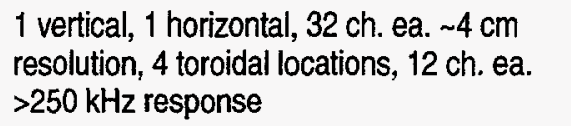 \\
\hline
\end{tabular}

\section{Electron Profiles}

Multipulse Thomson profile

$T_{e}(r, t), n_{e}(r, t)$

$\mathrm{CO}_{2}$ interferometers

ECE Michelson

Microwave radiometer

Fixed frequency reflectometer $\bar{n}_{e}(t)$

$T_{e}(r, t)$

$T e(r, t)$

$n_{e}(0)$
7 lasers, $6 \mathrm{~ms}$, vertical profile, $40 \mathrm{pts} .,<1 \mathrm{~cm}$ in edge resolution, $10 \mathrm{eV}>\mathrm{T}_{e}>20 \mathrm{keV}$

Vertical: 3 chords; radial: 1 chord

Radial profile, each $25 \mathrm{~ms}$

$32 \mathrm{ch}$ (ORNL), $0.2 \mathrm{~ms}, 2 \mathrm{~cm}$ resolution

Single pt. single time central density (UCLA)

\section{Fuctuations}

Microwave reflectometer

$$
n_{e}(r, t), \tilde{n}_{e}(r, t)
$$

UCLA collaboration - broadband; system gives profile in $5 \mathrm{~ms}$, narrowband system, $400 \mathrm{kHz}$ bandwidth

Correlation reflectometer

Radial correlation lengths in $\tilde{n}$

UCLA

FIR scattering

$$
\tilde{n}_{e}(r, t)
$$

UCLA collaboration $-4 \mathrm{MHz}$ bandwidth

$\mathrm{CO}_{2}$ scattering

$\tilde{n}_{e}(r, t)$

Fast magnetic probes

$\tilde{B}_{\theta}$

MIT collaboration - phase contrast $1 \mathrm{kHz}$, $100 \mathrm{MHz}$ bandwidth

$1 \mathrm{MHz}$ resolution, 2 arrays totaling 7 probes

Beam emission spectroscopy

U. Wisconsin collaboration, 16 spatial channels 
Table 5.7-2

Plasma Diagnostics at the End of FY96 (Continued)

\begin{tabular}{|c|c|c|}
\hline Diagnostic & Quantity Measured & Comments \\
\hline \multicolumn{3}{|l|}{ lon Temperature and Rotation } \\
\hline $\begin{array}{l}\text { Charge exchange } \\
\text { recombination }\end{array}$ & $\begin{array}{l}T_{i}(r, t), v_{\phi}(r, t) \\
v_{\theta}(r, t)\end{array}$ & $\begin{array}{l}16 \text { channels bulk plasma; } 16 \text { channels edge } \\
\text { plasma; } 3 \mathrm{~mm} \text { Ti edge resolution; } 6 \mathrm{~mm} v_{\phi} \\
v_{\theta} \text {, edge resolution }\end{array}$ \\
\hline \multicolumn{3}{|l|}{ Impurities and Boundary Parameters } \\
\hline Visible bremsstrahlung & $Z_{\text {eff }}(r, t)$ & Radial profile, 16 chords, $1 \mathrm{kHz}$ \\
\hline Bolometer arrays & Radiated power & $\begin{array}{l}\text { Tomographic reconstructions, } 2 \text { arrays, } \\
60 \mathrm{ch} ., 1 \mathrm{kHz}\end{array}$ \\
\hline Graphite foil bolometers & Heat load to the wall & $\begin{array}{l}7 \text { on the outer wall, } 2 \text { inside wall, } 2 \text { lower } \\
\text { divertor, } 1 \text { upper divertor }\end{array}$ \\
\hline SPRED (dual range) & Impurity concentrations & $\begin{array}{l}280-1200 \AA ; 100-290 \AA \text {, spectrum every } \\
1 \mathrm{~ms} \text {; edge profiles with scanning capability }\end{array}$ \\
\hline$H_{\alpha} T V$ & Divertor $H_{\alpha}$ & LLNL \\
\hline Divertor IR Cameras & Heat load to divertors & $\begin{array}{l}\text { LLNL, GA, } 125 \text { ms for a profile, upper, } \\
2 \text { lower, ADP throat }\end{array}$ \\
\hline Langmuir probes & Edge $T_{e}(t), n_{e}(t)$ & On divertor tiles \\
\hline Fast stroke Langmuir probe & Edge $T_{e}(t), n_{e}(t)$ & SNL, UCLA \\
\hline DiMES calorimetry & Surface erosion & Divertor Material Exposure System \\
\hline Penning gauges & He pressure & $\begin{array}{l}\text { ORNL - He pressure under the divertor } \\
\text { baffle }\end{array}$ \\
\hline Divertor reflectometer & Peak $n_{e}$ in divertor & $2 \mathrm{ch}$. in lower divertor (LLNL) \\
\hline Divertor Thomson scattering & $n_{e}(r), T_{e}(r)$ in divertor & $\begin{array}{l}1 \text { laser, } 50 \mathrm{~ms}, 8 \text { pts., }<1 \text { eV temperature } \\
\text { sensitivity (LLNL, GA) }\end{array}$ \\
\hline Divertor SPRED & Impurity concentrations in divertor & LLNL \\
\hline Visible SPRED & Impurity concentrations in divertor & Many chords \\
\hline$X$-point fast stroke probe & $T_{e}(r), n_{e}(r)$ in divertor & UCSD, SNL \\
\hline Fast pressure gauges & Neutral pressure & $\begin{array}{l}\text { Near divertor region (ASDEX, LLNL), } \\
\text { toroidal array, } 2-3 \text { ms response }\end{array}$ \\
\hline Tile current monitors & Poloidal tile currents & $\begin{array}{l}\text { Includes tile currents in the ADP, toroidal } \\
\text { array }\end{array}$ \\
\hline $\begin{array}{l}\text { Multichannel divertor visible } \\
\text { spectrometer (MDS) }\end{array}$ & Divertor impurity concentrations & ORNL -7 channels \\
\hline Divertor Baratron gauge & Neutral pressure & $\begin{array}{l}\text { ORNL - verified ASDEX gauge } \\
\text { measurements }\end{array}$ \\
\hline
\end{tabular}




\section{Table 5.7-2}

\section{Plasma Diagnostics at the End of FY96 (Continued)}

\begin{tabular}{|c|c|c|}
\hline Diagnostic & Quantity Measured & Comments \\
\hline \multicolumn{3}{|l|}{ Fast Ion Diagnostics } \\
\hline Scintillator & Neutron fluctuations & $50 \mathrm{kHz}$ response time \\
\hline Neutral particle analyzers & Charge exchange particles & $\begin{array}{l}4 \text { electrostatic analyzers, } 1 \text { horizontal, } 3 \\
\text { vertical (UCI, PPPL) }\end{array}$ \\
\hline Fusion products & $\begin{array}{l}\text { Fast neutrons, tritons } \mathrm{He}^{3} \text {, other } \\
\text { fast jons }\end{array}$ & UCl collaboration; movable probe assembly \\
\hline \multicolumn{3}{|l|}{ Current Drive Diagnostics } \\
\hline Motional stark effect (MSE) & $B_{p}(r)$ & $\begin{array}{l}16 \text { radial channels; } 2 \mathrm{~cm} \text { resolution, } 5 \mathrm{~ms} \\
\text { response }\end{array}$ \\
\hline ECEMichelson & Microwave emission & $\begin{array}{l}\text { Detects tail population in the electron } \\
\text { distribution function, profile in } 25 \mathrm{~ms} \\
\text { (Maryland) }\end{array}$ \\
\hline SXR pulse height & High energy $X$-ray spectrum & $\begin{array}{l}\text { Russian collaboration; detects tail } \\
\text { population in electron distribution; } \\
\text { spectrum every } 100 \mathrm{~ms}\end{array}$ \\
\hline RF probes & RF radiation & $\begin{array}{l}\text { Phase and amplitude of ICRH RF on inside } \\
\text { wall }\end{array}$ \\
\hline
\end{tabular}




\section{PROGRAM DEVELOPMENT}

\subsection{ECH UPGRADE PROJECT}

The Electron Cyclotron Heating (ECH) Upgrade project consists of two $1 \mathrm{MW} \mathrm{ECH}$ systems, each consisting of a $110 \mathrm{GHz}$ gyrotron connected to a quasi-optical launcher via an evacuated low loss corrugated transmission line. Two different gyrotron vendors are used. The first gyrotron was purchased from GYCOM, a Russian company and is shown in Fig. 6.1-1. The second gyrotron will not be delivered until early 1997 and is coming from CPI (formerly known as

Varian). Commissioning of the GYCOM system was substantially completed in 1996, culminating in the successful demonstration of electron heating to $10 \mathrm{keV}$.

A fast responding dummy load was developed to tune-up the GYCOM gyrotron at DIII-D. This could be used for both power measurements and wave beam alignment. The alignment issue arose from the measurements in Russia that identified that the radio frequency (RF) beam was not centered on the exit window and deviated from the normal direction by about $1 \mathrm{deg}$. Therefore, before the mirror interface tank containing the two focusing/correcting mirrors could be mounted to the gyrotron, the RF beam path had to be determined accurately. A short pulse air load was designed and installed in an anachoic chamber. This load has a Teflon front surface plate that is faceted with square pyramids. The back plate is made of polymethyl-pentene [Tokamak Physics Experiment (TPX)]. Between the two plates is octanol, a liquid which has an attenuation of $25 \mathrm{~dB}$ through the approximately $1 \mathrm{~cm}$ gap. The temperature difference between the octanol inlet and outlet is used to measure the total RF power absorbed. Heat sensitive paper was mounted to this load to measure the beam intensity pattern. Figure 6.1-2 shows the measured pattern at $1724 \mathrm{~mm}$ from the window produced by two 2 ms long pulses.

The evacuated ECH waveguide used on DIII-D imposes constraints on the location and adjustments of the interfacing mirrors. The interfacing mirrors are housed in an Mirror Optical Unit (MOU) that must maintain a vacuum of $10^{-4}$ Torr or better, provide for remote adjustment and alignment of the mirrors, remove the energy absorbed by the mirrors, and adsorb all stray RF power not coupled to the exit waveguide. To perform the gyrotron-to-waveguide interface, the two interface mirrors were first located in the MOU on a plane common to the gyrotron window 


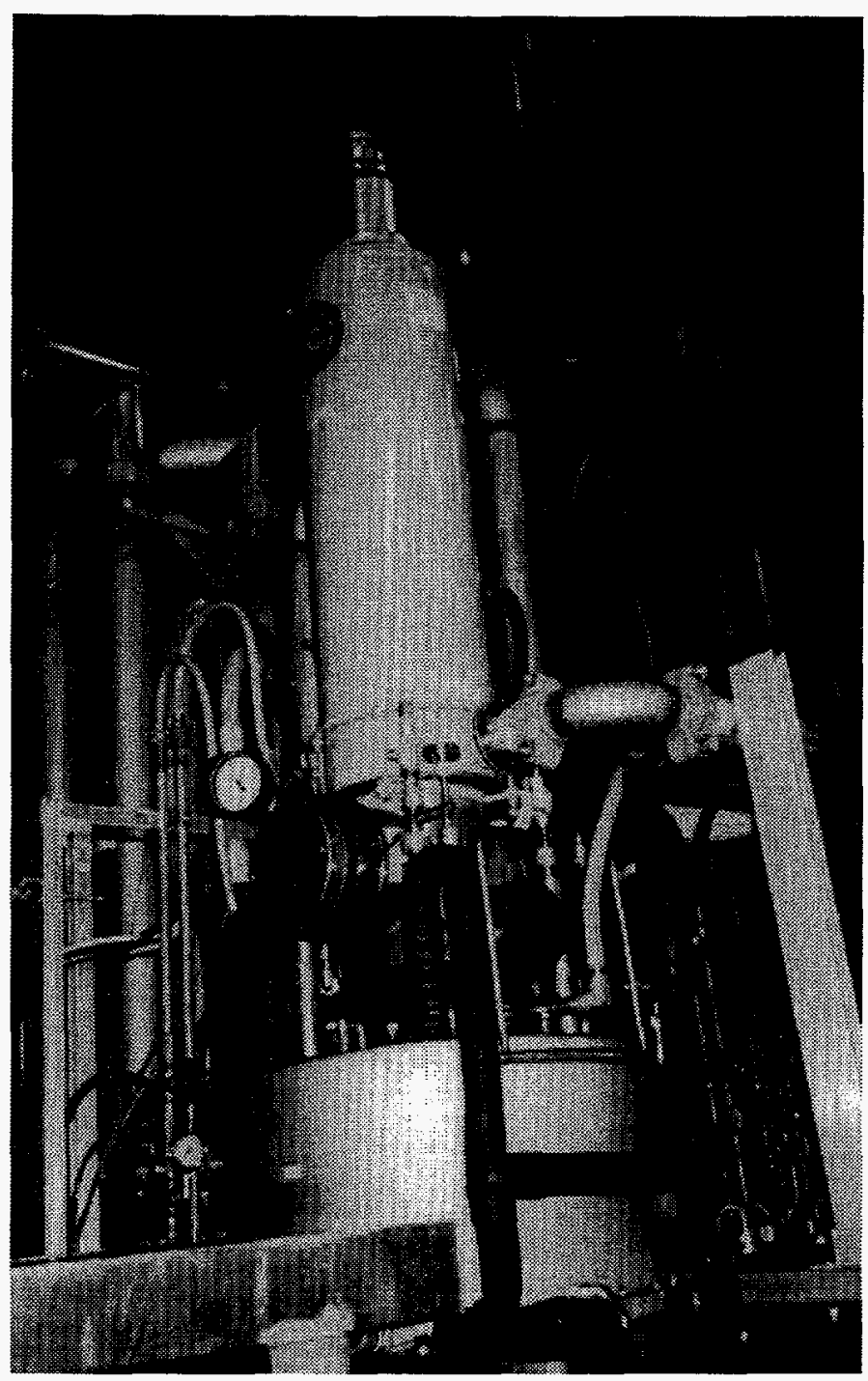

Fig. 6.1-1. $110 \mathrm{GHz}$ GYCOM gyrotron installed in DII-D ECH vault.

center line and the exit waveguide centerline (Fig. 6.1-3). The MOU was aligned to match the RF beam path. As determined by patterns on burn paper placed at the two ends of the waveguide (Fig. 6.1-3), the position of the exit waveguide (angular and offset) was adjusted to place the center of the RF at the center of the entrance and exit of the waveguide. Repetitive pulses into the short pulse load demonstrated that over $750 \mathrm{~kW}$ was coupled through the waveguide.

Pulses up to $200 \mathrm{~ms}$ have been successfully achieved with power measured into the dummy load of $586 \mathrm{~kW}$ with the gyrotron operating at $70 \mathrm{kV}$ and $30 \mathrm{~A}$. This corresponds to an efficiency of $29 \%$, which is close to the $31 \%$ measured in Moscow. Little effort has yet been taken to optimize the gyrotron magnetic field settings, so this agreement is good. 


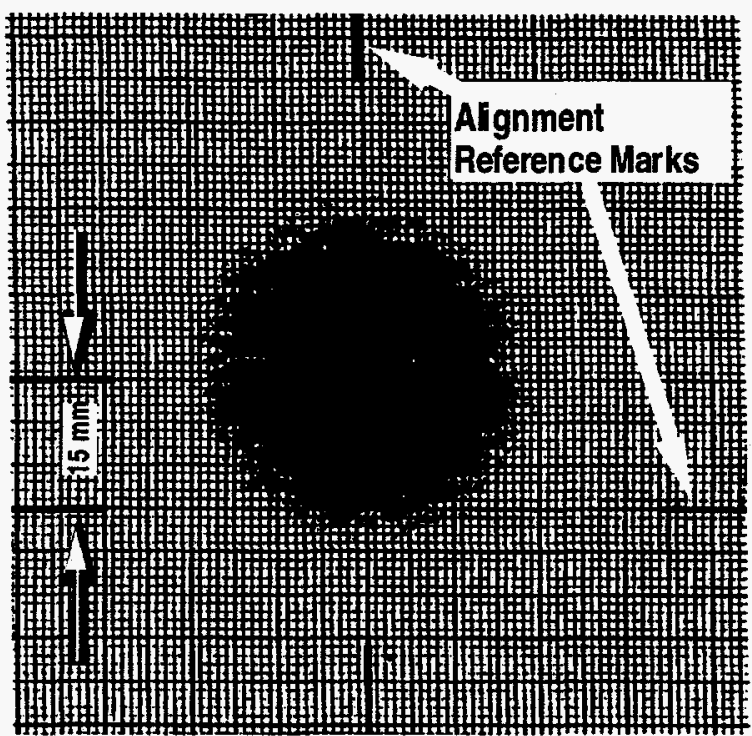

Fig. 6.1-2 RF burn pattern $1724 \mathrm{~mm}$ from gyrotron window.

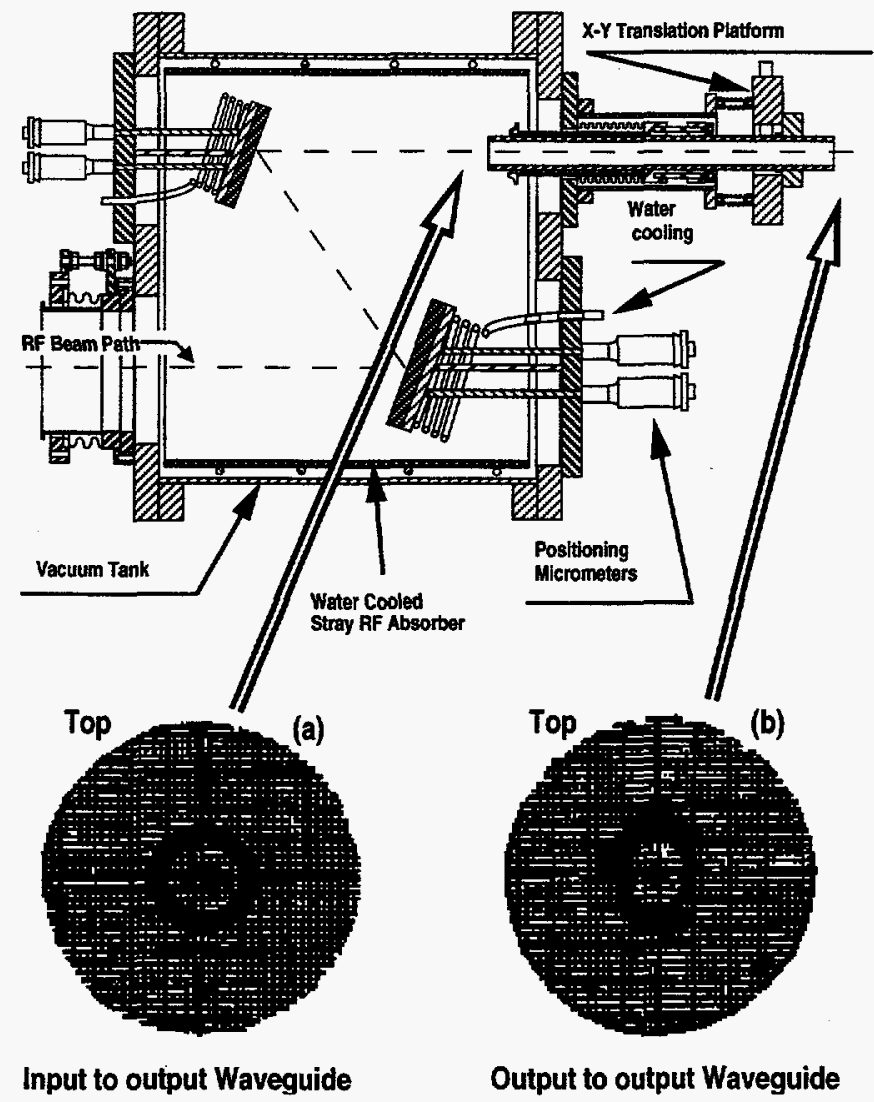

Fig. 6.1-3. MOU which consists of: the interface mirrors, mirror positioning equipment, vacuum tank, stray RF absorber, and the output waveguide assembly. Also shown are the RF burn patterns at (a) the input, and (b) the output of the MOU exit waveguide. 
ECH power was injected into plasmas for the first time in June. Injected power was estimated to have been in excess of $500 \mathrm{~kW}$ and all pulses, including the first, were $200 \mathrm{~ms}$ in length. Central electron temperatures of $10 \mathrm{keV}$ were achieved for low density of $5 \times 10^{12} / \mathrm{cm}^{3}$. Both full power pulses and modulated pulses, with $50 \mathrm{~Hz}$ modulation frequency, were injected. The power deposition region was changed by rotating the last mirror poloidally and by changing the magnetic field.

Everything for the second ECH system has been fabricated and installed. The RF transmission system for the CPI gyrotron in the DIII-D machine pit and the run into the ECH gyrotron vault has been completed. All miter bend mirrors have been installed and the complete transmission line to the DIII-D machine has been leak checked. All heater blankets and thermocouples have been installed and water cooling to the miter bends is being installed. The gyrotron superconducting magnet purchased from Oxford Instruments arrived and has been successfully tested. The gyrotron high voltage tank and controls have been installed and the water cooling manifold and instrumentation system has been fabricated.

The remaining tasks to be performed to complete the project are: (1) receipt of the CPI gyrotron with installation and mating to the transmission line, and (2) fabrication of the mirrors for the MOU. CPI will perform this last task since it requires detailed knowledge of the wave pattern exiting the gyrotron. The present schedule has the gyrotron being delivered in early 1997 with checkout of the gyrotron to start in March 1997.

\subsection{RADIATIVE DIVERTOR UPGRADE PROJECT}

The goal of the Radiative Divertor Project (RDP) is to install a new divertor in DIII-D. The main new features of the RDP are that it can pump high-triangularity plasma shapes and it has a more closed (baffled) divertor. Previous data has indicated that this better isolation between the core and divertor plasmas can improve plasma performance.

Due to funding limitations, a phased implementation plan has been developed for the divertor installation (Fig. 6.2-1). The final installation (right-hand side of Fig. 6.2-1) will have baffles and pumping at the four strikepoints of a double-null, high triangularity plasma. Either single- or double-null configurations can be run. The first phase (Phase 1A, left hand side of Fig. 6.2-1) involves the installation of the upper pump and the upper outer baffle. During FY96, we finalized the design, built and tested a prototype of the water-cooled baffle plates, and began installation of Phase 1A of the RDP. The width of the outer baffle was set at the value determined by the codes, 
although it can be varied in the current Phase 1A from this optimal condition by moving the outer strike point (but is more restricted in the final, more baffled case).

The upper cryopump, which is similar to the existing lower cryopump, has been designed and constructed. The external cryosystem that supplies the liquid helium to the pump has been fully designed and the construction began at the end of FY96.

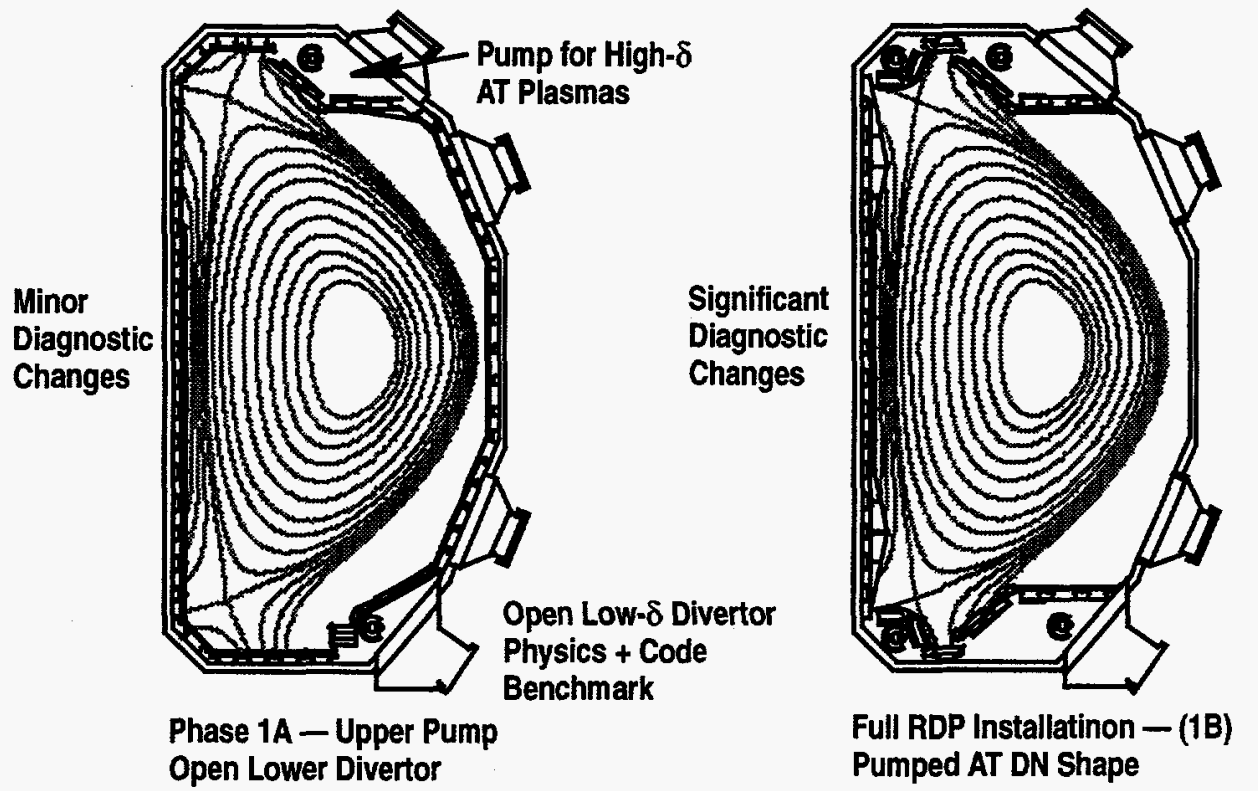

Fig.62-1. We will employ a phased approach in implementing the RDP.

In Phase 1A, we will retain the existing (open) lower divertor and all of its diagnostics, particularly the divertor Thomson Scattering. This will allow us to continue detailed physics measurements in lower single-null, low triangularity plasma shapes. We will have limited diagnostics in the upper divertor: an ASDEX gauge from the Oak Ridge National Laboratory (ORNL) to measure the baffle pressure and thereby the plasma exhaust, several Langmuir probes to measure the ion flux near the pump baffle, $\mathrm{H}_{\alpha}$ photon emission measurements (ORNL), some bolometer measurements of radiated power with the existing bolometer array, and three gas injectors. We will be able to make several core measurements to assess the effects in the core of gas injection and neutral atom shielding in the edge. These Langmuir probes were developed at the national laboratories at Livermore and Sandia.

At the end of FY96, nearly all of the engineering issues (e.g., halo current loads, disruption loads, interfaces with diagnostics) were resolved. We have paid particular attention to the vacuum 
isolation between the pump chamber and the plasma. Gas seals are located at all the joints of the panels and chimneys have been installed for vertically-viewing diagnostics.

We expect that the RDP installation will be complete in March of 1997 and that experiments focused on using this new configuration will start in the Spring of 1997.

\subsection{VANADIUM DIVERTOR STRUCTURE}

General Atomics is implementing a plan for the utilization of a low activation vanadium alloy in the DIII-D tokamak. The goal of this plan is to demonstrate the design, fabrication, and operation of a water-cooled vanadium alloy structure in the DIII-D radiative divertor upgrade.

A V-4Cr-4Ti alloy, identified in the U.S. as a candidate alloy for future use in fusion applications, was melted and processed into sheet and rod product forms for use in fabricating a component of the DIII-D divertor structure. Analysis of V-alloy specimens exposed to DIII-D vacuum and cooling water side environments to verify the compatibility of the alloy under DIII-D operating conditions indicated no degradation of material properties. Research and development of welding methods applicable to the fabrication of the V-alloy structure were continued, and successes were achieved in producing ductile, high-strength, vacuum leak-tight joints by all of the methods under investigation including resistance seam bonding, friction welding, and explosive bonding. 


\section{SUPPORT SERVICES}

\subsection{QUALITY ASSURANCE}

Fusion Quality Assurance (QA) engineers, inspectors, and support personnel maintained a high level of activity during 1996. Significant projects supported were preparations for the E-Coil repair, the GYCOM (Russian) gyrotron installation, and the Radiative Divertor Project.

The QA group performed the following specific jobs:

- Design support for ECRF, ICRF, diagnostics and in determining the extent of manufacturing variations in the major and minor radii and angle of water cooled panels.

- Performed receiving, source inspections, and measurements of purchased and fabricated material, parts, subassemblies and assemblies. Inspection activity was particularly heavy for the Radiative Divertor Project.

- Completed routine quarterly column and footing subsidence tasks and the semi-annual pit wall crack inspections.

- Developed and implemented the Fusion Group training program. A total of 163 Fusion Group employees and collaborators received the training.

- Implemented the Continuous Improvement Committee established by the Senior Vice President. Continuous Improvement is a means by which every member of an organization continually examines all of the processes, procedures, methods and activities by which work is accomplished, and implements or suggests constructive changes. The Committee is made up of nine members representing the different organizations that make up the Fusion Group. The Committee is chartered to receive, analyze, and make recommendations on all Continuous Improvement Opportunities (CIOs) which cannot be immediately resolved by line management. The Committee met three times during this reporting period to define the Continuous Improvement process, discuss how to get Fusion Group personnel to participate in the program and to address the CIO forms 
received. The Committee has received $17 \mathrm{CIO}$ forms. Four of the forms have been closed out. Each of the remaining forms has been assigned and is being addressed.

\subsection{PLANNING AND CONTROL}

The Planning and Control group supported operation and maintenance of the DIII-D facility. Planning and Control provided long-term program planning, as well as day-to-day scheduling (cost control, preparation of Field Work Proposals, and Cost and Fee Proposals), processing of purchase requests, expediting and reporting of status. These support activities are essential to constraining the program within prescribed budgets and schedules. Our planning activities (budget, schedule, resource) enabled us to maximize the utilization of available resources for accomplishment of program goals and were important in planning and replanning of scope, budget, and schedule with fluctuating funding levels.

Major planning activities during FY96 included work on the 1 MW Russian ECH system, new AEG computer system, Radiative Divertor Project (RDP) diagnostic relocation, E-coil lead repair and numerous collaborator diagnostic modifications and upgrades and vent that started in August of 1996 and centered around the installation of the radiative divertor.

\subsection{ENVIRONMENT SAFETY AND HEALTH}

The fusion safety program provides for the safe operation of the DIII-D facility and for a safe working environment for employees and visitors. Special programs address high voltage and high current, high vacuum systems, ionizing radiation, microwave radiation, cryogenics and the use of power equipment and machine tools. DIII-D is provided support by GA's Licensing, Safety and Nuclear Compliance organization and GA's Safety organization in areas such as health physics, industrial hygiene, environmental permitting, hazard communication, hazardous waste, and industrial safety.

The Fusion Safety Committee focuses on addressing both and longer range safety needs and goals. The Safety Committee meets twice a month and solicits specialized help from any of the five fusion safety subcommittees during reviews of lasers, electrical systems, vacuum systems, the use of cryogens or chemicals. In addition, two individuals are dedicated full-time to on-site "preventive" safety involvement. Their activities include writing and reviewing procedures, developing and conducting special training classes, conducting inspections and follow-up, and providing continuous oversight to assure compliance with established safety policies, procedures, and regulations. 
The DIII-D Emergency Response Team consists of individuals involved directly with maintenance and operation of the DIII-D equipment. They are trained in cardiopulmonary resuscitation (CPR), first aid, use of self-contained breathing apparatus (SCBA) and the use of fire extinguishers, evacuation and crowd control, and facility familiarization. The team can respond within. seconds to provide immediate assistance until outside emergency assistance arrive.

All new employees and collaborators must go through a thorough and comprehensive safety indoctrination by the Senior Fusion Safety Officer and the Pit Coordinator. They are informed of the specific potential hazards that are present daily at DII-D and the special safety precautions and rules that apply, with specific emphasis on the areas where they will be working. Subcontractors also receive a similar indoctrination.

Training is all-important to the safety of both personnel and equipment. Due to the complexity of the DIII-D site and its potential hazards, numerous safety training classes are conducted. Subjects of the classes included: confined space entry, back injury prevention, radiological safety, laser safety, electrical and high voltage safety, hazard communication and hazardous waste disposal, cryogenic safety, crane and forklift operation, lockout/tagout, machine shop tool usage and basic industrial safety requirements.

Safety inspections are conducted throughout the year to promote an active hazard prevention program. The inspections are conducted by a combination of Fusion, GA Licensing Safety and Nuclear Compliance personnel and outside consultants.

Five internal safety inspections of the DIII-D site were conducted by representatives from GA Safety, Fusion Safety, Fusion Management, and Fusion Facilities Engineering with a total of 105 discrepancies noted. At the close of FY96, there were zero items remaining on the discrepancy list.

The San Diego Fire Department's Combustible, Explosive and Dangerous Materials (CEDMAT) team inspected the DIII-D facility.

DOE-OAK conducted a safety review of the DIII-D site in April and mentioned 19 items that needed attention or correction. At close of FY96, there is still active progress on four remaining items. DOE inspectors gave DII-D an overall excellent rating.

The Fusion Safety Committee met 24 times this fiscal year to discuss various safety issues. The committee reviewed and approved 12 Hazardous Work Authorizations (HWA) after appropriate recommendations and changes by the Safety Committee and select safety subcommittees, and 
reviewed three incidents that involved no injury and three accidents that required minor off-site medical treatment. There were no lost time accidents. The committee also reviewed two DIII-D safety procedures.

\subsection{VISITOR AND PUBLIC INFORMATION PROGRAM}

Tours of the DIII-D facilities are open to organizations and institutions interested in fusion development (colleges, schools, government agencies, manufacturers, and miscellaneous organizations). These tours are conducted on a noninterference basis and are arranged through the DIII-D tour coordinator whose responsibilities include security, arranging tour guides, and scheduling tours. During 1996, 1,853 people toured DIII-D for a total of 16,474 during the last ten years.

DIII-D personnel have also taken an active role in supporting science education within the community. The partnership between the fusion group and Kearny High School included student and teacher internships, donation of surplus equipment, school visits and lectures by staff members and participation by students and teachers in GA-sponsored projects. These activities, primarily undertaken by volunteers, have enriched the educational program at Kearny and in the community at large and resulted in the naming of the GA-Kearny High School partnership as the 1996 outstanding partnership in San Diego. 


\section{COLLABORATIVE EFFORTS}

\subsection{DIII-D COLLABORATION PROGRAM OVERVIEW}

The General Atomics (GA) DIII-D tokamak and its fusion research program has become a true national users facility. The total number of national and international collaborators on DIII-D in FY96 was over 200 including both full and part time collaborators. Through our national and international collaboration programs, DIII-D is playing a major role in the worldwide advancement of fusion research towards the goal of fusion science and energy. Our collaboration with universities and Department of Energy (DOE) national laboratories has brought new recognition to our program and broadened its scope. DIII-D international collaborations have helped the U.S. to maintain its international role as a strong contributor to International Thermonuclear Experimental Reactor (ITER).

In particular, collaborations with Japan focussed on tokamak optimization issues associated with steady state operation. Collaborations with Joint European Torus (JET) were productive in leading to a better understanding of the "Negative Shear" high confinement mode. Our collaboration with Russian fusion research institutes and scientists has helped maintain an active Russian fusion program as well as providing GA and the U.S. fusion program with additional DIII-D relevant research. Our program of long term exchanges with China has proved beneficial to both parties.

The DIII-D collaborative programs have contributed to the results presented in the previous sections of this report and are discussed in more detail in the following.

\subsection{LAWRENCE LIVERMORE NATIONAL LABORATORY (LLNL)}

The LLNL Collaboration contributed to both the Divertor and Advanced Tokamak Programs on DIII-D during FY96. Significant progress was made both in physics analysis and in diagnostic design and implementation.

The divertor diagnostics, notably the Divertor Thomson Scattering (LLNL), the bolometer (GA/LLNL), the divertor SPRED (LLNL), the infrared television (IRTV) system (LLNL), and the 
Tangential TV system (LLNL) were used to develop a new picture of the edge plasma which was presented as an invited talk at the November American Physical Society meeting (Fig. 8.2-1). The measured divertor temperature is low $(\sim 2 \mathrm{eV})$, recombination may be important near the divertor plate, and conduction cannot explain the heat flow to the divertor plate. We also used the divertor survey instrument, poor resolution, extended domain (SPRED) to determine that most of the radiation is due to carbon even when other impurities have been injected. The UEDGE and DEGAS codes were used extensively to model all of these data. The UEDGE solutions indicate that chemical sputtering may be the most important production mechanism (as opposed to physical sputtering). A complete impurity model was added to UEDGE for these runs.
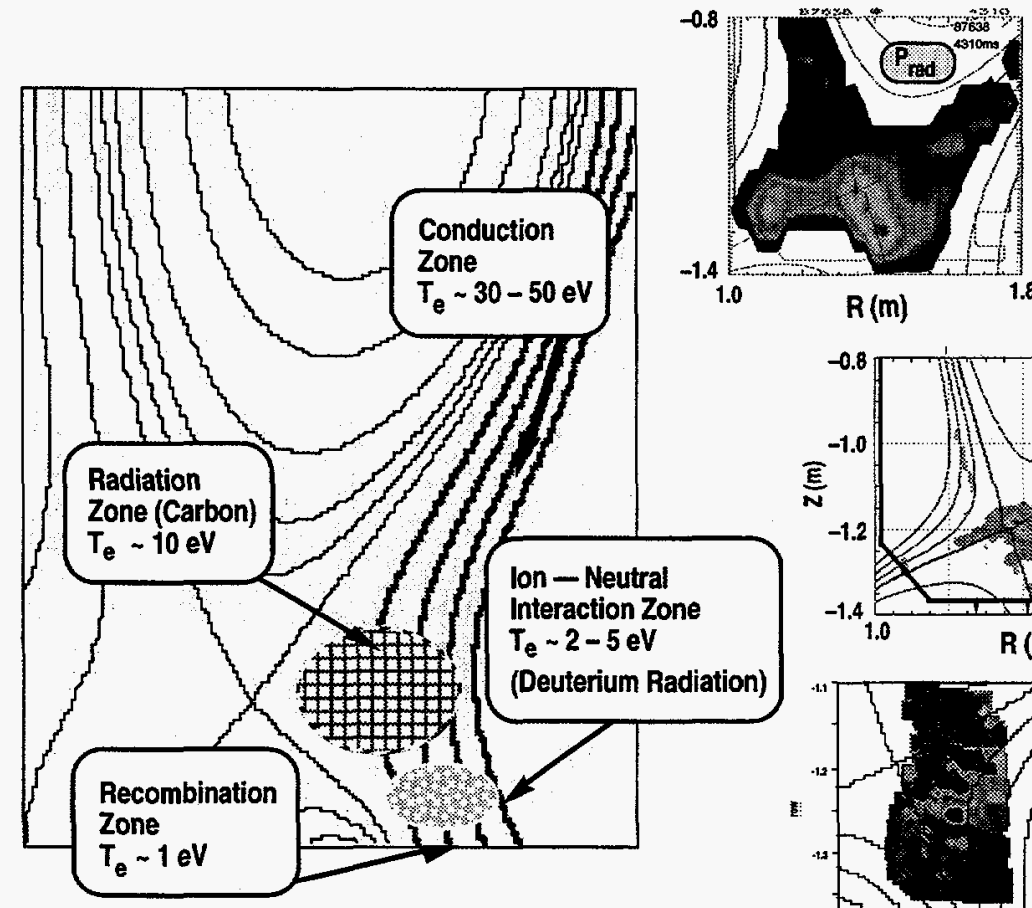

2-D Bolometer Inversion of Radiated Power

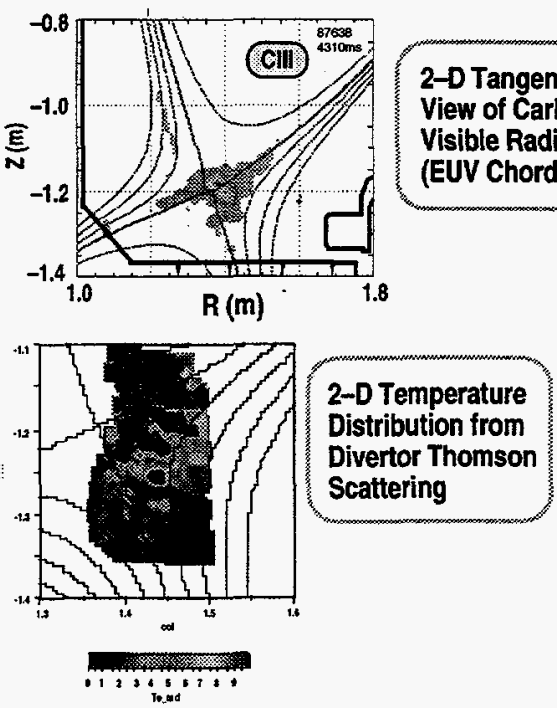

2-D Tangential

View of Carbon

Vible Radiation

UV Chord Vlew)

Fig. 8.2-1. We are developing a physics model of the detached divertor plasma with new 2-D diagnostic data.

In the Advanced Tokamak area, our focus is on measurement and control of the current profile and measurements of transport. The multi-channel Motional Stark Effect (MSE) diagnostic operated routinely and was used with equilibrium fitting (EFIT) to measure the $J(r)$ profile. It was determined that the electric field in the plasma was an important "correction" to this data under some circumstances. For this reason, in FY96 we designed and are constructing an upgraded MSE system (Fig. 8.2-2) that can measure both the J(r) and E(r) profiles simultaneously. These 
data will be compared with the plasma rotation data obtained from the charge exchange recombination (CER) diagnostic.

Data analysis and Remote Experimental Site operation have also been an important part of the LLNL Collaboration. LLNL has helped to develop several tools that are currently being used for improved communications both within the DIII-D site and externally.

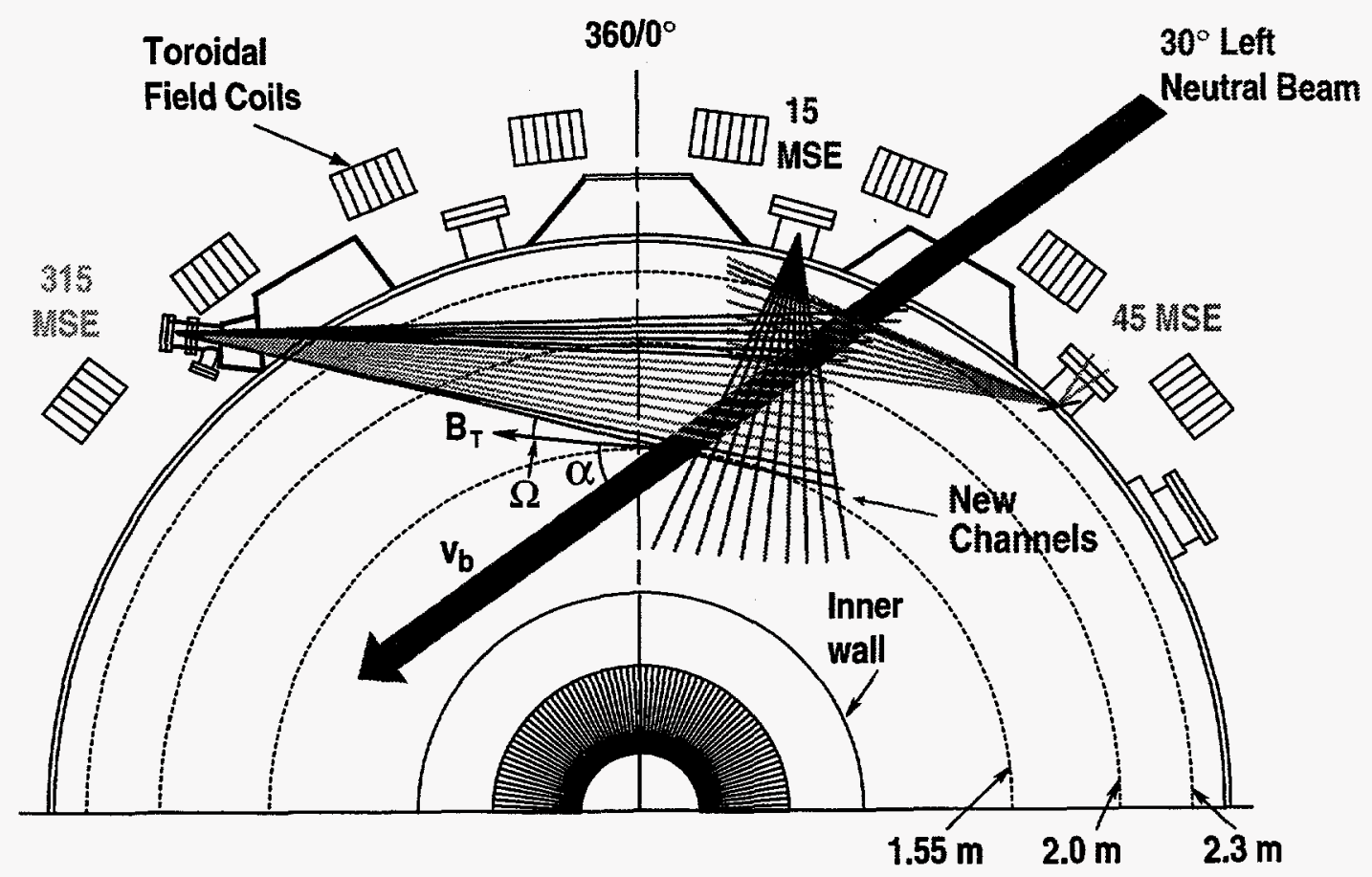

Fig. 8.2-2 MSE $E_{r}$ upgrade viewing geometry.

\subsection{OAK RIDGE NATIONAL LABORATORY (ORNL)}

ORNL scientists are continuously on site at DIII-D and the engineering teams, and other scientists support DII-D activities from Oak Ridge. The main areas of collaboration for ORNL on DIII-D in 1996 were particle transport and control and ion cyclotron range of frequencies (ICRF) physics and technology.

The ORNL main ion particle control program was focused in several areas: (1) density limits, (2) wall inventory multi-machine comparisons, (3) particle balance in negative central magnetic shear (NCS) plasmas, and (4) edge plasma/neutrals modeling. ORNL scientists provided an important leadership role in the highly successful density limits campaign which both isolated important density limit processes and demonstrated the existence of an operational window 
with good $\mathrm{H}$-mode confinement and density 1.5 times the Greenwald density "limit." In addition, a new topic of study was started. The impact of neutrals on $\mathrm{H}$-mode transport barrier formation ties together ORNL's expertise on transport barrier formation and edge plasma modeling. The initial results from this effort are: (1) the charge exchange damping of neutrals on the plasma main ion rotation is largest near the $\mathrm{X}$-point where the core fueling and neutral density in the main plasma are the highest, and (2) the presence of a localized damping term in the X-point area can dominate magnetic pumping effects locally and cause strong changes in the calculated poloidal flow patterns. Both of these effects can have a large impact on the $\mathrm{L}-\mathrm{H}$ transition power threshold, among other things.

In the area of helium transport, studies have shown the scaling of core helium diffusivity to be gyro-Bohm-like (i.e., core transport rate increases linearly with ion gyroradius) with all other nondimensional parameters held at their ITER value. This result combined with the fact that $\mathrm{D}_{\mathrm{He}} / \chi_{\text {eff }} \sim 1$ in these plasmas make it likely that $\mathrm{D}_{\mathrm{He}} / \chi_{\text {eff }} \sim 1$ in ITER, implying that helium exhaust will not be limited by core helium transport. In the divertor area, analysis of "puff and pump" results show that induced scrapeoff layer (SOL) flow is effective in increasing the divertor enrichment of impurities with the largest increase (approximately a factor of 3) observed for argon. Also, analysis of edge localized mode (ELM)-free discharges (ELM-free $\mathrm{H}$-mode, VH-mode, and NCS H-modes) show unabated buildup of carbon during the ELM-free phase, with the electron source rate in these plasmas primarily coming from this carbon influx.

During FY96, the ORNL pellet injector began routine operation with more than 3000 pellets injected into DIII-D discharges. The primary physics areas explored were density limit exploration and disruption mitigation studies. The $2.7 \mathrm{~mm}$ pellet repetition rate was doubled from 5 to $10 \mathrm{~Hz}$ giving a throughput of about 90 torr-liters/s. With this improvement, a plasma density of 1.5 times the Greenwald density limit was achieved for $600 \mathrm{~ms}$. During this period, the energy confinement time was $>75 \%$ of the ITER-93H value. For the disruption mitigation studies, neon and argon pellets were produced. Both pellet varieties reduced "halo" currents and vacuum vessel forces by $50 \%$. In both cases, the plasma thermal energy (as measured by the soft $\mathrm{x}$-ray arrays) was dissipated on the time scale of the pellet ablation time $(0.5 \mathrm{~ms})$.

In the ICRF area, a replacement Faraday shield was built and installed for the 285 to $300 \mathrm{deg}$ fast wave antenna. The new shield features a single tier of horizontal rods with a plasma vapor deposited boron carbide coating. Inspection of the shield during the fall vent showed no apparent degradation of the coating. The reliability of the 0 and $180 \mathrm{deg}$ antennas was improved by the replacement of the center conductors of all eight dc breaks. $3 \mathrm{MW}$ of radio frequency (RF) power was coupled to ELMing $\mathrm{H}$-mode plasma in one experiment. The 0 and $180 \mathrm{deg}$ antennas have 
now been conditioned in vacuum to voltages exceeding the design criterion of $30 \mathrm{kV}$. Prototype fast arc/ELM discriminator hardware was installed on the 285/300 deg antenna. The discriminator is based on detection of the phase changes between forward and reflected power signals measured at different locations in the RF transmission line.

Fast wave current drive was modeled with the PICES full-wave ICRF modeling code based on measured plasma profiles and TRANSP-calculated beam ion slowing down spectra. General agreement between the modeling and the experiment was seen in fast wave driven current density in profile shape and amplitude (Section 3.3), scaling of current drive efficiency $\left[\mathrm{T}_{e}(0) / \overline{\mathrm{n}}_{\mathrm{e}}\right]$, slightly higher current drive efficiency for counter phasing than for co, and peaking of the fast wave power deposition profile as the toroidal field was decreased (with RF modulation measurements). Results of the toroidal field scan were analyzed to identify competing energy loss channels through resonant ion absorption by deuterium beams ions at high cyclotron harmonics and/or residual hydrogen ions at the second harmonic. Current drive efficiency predicted by modeling at lower toroidal field was somewhat higher than in the experiment and may be indicative of the presence of RF-driven tails.

\subsection{UNIVERSITY OF CALIFORNIA, LOS ANGELES (UCLA)}

UCLA participation in the DIII-D program expanded during FY96 and now includes activities in the areas of $\mathrm{H}$-mode physics, core turbulence and transport, $\mathrm{RF}$ heating and current drive, and boundary and divertor studies.

Some of the significant results obtained by the UCLA program on DIII-D during the year are the following. First, core plasma turbulence is strongly suppressed in high performance NCS plasmas, correlating with an improvement in transport and confinement. Obtaining the highest performance NCS plasmas, where ion transport is reduced to neoclassical levels across the entire plasma volume, is correlated with turbulence suppression across the entire plasma radius. Next, a major advance in basic plasma science understanding has been achieved by measurement of the electric field induced in the plasma by the fast wave ICRF heating and current drive system. Comparison of the measured RF induced electric field profiles with those predicted by RF modeling codes at ORNL show general agreement, though some discrepancies suggest that additional wave damping mechanisms may be active (i.e., mechanisms not included in the codes). Finally, another major advance has also been obtained in the development of reflectometer systems for density profile measurement; a new solid-state fast sweep reflectometer system implemented on DIII-D has demonstrated unprecedented levels of combined spatial and temporal resolution. The 
new reflectometer system provides accurate and reliable edge density profiles with $100 \mu$ s time resolution and sub-cm spatial resolution. As shown in Fig. 8.4-1, the system resolution is now sufficient to track edge density profile changes through giant ELMs for the first time. This improved system performance was obtained via a better basic understanding of how to optimize reflectometer performance based on radar system design principles.

\subsection{UNIVERSITY OF CALIFORNIA, SAN DIEGO (UCSD)}

Scientists from the Fusion Energy Research Program (FERP) at UCSD participate in the DIII-D program in three general areas: $\mathrm{H}$-mode physics, divertor physics, and disruption database and experiments. FERP staff have lead responsibility for the compilation and maintenance of a disruption database as a U.S. ITER R\&D task, in collaboration with: GA, Massachusetts Institute of Technology (MIT), Princeton Plasma Physics Laboratory (PPPL), and Los Alamos National Laboratory (LANL). In addition, FERP staff collaborate with Sandia National Laboratory (SNL) in providing two reciprocating Langmuir probe arrays, one on the outboard midplane and one in the lower divertor, for the DIII-D program. Data from these probes are used in a wide range of experiments carried out on the DIII-D tokamak. UCSD FERP staff are responsible for design, maintenance, operation, and upgrade of all non-mechanical components for these two probe drives, including power systems and supplies, interlock and control systems, signal conditioning and digitizing electronics, and data storage, archiving, and analysis hardware and software.
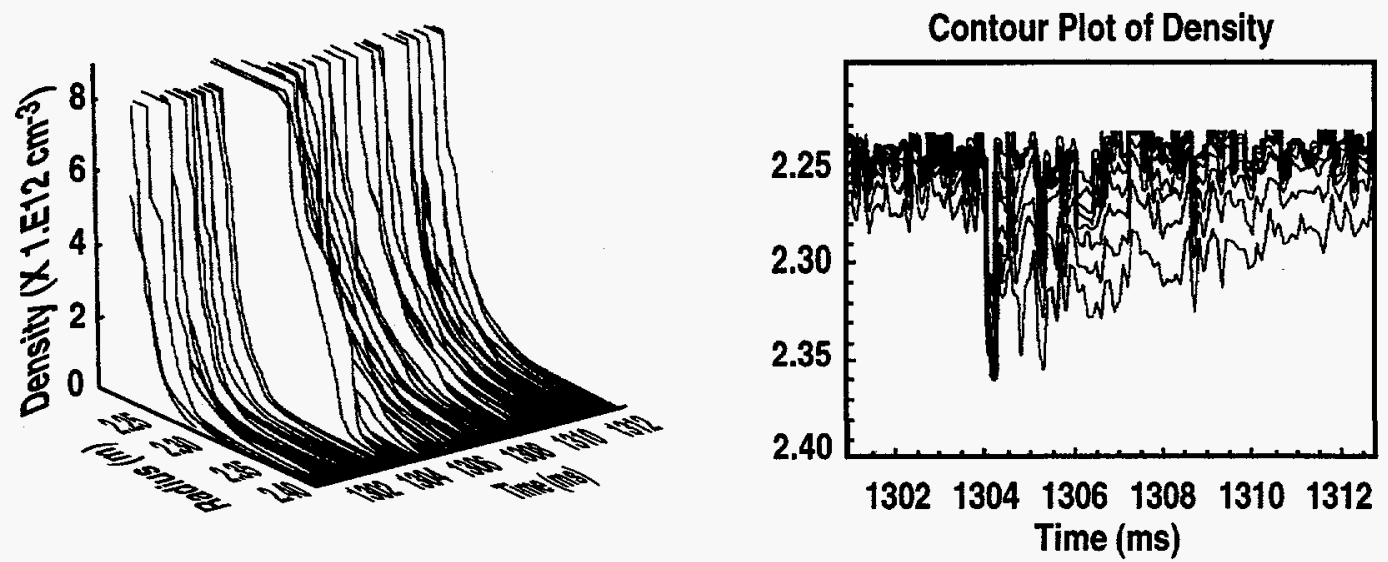

Fig. 8.4-1. Example of edge density profile evolution through a giant ELM as measured by the new fast sweep profile reflectometer system. 
In FY96, FERP staff participating in the DIII-D program focused on: (1) study of very slow L-to-H transitions, (2) study of the role of turbulence and turbulent transport in the SOL and divertor plasma, (3) compilation and maintenance of an ITER disruption database, (4) study of runaway electron production in density limit disruptions and "killer pellet" disruption mitigation experiments, (5) development of a fast magnetic flux surface reconstruction code TRITON for following the evolution of the magnetic configuration throughout a vertical displacement event or disruption, (6) study of plasma edge conditions and their effect on operation at and above the Greenwald density limit, and (7) support of efforts to characterize the divertor plasma conditions in attached and partially detached plasmas. Results of this work were presented at several meetings throughout the year, including: 9th Transport Task Force meeting (March 1996), the 12th International Meeting on Plasma-Surface Interactions (May 1996), the ITER Disruption Experts Group Meeting (April 1996), E.U.-U.S. Joint Workshop on Transport in Fusion Plasmas (September 1996) and the annual meeting of the Division of Plasma Physics of the American Physical Society (November 1996).

The goals of the FERP program at DIII-D in FY97 will include, in addition to continuation of the activities described above, measurement of edge $T_{e}$ fluctuations and associated turbulent heat transport with Langmuir probes, study of edge plasma conditions in radiative impurity mode (RI-mode) as part of the DIII-D/TEXTOR RI-mode collaboration, coordination of Divertor Material Evaluation Studies (DiMES) experiments (supported by GA), and development of possible new diagnostics for fast radiated power profile measurement during disruption thermal quenches and for fast detection of synchrotron radiation from runaway electrons. To support this expanded program, staffing will be increased to four scientists on-site $100 \%$ at GA, plus additional scientific support from staff based primarily at UCSD.

\subsection{INTERNATIONAL COOPERATION}

The DIII-D international collaboration program continues to provide a broad source of innovative ideas and opportunities which support the DIII-D research program. Collaborations were carried out with JT-60U and JFT-2M in Japan, JET in England, TEXTOR in Germany, Tore Supra in France, and several organizations in Russian and China. In addition to the benefits gained from DIII-D staff assignments in these and other laboratories, foreign scientists visiting DIII-D have made significant contributions to DIII-D program goals. A summary of the progress made by DIII-D staff members in support of the international collaboration program is given below. 


\section{JAERI (JAPAN)}

GA scientists participated in an exchange at JT-60U, working in the area of NCS, High Confinement, and neutral beam current drive. Lang Lao collaborated on using EFIT in NCS configurations. Tony Taylor participated in NCS and High-Q deuterium/tritium (DT) equivalent discharges. Cary Forest worked on non-inductive current drive with high energy negative ion neutral beams.

A successful experiment using the GA designed "Combline" antenna was carried out on the Japan Atomic Energy Research Institute (JAERI) JFT-2M tokamak. This antenna allows better coupling to the plasma over a wide range of plasma parameters.

Dr. N. Isei from JT-60U completed his one year stay at DIII-D. He worked in the area of disruptions, investigating the possibility of using electron cyclotron emission diagnostics for analyzing disruptions. Dr. Y. Koide from JAERI started a long-term exchange on the CER diagnostic.

\section{JET (ENGLAND)}

JET is a large European tokamak approximately twice the size and magnetic field strength of DIII-D. Our collaboration with JET is one of the largest of our international collaborations. This year, several DIII-D scientists from GA, LLNL, and ORNL collaborated at JET in a two part exchange on NCS type high performance tokamak discharges. The results from this series of experiments were very successful. Ion temperatures of about $30 \mathrm{keV}$ and electron temperatures of about $16 \mathrm{keV}$ were obtained. These results represent some of the highest fusion parameters attained in deuterium plasmas on JET. This exchange was a complement to a series of experiments which were performed on DIII-D with participation by JET scientists.

Bill Heidbrink, a DIII-D scientist from the University of California at Irvine, was at JET for five months studying the behavior of Alfven Eigenmodes. These modes, which are waves in the plasma, can cause deleterious effects on ion confinement. Another significant scientific collaboration is the investigation of dimensionally similar discharges on the two devices. These are discharges having the same scaled (physics) parameters, but different physical (engineering) parameters. 


\section{Tore Supra (FRANCE)}

Dr. E. Joffrin came to DIII-D for a three month exchange on electron cyclotron heating (ECH) in DIII-D. Tore Supra is installing an ECH system which has many similar features to the one being developed on DIII-D.

\section{TEXTOR (GERMANY)}

A new operational mode for high tokamak confinement is being pursued on TEXTOR. This high confinement mode involves using a layer of highly radiating plasma on the outer edge of the plasma. For reasons which are not completely understood, this improves the central confinement. DIII-D plans joint experiments with TEXTOR in an attempt to understand this phenomenon.

\section{RUSSIA}

DIII-D has an exchange program with the TRINITI lab at Troitsk, near Moscow. The main topics are: materials for plasma facing components, divertor spectroscopy, and use of the Russiandeveloped DINA code for modeling dynamic plasma behavior.

GA is contracting with Moscow State University to support Russian theorists to perform theoretical analyses of plasma physics problems of relevance to understanding the performance of DIII-D. Included among these are transport and MHD stability modeling of DIII-D, modeling of current drive techniques, $\mathrm{L}-\mathrm{H}$-mode theories and studies of divertor transport. Several of these theorists visited GA in 1996 for discussions with their GA colleagues.

Dr. Trukhin from Kurchatov obtained electron temperature measurements in the DIII-D plasma center during the ECH experiments this year. He used the x-ray spectrometer, purchased from his institute. This instrument was the main diagnostic for central electron temperature measurements during ECH.

Two scientists from the Kurchatov Institute were at GA to be trained in the analysis of CER data. They will remotely analyze the CER data from DIII-D using the virtual memory system (VMS) Alpha workstation which will be sent to the Kurchatov Institute. This work station has been set up with a link to the GA fusion VAX cluster through the internet, to simulate the situation which will exist when it is in Russia. 


\section{NETHERLANDS}

Dr. Joop Konings from F.O.M. in the Netherlands completed a two year exchange working with DIII-D. His primary interest was performing transport modeling of DIII-D plasmas.

\section{CHINA}

This year we had four scientists from Southwestern Institute of Physics and the Institute of Plasma Physics, Academia Sinica, for six month exchanges and a few others for shorter exchanges. These exchanges have concentrated in the area of the Thomson scattering diagnostic, the CER diagnostic, and electron cyclotron range of frequencies systems. 


\section{FY96 PUBLICATIONS}

Allen, S.L., A.S. Bozek, N.H. Brooks, D.A. Buchenauer, K.H. Burrell, T.N. Carlstrom, J.W. Cuthbertson, J.C. DeBoo, R. Ellis, J.C. Evans, T.E. Evans, "Recent DIII-D Divertor Research," in Proc. 22nd European Conference on Controlled Fusion and Plasma Physics, July 3-7, 1995, Bournemouth, United Kingdom, to be published (European Physical Society, Petit-Lancy, Switzerland, 1995); and General Atomics Report GA-A22091 (1995).

Allen, S.L., M.E. Fenstermacher, D.N. Hill, C.J. Lasnier, W.H. Meyer, G.D. Porter, R.D. Wood, A.W. Leonard, M.A. Mahdavi, T.W. Petrie, W.P. West, R. Maingi, M.R. Wade, D.G. Whyte, "Divertor Plasma Parameters During Radiative Divertor Operation on DII-D," Bull. Am. Phys. Soc. 41, 1533 (1996) Abstract.

Allen, S.L., D.N. Hill, T.N. Carlstrom, D.G. Nilson, R.E. Stockdale, C.-L. Hsieh, T.W. Petrie, A.W. Leonard, D. Ryutov, G.D. Porter, R. Maingi, M.R. Wade, R. Cohen, W. Nevins, M.E. Fenstermacher, R.D. Wood, C.J. Lasnier, W.P. West, M.D. Brown, "Measurements of Electron Temperature and Density With DTS in Radiative Divertor Discharges on DIII-D," in Proc. 12th Int. Conf. on Plasma Surface Interactions in Contr. Fusion Devices, May 2024, 1996, Saint Raphael, France, to be published; and General Atomics Report GA-A22357 (1996).

Anderson, P.M., "Scientific Basis and Engineering Design to Accommodate Disruption and Halo Current Loads for the DIII-D Tokamak," in Proc. 19th Symp. on Fusion Technology, September 16-20, 1996, Lisbon, Portugal, to be published; and General Atomics Report GA-A22451 (1996).

Anderson, P.M., "Tokamak Physics Experiment Divertor Design," in Proc. 16th IEEE/NPS Symp. on Fusion Engineering, September 30-October 5, 1995, Champaign, Illinois, Vol. 2, p. 1262 (Institute of Electrical and Electronics Engineers, Inc., Piscataway, New Jersey, 1996).

Austin, M.E., R.F. Ellis, J.L. Doane, R.A. James, "Improved Operation of the Michelson Interferometer ECE Diagnostic on DIII-D," presented at 11th Top. Conf. on High Temperature Plasma Diagnostics, May 12-16, 1996, Monterey, California, to be published in Rev. Sci. Instrum.; and General Atomics Report GA-A22330 (1996).

Baggest, D.S., D.A. Rothweil, S. Pang, M.L. Walker, A. Nerem, "Improving Plasma Shaping Accuracy Through Consolidation of Control Model Maintenance, Diagnostic Calibration, and Hardware Change Control," in Proc. 16th IEEE/NPS Symp. on Fusion Engineering, September 30-October 5, 1995, Champaign, Illinois, Vol. 2, p. 862 (Institute of Electrical and Electronics Engineers, Inc., Piscataway, New Jersey, 1996). 
Baker, D.R., "Density Profile Consistency and Particle Pinch Velocities in DIII-D," Bull. Am. Phys. Soc. 41, 1572 (1996) Abstract.

Baker, D.R., "Modeling of the Recycling Particle Flux and Electron Particle Transport in the DIII-D Tokamak," in Proc. 12th Int. Conf. on Plasma Surface Interactions in Contr. Fusion Devices, May 20-24, 1996, Saint Raphael, France, to be published; and General Atomics Report GA-A22341 (1996).

Baylor, L.R., T.C. Jernigan, M.R. Wade, R. Maingi, R.J. Groebner, M.A. Mahdavi, "Transport in High Density Pellet Fueled DIII-D Discharges,” Bull. Am. Phys. Soc. 41, 1573 (1996) Abstract.

Blue, Anne P., "San Diego Science Alliance Education Outreach Activities," Bull. Am. Phys. Soc. 41, 1455 (1996) Abstract.

Bozek, A.S., C.B. Baxi, J.V. Del Bene, G.J. Laughon, E.E. Reis, H.D. Shatoff, and J.P. Smith, "Engineering Design of Cryocondensation Pumps for the DIII-D Radiative Divertor Program," in Proc. 16th IEEE/NPS Symp. on Fusion Engineering, September 30October 5, 1995, Champaign, Illinois, Vol. 2, p. 898 (Institute of Electrical and Electronics Engineers, Inc., Piscataway, New Jersey, 1996).

Broesch, J.D., "Real Time Software for the Control and Monitoring of DIII-D System Interlocks," in Proc. 19th Symp. on Fusion Technology, September 16-20, 1996, Lisbon, Portugal, to be published; and General Atomics Report GA-A22455 (1996).

Broesch, J.D., E.J. Strait, R.T. Snider, M.L. Walker, “A Digital Long Pulse Integrator," in Proc. 16th IEEE/NPS Symp. on Fusion Engineering, September 30-October 5, 1995, Champaign, Illinois, Vol. 1, p. 365 (Institute of Electrical and Electronics Engineers, Inc., Piscataway, New Jersey, 1996).

Brooks, N.H., D. Fehling, D.L. Hillis, C.C. Klepper, N.N. Naumenko, N. Tugarinov, D.G. Whyte, "Visible Spectroscopy in the DIII-D Divertor," presented at 11th Top. Conf. on High Temperature Plasma Diagnostics, May 12-16, 1996, Monterey, California, to be published in Rev. Sci. Instrum.; and General Atomics Report GA-A22352 (1996).

Brooks, N.H., R.C. Isler, G.R. McKee, S. Tugarinov, "Ion Temperature Measurements in the DII-D Divertor," Bull. Am. Phys. Soc. 41, 1430 (1996) Abstract.

Burrell, K.H., " $\mathrm{E} \times \mathrm{B}$ and Magnetic Shear Effects on Turbulence and Transport in Magnetic Confinement Devices," Bull. Am. Phys. Soc. 41, 1418 (1996) Invited Abstract.

Callis, R.W., "Status of the DIII-D $110 \mathrm{GHz}$ ECH System," in Proc. 12th Top. Mtg. on Technology Fusion Energy, June 16-20, 1996, Reno, Nevada, to be published; and General Atomics Report GA-A22378 (1996).

Callis, R.W., W.P. Cary, R.C. O'Neill, J.C. Allen, T.E. Harris, J.S. deGrassie, S.W. Ferguson, F.W. Baity, G.C. Barber, J.F. Tooker, D.A. Phelps, "Upgrade of the DIII-D RF Systems," in Proc. 16th IEEE/NPS Symp. on Fusion Engineering, September 30October 5, 1995, Champaign, Illinois, Vol. 1, p. 812 (Institute of Electrical and Electronics Engineers, Inc., Piscataway, New Jersey, 1996). 
Carlstrom, T.N., "Transition Physics and Scaling Overview," in Plasma Phys. Control. Fusion 38, 1149 (1996).

Carlstrom, T.N., D.J. Campbell, J.G. Cordey, J.C. DeBoo, R.J. Groebner, E. Righi, G. Sailbene, D.P. Schissel, D. Start, K. Thomsen, "JET/DIII-D Size Scaling of the H-Mode Power Threshold," in Plasma Phys. Control. Fusion 38, 1231 (1996).

Carlstrom, T.N. and R.J. Groebner, "Study of the Conditions for Spontaneous H-mode Transitions in DIII-D," in Phys. Plasmas 3 (5), 1867 (1996).

Carlstrom, T.N., R.J. Groebner, D.M. Thomas, K.H. Burrell, L.W. Owen, B.A. Carreras, "L-H Power Threshold and Transition Studies in DIII-D," Bull. Am. Phys. Soc. 41, 1534 (1996) Abstract.

Carlstrom, T.N., C.-L. Hsieh, R.E. Stockdale, D.G. Nilson, and D.N. Hill, "Initial Operation of the Divertor Thomson Scattering Diagnostic on DIII-D," presented at 11th Top. Conf. on High Temperature Plasma Diagnostics, May 12-16, 1996, Monterey, California, to be published in Rev. Sci. Instrum.; and General Atomics Report GA-A22337 (1996).

Carolipio, E.M., W.W. Heidbrink, "Array of Neutral Particle Analyzers at DIII-D," presented at 11 th Top. Conf. on High Temperature Plasma Diagnostics, May 12-16, 1996, Monterey, California, to be published in Rev. Sci. Instrum.; and General Atomics Report GA-A22358 (1996).

Carolipio, E.M., W.W. Heidbrink, A. Jaun, M.S. Chu, R.B. White, C.Z. Cheng, G.Y. Fu, "Simulations of Energetic-Ion Losses During TAE Activity in DIII-D," Bull. Am. Phys. Soc. 41, 1570 (1996) Abstract.

Carreras, B.A., L. Owen, R. Maingi, P. Mioduszewski, T.N. Carlstrom, R.J. Groebner, "Studies of Edge Neutral Density Distributions in L- and H-mode Discharges," Bull. Am. Phys. Soc. 41, 1573 (1996) Abstract.

Cary, W.P., F.W. Baity, G.C. Barber, R.W. Callis, J.S. deGrassie, S.W. Ferguson, T.E. Harris, R.C. O'Neill, R.I. Pinsker, "4 MW Upgrade to DIII-D FWCD System: System Commissioning and Initial Operation," in Proc. 16th IEEE/NPS Symp. on Fusion Engineering, September 30-October 5, 1995, Champaign, Illinois, Vol. 1, p. 834 (Institute of Electrical and Electronics Engineers, Inc., Piscataway, New Jersey, 1996).

Casper, T.A., M.C. Spang, J. Crotinger, L.D. Pearlstein, B.W. Stallard, T.S. Taylor, "Corsica Time-Dependent Modeling of DIII-D Discharges," Bull. Am. Phys. Soc. 41, 1571 (1996) Abstract.

Chan, V.S., for the DIII-D Team, "DIII-D Tokamak Concept Improvement Research," in Proc. 16th Int. Conf. on Plasma Phys. and Controlled Nucl. Fusion Research, October 7-11, 1996, Montreal, Canada (International Atomic Energy Agency, Vienna, to be published); and General Atomics Report GA-A22471 (1996). 
Chiu, H.K., "Calculations of Material Activation in Disruption Generated Run-Away Electrons in ITER,” Bull. Am. Phys. Soc. 41, 1436 (1996) Abstract.

Chiu, S.C., V.S. Chan, R.W. Harvey, M.N. Rosenbluth, "Bounce-Averaged Fokker-Planck Simulation of Runaway Avalanche from Secondary Knock-on Production," Bull. Am. Phys. Soc. 41, 1435 (1996) Abstract.

Chiu, S.C., J.S. deGrassie, R.W. Harvey, V.S. Chan, Y.R. Lin-Liu, R.D. Stambaugh, H. Ikezi, T.K. Mau, W.W. Heidbrink, "Interaction of Fast Waves with Ions," in Proc. 11th Top. Conf. on Radio Frequency Power in Plasmas, May 17-19, 1995, Palm Springs, California, to be published; and General Atomics Report GA-A22062 (1995).

Chu, M.S., J.M. Greene, L.L. Lao, A. Bondeson, O. Sauter, B.W. Rice, E.J. Strait, T.S. Taylor, A.D. Turnbull, "Resistive Instabilities in Advanced Negative Central Shear Tokamaks With Peaked Pressure Profiles," in Proc. 23rd European Conf. on Contr. Fusion and Plasma Phys., June 24-28, 1996, Kiev, Ukraine, Vol. 20C, Part I, p. 215 (European Physical Society, Petit-Lancy, Switzerland, 1996).

Chu, M.S., Y.R. Lin-Liu, R.L. Miller, F.L. Waelbroeck, "Beta Optimization in High Temperature Steady-State Tokamaks,” Bull. Am. Phys. Soc. 41, 1398 (1996) Abstract.

Coda, S., M. Porkolab, K.H. Burrell, "Edge Turbulence Correlation Measurements by PhaseContrast Imaging in DIII-D," Bull. Am. Phys. Soc. 41, 1574 (1996) Abstract.

Coda, S., M. Porkolab, K.H. Burrell, "Edge Turbulence Measurements During the L- to H-Mode Transition by Phase Contrast Imaging on DIII-D," in Proc. 22nd European Conference on Controlled Fusion and Plasma Physics, July 3-7, 1995, Bournemouth, United Kingdom, Vol. 19C, Part 1, p. I-297 (European Physical Society, Petit-Lancy, Switzerland, 1995); and General Atomics Report GA-A22086 (1995).

Danielson, C.A., "Fusion Science Education Outreach," Bull. Am. Phys. Soc. 41, 1456 (1996) Abstract.

DeBoo, J.C., "Global Energy Confinement Scaling, Uncertainties, and Projections for ITER," Bull. Am. Phys. Soc. 41, 1414 (1996) Invited Abstract.

DeBoo, J.C., C.M. Greenfield, D.P. Schissel, K.H. Burrell, R.J. Groebner, L.L. Lao, E.J. Strait, E.A. Lazarus, M. Murakami, B.W. Rice, B.W. Stallard, G.A. Navratil, "Profile Evolution in Enhanced Performance Discharges in DIII-D," Bull. Am. Phys. Soc. 41, 1571 (1996) Abstract.

deGrassie, J.S., H. Ikezi, L.L. Lao, Y.R. Lin-Liu, C.C. Petty, R.I. Pinsker, R. Prater, F.W. Baity, M. Murakami, "Fast Wave Electron Heating in ELMing $\mathrm{H}$-mode Discharges," Bull. Am. Phys. Soc. 41, 1429 (1996) Abstract.

deGrassie, J.S., C.C. Petty, R.I. Pinsker, C.B. Forest, H. Ikezi, R. Prater, F.W. Baity, R.W. Callis, W.P. Cary, S.C. Chiu, E.J. Doyle, "Fast Wave Current Drive on DIII-D," in Proc. 11 th Top. Con. on Radio Frequency Power in Plasmas, May 17-19, 1995, Palm Springs, California, p. 173 (1996). 
Doyle, E.J., K.H. Burrell, T.N. Carlstrom, S. Coda, J.C. DeBoo, R. Durst, R.J. Fonck, P. Gohil, C.M. Greenfield, R.J. Groebner, J. Kim, K.W. Kim, R.J. La Haye, L.L. Lao, E.A. Lazarus, R.A. Moyer, G.A. Navratil, T.H. Osborne, W.A. Peebles, C.L. Rettig, T.L. Rhodes, B.W. Rice, D.P. Schissel, G.M. Staebler, E.J. Strait, T.S. Taylor, D.M. Thomas, R.E. Waltz, "Physics of Turbulence Control and Transport Barrier Formation in DII-D," in Proc. 16th Int. Conf. on Plasma Phys. and Controlled Nucl. Fusion Research, October 711, 1996, Montreal, Canada (International Atomic Energy Agency, Vienna, to be published); and General Atomics Report GA-A22487 (1996).

Doyle, E.J., W.A. Peebles, C.L. Rettig, T.L. Rhodes, R.A. Moyer, R.J. Groebner, K.H. Burrell, P. Gohil, R. Maingi, G.D. Porter, J.G. Watkins, "Physics of Very Slow L-H Transitions," Bull. Am. Phys. Soc. 41, 1574 (1996) Abstract.

Evans, T.E., A.G. Kellman, D.A. Humphreys, M.J. Schaffer, P.L. Taylor, D.G. Whyte, T.C. Jernigan, A.W. Hyatt, and R.L. Lee, "Measurements of Non-Axisymmetric Halo Currents With and Without 'Killer' Pellets During Disruptions in the DIII-D Tokamak," in Proc. 12th Int. Conf. on Plasma Surface Interactions in Contr. Fusion Devices, May 20-24, 1996, Saint Raphael, France, to be published; and General Atomics Report GA-A22344 (1996).

Evans, T.E., M.E. Fenstermacher, M.A. Mahdavi, W.H. Meyer, G.D. Porter, G.T. Sager, W.P. West, "Monte-Carlo Impurity Transport Simulations in the Edge of the DIII-D Tokamak Using the MCI Code," in Proc. 10th International Conference on Stellarators, May 22-26, 1995, Madrid, Spain, to be published; and General Atomics Report GA-A22064 (1995).

Evans, T.E., P.L. Taylor, A.G. Kellman, M.J. Schaffer, A.W. Hyatt, D.A. Humphreys, R.L. Lee, P.B. Parks, D.G. Whyte, T.C. Jernigan, S. Luckhardt, J.W. Cuthbertson, J. Zhang, G.W. Jahns, D. Wroblewski, "Recent Physics Results from the DIII-D Disruption Program," Bull. Am. Phys. Soc. 41, 1534 (1996) Abstract.

Fenstermacher, M.E., "Comprehensive 2D Measurements of Radiative Divertor Plasmas in DIII-D," in Proc. 12th Int. Conf. on Plasma Surface Interactions in Contr. Fusion Devices, May 20-24, 1996, Saint Raphael, France, to be published; and General Atomics Report GA-A22347 to be published.

Fenstermacher, M.E., "The Two-Dimensional Structure of Partially Detached Divertor Plasmas in the DIII-D Tokamak," Bull. Am. Phys. Soc. 41, 1560 (1996) Invited Abstract.

Fenstermacher, M.E., S.L. Allen, D.N. Hill, A.W. Leonard, C.J. Lasnier, T.W. Petrie, R.D. Wood, N.H. Brooks, D.A. Buchenauer, T.N. Carlstrom, J.W. Cuthbertson, E.J. Doyle, T.E. Evans, P.-M. Garbet, R.W. Harvey, A.W. Hyatt, R.C. Isler, G.L. Jackson, R.A. James, R.A. Jong, C.C. Klepper, M.A. Mahdavi, R. Maingi, W.H. Meyer, R.A. Moyer, D.G. Nilson, G.D. Porter, T.E. Rhodes, M.J. Schaffer, G.M. Staebler, R.D. Stambaugh, R.E. Stockdale, D.M. Thomas, S. Tugarinov, M.R. Wade, J.G. Watkins, W.P. West, D.G. Whyte, "A Comprehensive 2-D Divertor Data Set for DIII-D for Edge Theory Validation," Contrib. to Plasma Phys. 36, 127 (1996). 
Ferguson, S.W., R.W. Callis, W.P. Cary, T.E. Harris, and J.C. Allen, "RF Power Diagnostics and Control on the DIII-D, $4 \mathrm{MW} 30-120 \mathrm{MHz}$ Fast Wave Current Drive System (FWCD)," in Proc. 16th IEEE/NPS Symp. on Fusion Engineering, September 30October 5, 1995, Champaign, Illinois, Vol. 2, p. 866 (Institute of Electrical and Electronics Engineers, Inc., Piscataway, New Jersey, 1996).

Ferguson, S.W., R.W. Callis, W.P. Cary, D.A. Phelps, D. Ponce, F.W. Baity, and G. Barber, "RF High Voltage Performance of RF Transmission Line Components on the DIII-D Fast Wave Current Drive (FWCD) System," in Proc. 16th IEEE/NPS Symp. on Fusion Engineering, September 30-October 5, 1995, Champaign, Illinois, Vol. 1, p. 837 (Institute of Electrical and Electronics Engineers, Inc., Piscataway, New Jersey, 1996).

Ferron, J.R., B. Penaflor, M.L. Walker, J. Moller, D. Butner, "A Flexible Software Architecture for Tokamak Discharge Control Systems," in Proc. 16th IEEE/NPS Symp. on Fusion Engineering, September 30-October 5, 1995, Champaign, Illinois, Vol. 2, p. 870 (Institute of Electrical and Electronics Engineers, Inc., Piscataway, New Jersey, 1996).

Ferron, J.R., M.L. Walker, B. Penaflor, H.E. St. John, "Real Time Equilibrium Reconstruction for Control of the Discharge in the DIII-D Tokamak," Bull. Am. Phys. Soc. 41, 1569 (1996) Abstract.

Forest, C.B., S.C. Chiu, J.S. deGrassie, H. Ikezi, C.C. Petty, R.I. Pinsker, R. Prater, D.B. Batchelor, F.W. Baity, E.F. Jaeger, M. Murakami, J.H. Lee, M. Porkolab, "Application of Fast Wave Current Drive to Discharges with Negative Central Shear," Bull. Am. Phys. Soc. 41, 1387 (1996) Abstract.

Forest, C.B., C.C. Petty, F.W. Baity, S.C. Chiu, J.S. deGrassie, R.J. Groebner, H. Ikezi, E.F. Jaeger, K. Kupfer, M. Murakami, R.I. Pinsker, "Experimentally Determined Profiles of Fast Wave Current Drive on DIII-D," in Proc. 11th Top. Con. on Radio Frequency Power in Plasmas, May 17-19, 1995, Palm Springs, California, to be published; and General Atomics Report GA-A22048 (1995).

Forest, C.B., C.C. Petty, K.H. Burrell, J.S. deGrassie, A.W. Hyatt, R.I. Pinsker, M. Porkolab, M. Murakami, R. Prater, B.W. Rice, E.J. Strait, "Fast Wave Heating and Current Drive in Tokamak Plasmas with Negative Central Shear," in Proc. 23rd European Conf. on Contr. Fusion and Plasma Phys., June 24-28, 1996, Kiev, Ukraine, Vol. 20C, Part II, p. 910 (European Physical Society, Petit-Lancy, Switzerland, 1996).

Freeman, R.L., "Applications of High Power Millimeter Waves in the DIII-D Fusion Program," in Proc. Int. Conf. on Millimeter and Submillimeter Waves and Applications III, August 59, 1996, Denver, Colorado, Vol. 2842, p. 11 (SPIE - The International Society for Optical Engineering, Bellingham, Washington, 1996).

Galkin, S.A., A.A. Ivanov, S.Yu. Medvedev, Yu.Yu. Poshekhonov, D.A. Humphreys, "Comparison of Tokamak Axisymmetric Mode Growth Rates for Linear MHD and Equilibrium Evolution Approaches," Bull. Am. Phys. Soc. 41, 1375 (1996) Abstract.

Gohil, P., K.H. Burrell, T.H. Osborne, A.B. Hassam, "Experimental Evaluations of Theoretical Predictions for Plasma Rotation at the L-H Transition in DIII-D," Bull. Am. Phys. Soc. 41, 1573 (1996) Abstract. 
Gootgeld, A.M., "Cooling Water Conditioning \& Quality Control For Tokamaks," in Proc. 16th IEEE/NPS Symp. on Fusion Engineering, September 30-October 5, 1995, Champaign, Illinois, Vol. 1, p. 825 (Institute of Electrical and Electronics Engineers, Inc., Piscataway, New Jersey, 1996).

Greene, J.M., O.N. Boratav, "Evidence for the Development of Singularities in Euler Flow," Physica D 91, 1 (1996).

Greenfield, C.M., "Transport and Performance in DIII-D Discharges with Weak or Negative Central Magnetic Shear," Bull. Am. Phys. Soc. 41, 1443 (1996) Invited Abstract.

Greenfield, C.M., D.P. Schissel, B.W. Stallard, E.A. Lazarus, G.A. Navratil, R.V. Budny, K.H. Burrell, T.A. Casper, J.C. DeBoo, E.J. Doyle, R. Durst, R.J. Fonck, R.J. Groebner, L.L. Lao, D.C. McCune, M. Murakami, C.C. Petty, C.L. Rettig, B.W. Rice, T.L. Rhodes, H.E. St. John, T.S. Taylor, K.L. Tritz, M.R. Wade, D.G. Whyte, "Transport in High Performance Weak and Negative Central Shear Discharges in DIII-D," in Proc. 23rd European Conf. on Contr. Fusion and Plasma Phys., June 24-28, 1996, Kiev, Ukraine, Vol. 20C, Part I, p. 291 (European Physical Society, Petit-Lancy, Switzerland, 1996).

Groebner, R.J., T.N. Carlstrom, "Parameterization of Edge Profiles for $\mathrm{H}$-mode Studies," Bull. Am. Phys. Soc. 41, 1573 (1996) Abstract.

Groebner, R.J., T.N. Carlstrom, K.H. Burrell, S. Coda, E.J. Doyle, P. Gohil, K.W. Kim, Q. Peng, R. Maingi, R.A. Moyer, C.L. Rettig, T.L. Rhodes, D.P. Schissel, G.M. Staebler, R.D. Stambaugh, D.M. Thomas, J.G. Watkins, "Study of H-Mode Threshold Conditions in DIII-D," in Proc. 16th Int. Conf. on Plasma Phys. and Controlled Nucl. Fusion Research, October 7-11, 1996, Montreal, Canada (International Atomic Energy Agency, Vienna, to be published); and General Atomics Report GA-A22443 (1996).

Harris, J.J., J.D. Broesch, R.M. Coon, "A Combined PLC and CPU Approach to Multiprocessor Control," in Proc. 16th IEEE/NPS Symp. on Fusion Engineering, September 30-October 5, 1995, Champaign, Illinois, Vol. 2, p. 874 (Institute of Electrical and Electronics Engineers, Inc., Piscataway, New Jersey, 1996).

Harris, T.E., J.C. Allen, W.P. Cary, S.W. Ferguson, C.C. Petty, and R.I. Pinsker, "System Control and Data Acquisition of the Two New FWCD RF Systems at DIII-D," in Proc. 16th IEEE/NPS Symp. on Fusion Engineering, September 30-October 5, 1995, Champaign, Illinois, Vol. 2, p. 878 (Institute of Electrical and Electronics Engineers, Inc., Piscataway, New Jersey, 1996).

Harvey, R.W., V.S. Chan, S.C. Chiu, M.N. Rosenbluth, "Runaway Electron Distribution Calculation with the CQL3D Fokker-Planck Code," Bull. Am. Phys. Soc. 41, 1436 (1996) Abstract.

Heidbrink, W.W., "Beam-Driven Chirping Instability in DIII-D," in Plasma Physics and Controlled Fusion 37, 937 (1995). 
Henline, P.A., "Improved Operating Scenarios of the DIII-D Tokamak as a Result of the Addition of UNIX Computer Systems," in Proc. 16th IEEE/NPS Symp. on Fusion Engineering, September 30-October 5, 1995, Champaign, Illinois, Vol. 2, p. 895 (Institute of Electrical and Electronics Engineers, Inc., Piscataway, New Jersey, 1996).

Hill, D.N., S.L. Allen, R.D. Wood, T.W. Petrie, A.W. Leonard, W.P. West, M. Wade, R. Isler, R. Maingi, "Results of Recent Radiative Divertor Experiments in DIII-D," Bull. Am. Phys. Soc. 41, 1430 (1996) Abstract.

Hinton, F.L., "Nonlocal Collisional Transport in a Magnetized Plasma," Bull. Am. Phys. Soc. 41, 1394 (1996) Abstract.

Hodapp, T.R., J.D. Broesch, J.A. Leuer, R.T. Snider, E.J. Strait, "Magnetic Diagnostics for Future Tokamaks," in Proc. 16th IEEE/NPS Symp. on Fusion Engineering, September 30October 5, 1995, Champaign, Illinois, Vol. 2, p. 918 (Institute of Electrical and Electronics Engineers, Inc., Piscataway, New Jersey, 1996).

Hollerbach, M.A., J.P. Smith, C.B. Baxi, A.S. Bozek, E. Chin, R.D. Phelps, K.M. Redler, and E.E. Reis, "Design and Analysis of the DIII-D Radiative Divertor Water-Cooled Structures," in Proc. 16th IEEE/NPS Symp. on Fusion Engineering, September 30-October 5, 1995, Champaign, Illinois, Vol. 1, p. 817 (Institute of Electrical and Electronics Engineers, Inc., Piscataway, New Jersey, 1996).

Humphreys, D.A., A.G. Kellman, T.E. Evans, M.J. Schaffer, "Predictive Modeling of Axisymmetric Disruption Halo Currents," Bull. Am. Phys. Soc. 41, 1432 (1996) Abstract.

Hyatt, A.W., R.L. Lee, J.W. Cuthbertson, D.A. Humphreys, A.G. Kellman, C.J. Lasnier, P.L. Taylor, and The DIII-D Team, "Magnetic and Thermal Energy FLow During Disrution in DIII-D," in Proc. 23rd European Conf. on Contr. Fusion and Plasma Phys., June 24-28, 1996, Kiev, Ukraine, Vol. 20C, Part I, p. 287 (European Physical Society, Petit-Lancy, Switzerland, 1996).

Hyatt, A.W., R.L. Lee, D.A. Humphreys, A.G. Kellman, P.L. Taylor, and The DIII-D Team, J.W. Cuthbertson, "Magnetic and Thermal Energy Flow During Disruptions in DIII-D," Bull. Am. Phys. Soc. 41, 1433 (1996) Abstract.

Ikezi, H., J.S. deGrassie, R.I. Pinsker, R.T. Snider, "Plasma Mass Density, Species Mix and Fluctuation Diagnostics Using Fast Alfven Wave," presented at 11th Top. Conf. on High Temperature Plasma Diagnostics, May 12-16, 1996, Monterey, California, to be published in Rev. Sci. Instrum.; and General Atomics Report GA-A22340 (1996).

Ikezi, H., D.A. Phelps, "Traveling Wave Antenna for Fast Wave Heating and Current Drive in Tokamaks," to be published in Fusion Tech.; and General Atomics Report GA-A21993 (1995).

Ikezi, H., R.I. Pinsker, S.C. Chiu, J.S. deGrassie, "Experimental Studies of Fast Wave Propagation in DIII-D," in Proc. 11th Top. Con. on Radio Frequency Power in Plasmas, May 1719, 1995, Palm Springs, California, to be published; and General Atomics Report GA-A22057 (1995). 
Ikezi, H., R.I. Pinsker, S.C. Chiu, J.S. deGrassie, "Fast Wave Propagation Studies in the DIII-D Tokamak," in Phys. Plasmas 3 (6), 2306 (1996).

Isler, R.C., C.C. Klepper, R.D. Wood, M.E. Fenstermacher, A.W. Leonard, "Spectroscopic Characterization of the DIII-D Divertor," Bull. Am. Phys. Soc. 41, 1430 (1996) Abstract.

Jackson, G.L., E.A. Lazarus, G.A. Navratil, R.J. Bastasz, N.H. Brooks, D.T. Garnier, K.L. Holtrop, J.C. Phillips, E.S. Marmar, T.S. Taylor, D.M. Thomas, W.R. Wampler, D.G. Whyte, W.P. West, "Enhanced Performance in the DIII-D Tokamak with Lithium Wall Conditioning," in Proc. 12th Int. Conf. on Plasma Surface Interactions in Contr. Fusion Devices, May 20-24, 1996, Saint Raphael, France, to be published; and General Atomics Report GA-A22361 (1996).

Jackson, G.L., G.M. Staebler, S.L. Allen, N.H. Brooks, T.E. Evans, J.R. Ferron, A.W. Leonard, R. Maingi, T.W. Petrie, M.J. Schaffer, R.D. Wood, W.P. West, D.G. Whyte, "Impurity Feedback Control for Enhanced Divertor and Edge Radiation in DIII-D Discharges," in Proc. 12th Int. Conf. on Plasma Surface Interactions in Contr. Fusion Devices, May 20-24, 1996, Saint Raphael, France, to be published; and General Atomics Report GA-A22345 (1996).

Jackson, G.L., G.M. Staebler, A.W. Leonard, T.W. Petrie, M.J. Schaffer, W.P. West, "CDH-mode and Highly Radiative Discharges in the DIII-D Tokamak," Bull. Am. Phys. Soc. 41, 1430 (1996) Abstract.

Jaeger, E.F., M. Murakami, L.A. Berry, D.B. Batchelor, M.D. Carter, F.W. Baity, S.C. Chiu, J.S. deGrassie, C.B. Forest, C.C. Petty, R.I. Pinsker, R. Prater, "Absorption of Fast Wave Power by Energetic Ions During FWCD in DIII-D," Bull. Am. Phys. Soc. 41, 1534 (1996) Abstract.

James, R.A., C.C. Petty, R.W. Harvey, "A Comparison of Fundamental and Second Harmonic Inside Launch ECCD in the Presence of a dc Electric Field," in Proc. 9th Joint Workshop on Electron Cyclotron Emission and Electron Cyclotron Heating Conference, January 23-26, 1995, Borrego Springs, California, p. 75 (World Scientific Publishing Co., Singapore, Japan 1995).

Jensen, T.H., K.H. Finken, C. Ren, "Nonlinear Tearing," Bull. Am. Phys. Soc. 41, 1398 (1996) Abstract.

Jensen, T.H., R.J. La Haye, A.W. Hyatt, "A Nonlinear Model for the Singular Surface Response," in Phys. Plasmas 3 (5) 1524 (1996).

Jensen, T.H., R.L. Miller, Y.R. Lin-Liu, "Pressure Driven Tokamaks," Phys. Plasmas 3, 1656 (1996).

Jernigan, T.C., L.R. Baylor, S.K. Combs, S.L. Milora, M.R. Wade, T.E. Evans, D.A. Humphreys, M.A. Mahdavi, J.C. Phillips, R.T. Snider, "Pellet Injection on DIII-D," Bull. Am. Phys. Soc. 41, 1572 (1996) Abstract. 
Johnson, W.R., J.P. Smith, R.D. Stambaugh, "Production and Fabrication of Vanadium Alloys for the Radiative Divertor Program of DIII-D," General Atomics Report GA-A22426 (1996).

Kellman, A.G., J.W. Cuthbertson, T.E. Evans, D.A. Humphreys, A.W. Hyatt, G.L. Jahns, T.C. Jernigan, C.J. Lasnier, R.L. Lee, J.A. Leuer, S. Luckhardt, M.J. Schaffer, P.L Taylor, D.G. Whyte, D. Wroblewski, J. Zhang, "Disruption Studies in DIII-D," in Proc. 16th Int. Conf. on Plasma Phys. and Controlled Nucl. Fusion Research, October 7-11, 1996, Montreal, Canada (International Atomic Energy Agency, Vienna, to be published); and General Atomics Report GA-A22476 (1996).

Kellman, A.G., for the DIII-D Team, "Recent Results from the DIII-D Tokamak," in Proc. 16th IEEE/NPS Symp. on Fusion Engineering, September 30-October 5, 1995, Champaign, Illinois, Vol. 1, p. 792 (Institute of Electrical and Electronics Engineers, Inc., Piscataway, New Jersey, 1996).

Kessler, D.N., R.-M. Hong, S.P. Riggs, “A New Technique to Measure the Neutralizer Cell Gas Line Density Applied to a DIII-D Neutral Beamline," in Proc. 16th IEEE/NPS Symp. on Fusion Engineering, September 30-October 5, 1995, Champaign, Illinois, Vol. 1, p. 840 (Institute of Electrical and Electronics Engineers, Inc., Piscataway, New Jersey, 1996).

Kim, J.S., “A Method of Poloidal Mode Analysis of Tokamak Plasmas," Bull. Am. Phys. Soc. 41, 1571 (1996) Abstract.

Kim, K.W., E.J. Doyle, T.L. Rhodes, W.A. Peebles, C.L. Rettig, N.C. Luhmann, Jr., "High Resolution Reflectometer Edge Density Profile Measurements at L-H Transitions and ELMs," Bull. Am. Phys. Soc. 41, 1574 (1996) Abstract.

La Haye, R.J., J.D. Callen, M.S. Chu, S. Deshpande, T.A. Gianakon, C.C. Hegna, S. Jardin, L.L. Lao, J. Manickam, D.A. Monticello, A. Pletzer, A.J. Reiman, O. Sauter, E.J. Strait, T.S. Taylor, A.D. Turnbull, H.R. Wilson, "Practical Beta Limit in ITER-Shaped Discharges in DIII-D and Its Increase by Higher Collisionality," in Proc. 16th Int. Conf. on Plasma Phys. and Controlled Nucl. Fusion Research, October 7-11, 1996, Montreal, Canada (International Atomic Energy Agency, Vienna, to be published); and General Atomics Report GA-A22427 (1996).

La Haye, R.J., L.L. Lao, E.J. Strait, T.S. Taylor, "High Beta Tokamak Operation in DIII-D Limited at Low Density/Collisionality by Resistive Tearing Modes," to be published in Phys. Rev. Lett.; and General Atomics Report GA-A22229 (1996).

La Haye, R.J., L.L. Lao, E.J. Strait, T.S. Taylor, "Long-Pulse Beta Limit and Neoclassical Tearing in DIII-D," Bull. Am. Phys. Soc. 41, 1388 (1996) Abstract.

La Haye, R.J., J.T. Scoville, "Use of an $M=2, N=1$ Static Error Field Correction Coil, 'The C-Coil,' on DIII-D to Avoid Disruptive Locked Modes," in Proc. 22nd European Conference on Controlled Fusion and Plasma Physics, June 3-7, 1995, Bournemouth, United Kingdom, Vol. 19C, Part IV, p. 53 (European Physical Society, Petit-Lancy, Switzerland, 1995). 
Lao, L.L., K.H. Burrell, T.S. Casper, V.S. Chan, M.S. Chu, J.C. DeBoo, E.J. Doyle, R.D. Durst, C.B. Forest, C.M. Greenfield, R.J. Groebner, F.L. Hinton, Y. Kawano, E.A. Lazarus, Y.R. Lin-Liu, M.E. Mauel, W.H. Meyer, R.L. Miller, G.A. Navritil, T.H. Osborne, Q. Peng, C.L. Rettig, G. Rewoldt, T.L. Rhodes, B.W. Rice, D.P. Schissel, B.W. Stallard, E.J. Strait, W.M. Tang, T.S. Taylor, A.D. Turnbull, R.E. Waltz, and The DIII-D Team, "Rotational and Magnetic Shear Stabilization of Magnetohydrodynamic Modes and Turbulence in DII-D High Performance Discharges," in Phys. Plasmas 5, 1951 (1996).

Lao, L.L., K.H. Burrell, M.S. Chu, J.R. Ferron, A.W. Hyatt, G.L. Jackson, T.C. Luce, R.L. Miller, T.H. Osborne, P.A. Politzer, G.M. Staebler, E.J. Strait, T.S. Taylor, A.D. Turnbull, E.A. Lazarus, G.A. Navratil, G. Rewoldt, B.W. Rice, B.W. Stallard, "Enhanced Stability and Confinement in DIII-D Discharges with Weak Magnetic Shear and Recent Experiments in Crescent/Bean Geometry," Bull. Am. Phys. Soc. 41, 1569 (1996) Abstract.

Lasnier, C.J., M.E. Fenstermacher, G.D. Porter, B.W. Rice, B.W. Stallard, R. Maingi, A.W. Leonard, J.G. Watkins, "Edge Properties of DIII-D High Performance and Extended Duration Pumped NCS Discharges,” Bull. Am. Phys. Soc. 41, 1432 (1996) Abstract.

Lasnier, C.J., R. Maingi, A.W. Leonard, S.L. Allen, D.A. Buchenauer, K.H. Burrell, T.A. Casper, J.W. Cuthbertson, M.E. Fenstermacher, D.N. Hill, R.A. Jong, L.L. Lao, E.A. Lazarus, R.A. Moyer, T.W. Petrie, G.D. Porter, B.W. Rice, B.W. Stallard, T.S. Taylor, J.G. Watkins, "Characteristics of the Scrape-Off Layer in DIII-D High-Performance Negative Central Magnetic Shear Discharges," in Proc. 12th Int. Conf. on Plasma Surface Interactions in Contr. Fusion Devices, May 20-24, 1996, Saint Raphael, France, to be published; and General Atomics Report GA-A22353 (1996).

Lazarus, E.A., G.A. Navratil, C.M. Greenfield, E.J. Strait. M.E. Austin, K.H. Burrell, T.A. Casper, D.R. Baker, J.C. DeBoo, E.J. Doyle, R. Durst, J.R. Ferron, C.B. Forest, P. Gohil, R.J. Groebner, W.W. Heidbrink, R.-M. Hong, A.W. Howald, C.-L. Hsieh, A.W. Hyatt, G.L. Jackson, J. Kim, L.L. Lao, C.J. Lasnier, A.W. Leonard, J. Lohr, R.J. La Haye, R. Maingi, R.L. Miller, M. Murakami, T.H. Osborne, L.J. Perkins, C.C. Petty, C.L. Rettig, T.L. Rhodes, B.W. Rice, S.A. Sabbagh, D.P. Schissel, J.T. Scoville, R.T. Snider, B.W. Stallard, R.D. Stambaugh, H.E. St. John, R.E. Stockdale, P.L. Taylor, T.S. Taylor, D.M. Thomas, A.D. Turnbull, M.R. Wade, R.D. Wood, D.G. Whyte, "Higher Fusion Power Gain With Profile Control in DIII-D Tokamak Plasmas," to be published in Nucl. Fusion; and General Atomics Report GA-A22416 (1996).

Lazarus, E.A., G.A. Navratil, E.J. Strait, C.M. Greenfield, The DIII-D Team, B.W. Rice, "Increasing the Fusion Gain in DIII-D," Bull. Am. Phys. Soc. 41, 1386 (1996) Abstract.

Lazarus, E.A., G.A. Navratil, E.J. Strait, B.W. Rice, J.R. Ferron, C.M. Greenfield, M.E. Austin, D.R. Baker, K.H. Burrell, T.A. Casper, V.S. Chan, J.C. DeBoo, E.J. Doyle, R. Durst, C.B. Forest, P. Gohil, R.J. Groebner, W.W. Heidbrink, R.-M. Hong, W.A. Houlberg, A.W. Howald, C.-L. Hsieh, A.W. Hyatt, G.L. Jackson, J. Kim, R.J. La Haye, L.L. Lao, C.J. Lasnier, A.W. Leonard, J. Lohr, R. Maingi, R.L. Miller, M. Murakami, T.H. Osborne, L.J. Perkins, C.C. Petty, C.L. Rettig, R.L. Rhodes, S.A. Sabbagh, D.P. Schissel, J.T. Scoville, A.C.C. Sips, R.T. Snider, F.X. Sölder, G.M. Staebler, B.W. Stallard, R.D. Stambaugh, H.E. St John, R.E. Stockdale, T.S. Taylor, D.M. Thomas, A.D. Turnbull, M.R. Wade, R.D. Wood, D.G. Whyte, "Stability in High Gain Plasmas in DIII-D," in Proc. 16th Int. Conf. on Plasma Phys. and Controlled Nucl. Fusion Research, October 7-11, 1996, Montreal, Canada (International Atomic Energy Agency, Vienna, to be published); and General Atomics Report GA-A22489 (1996). 
Lee, R.L., "Presentation Stations of the General Atomics Fusion Educational Program," Bull. Am. Phys. Soc. 41, 1456 (1996) Abstract.

Lee, R.L., A.W. Hyatt, A.G. Kellman, P.L. Taylor, C.J. Lasnier, "Heat Flux Asymmetry in Disruptions and VDEs in DIII-D," Bull. Am. Phys. Soc. 41, 1433 (1996) Abstract.

Lee, R.L., C.J. Lasnier, A.W. Hyatt, A.G. Kellman, P.L. Taylor, "Thermal Deposition Analysis During Disruptions on DIII-D Using Infrared Scanners," in Proc. 16th IEEE/NPS Symp. on Fusion Engineering, September 30-October 5, 1995, Champaign, Illinois, Vol. 2, p. 902 (Institute of Electrical and Electronics Engineers, Inc., Piscataway, New Jersey, 1996).

Leonard, A.W., S.L. Allen, M.E. Fenstermacher, D.N. Hill, C.J. Lasnier, W.M. Meyer, T.W. Petrie, J.G. Watkins, R.D. Wood, DIII-D Team, "Radiation Distributions in Detached Divertor Operation on DIII-D," in Proc. 22nd European Conference on Controlled Fusion and Plasma Physics, June 3-7, 1995, Bournemouth, United Kingdom, to be published (European Physical Society, Petit-Lancy, Switzerland, 1995); and General Atomics Report GA-A22068 (1995).

Leonard, A.W., J. Goetz, C. Fuchs, M. Marashek, F. Mast, R. Reichle, "Bolometry for Divertor Characterization and Control," in Proc. The Diagnostics for ITER International Workshop, August 28-September 1, 1995, Varenna, Italy, to be published; and General Atomics Report GA-A22138 (1995).

Leonard, A.W., T.W. Petrie, W.P. West, M.E. Fenstermacher, D.N. Hill, G.D. Porter, R.D. Wood, J.G. Watkins, D.G. Whyte, "Enhanced Distributed Radiation in an Extended Divertor in DIII-D," Bull. Am. Phys. Soc. 41, 1533 (1996) Abstract.

Leonard, A.W., W. Suttrop, T.H. Osborne, T.E. Evans, D.N. Hill, A. Hermann, C.J. Lasnier, D.M. Thomas, J.G. Watkins, W.P. West, M. Weinlich, H. Zohm, "Divertor Heat and Particle Flux D to ELMs in DIII-D and ASDEX-U," in Proc. 12th Int. Conf. on Plasma Surface Interactions in Contr. Fusion Devices, May 20-24, 1996, Saint Raphael, France, to be published; and General Atomics Report GA-A22346 (1996).

Lin-Liu, Y.R., V.S. Chan, S.C. Chiu, "Plasma Rotation in the Presence of Radio Frequency Fields," in Proc. 11th Top. Con. on Radio Frequency Power in Plasmas, May 17-19, 1995, Palm Springs, California, p. 247 (American Institute of Physics, New York 1996).

Lin-Liu, Y.R., R.L. Miller, V.S. Chan, R. Prater, T.S. Taylor, "Stability of Bootstrap CurrentDriven Tokamak Equilibrium on Current Diffusion Time Scale," Bull. Am. Phys. Soc. 41, 1429 (1996) Abstract.

Lohr, John, R.W. Callis, Dan Ponce, J.T. Tooker, L. Popov, Daqing Zhang, "Commissioning of the First Part of the $110 \mathrm{GHz}$ ECH System on the DIII-D Tokamak," Bull. Am. Phys. Soc. 41, 1428 (1996) Abstract.

Lohr, J., D. Ponce, L. Popov, J.F. Tooker, D. Zhang, "Initial Tests and Operation of a $110 \mathrm{GHz}, 1 \mathrm{MW}$ Gyrotron With Evacuated Waveguide System on the DIII-D Tokamak," in Proc. 3rd Int. Wkshp. on Strong Microwaves in Plasmas, August 7-14, 1996, St. Petersburg, Russia, to be published; and General Atomics Report GA-A22420 (1996). 
Luce, T.C., C.C. Petty, "Dimensionless Parameter Scaling for Transport Physics and Performance Extrapolation," Bull. Am. Phys. Soc. 41, 1572 (1996) Abstract.

Luce, T.C., C.C. Petty, "Experimental Determination of the Dimensionless Scaling Parameter of Energy Transport in Tokamaks," in Proc. 22nd European Conference on Controlled Fusion and Plasma Physics, July 3-7, 1995, Bournemouth, United Kingdom, to be published (European Physical Society, Petit-Lancy, Switzerland, 1995); and General Atomics Report GA-A22096 (1995).

Luce, T.C., C.C. Petty, B. Balet, J.G. Cordey, "Implications from Dimensionless Parameter Scaling Experiments," in Proc. 16th Int. Conf. on Plasma Phys. and Controlled Nucl. Fusion Research, October 7-11, 1996, Montreal, Canada (International Atomic Energy Agency, Vienna, to be published); and General Atomics Report GA-A22467 (1996).

Luckhardt, S., J.W. Cuthbertson, J. Zhang, R.E. Stockdale, T.E. Evans, A.G. Kellman, P.L. Taylor, D.G. Whyte, "Temperature and Density Evolution Measurements and Runaway Production During Disruptions in DIII-D," Bull. Am. Phys. Soc. 41, 1433 (1996) Abstract.

Luxon, J.L., "DIII-D Team, An Overview of the DIII-D Program," in Proc. 19th Symp. on Fusion Technology, September 16-20, 1996, Lisbon, Portugal, to be published; and General Atomics Report GA-A22458 (1996).

Mahdavi, M.A., S.L. Allen, N.H. Brooks, R.J. Bastasz, L.R. Baylor, J.N. Brooks, D.A. Buchenauer, J.W. Cuthbertson, T.E. Evans, M.E. Fenstermacher, D.N. Hill, D.L. Hillis, J.T. Hogan, A.W. Hyatt, R.C. Isler, G.L. Jackson, T.C. Jernigan, R.A. Jong, C.C. Klepper, R.J. La Haye, C.J. Lasnier, A.W. Leonard, R. Maingi, P.K. Mioduszewski, R.A. Moyer, M. Murakami, L.W. Owen, T.W. Petrie, G.D. Porter, M.E. Rensink, T. Rognlien, M.J. Schaffer, G.M. Staebler, R.D. Stambaugh, D.M. Thomas, M.R. Wade, W.R. Wampler, J.G. Watkins, W.P. West, D.G. Whyte, C.P.C. Wong, R.D. Wood, DIII-D Physics and Operations Teams, "Divertor Plasma Physics Experiments on the DIII-D Tokamak," in Proc. 16th Int. Conf. on Plasma Phys. and Controlled Nucl. Fusion Research, October 7-11, 1996, Montreal, Canada (International Atomic Energy Agency, Vienna, to be published); and General Atomics Report GA-A22478 (1996).

Mahdavi, M.A., R.J. La Haye, A.W. Leonard, T.W. Petrie, R.D. Stambaugh, W.P. West, R. Maingi, L.R. Baylor, T.C. Jernigan, M.R. Wade, M. Murakami, J.G. Watkins, J. Cuthbertson, "Attainment of High Density in DIII-D H-mode Plasmas," Bull. Am. Phys. Soc. 41, 1532 (1996) Abstract.

Mahdavi, M.A., G.M. Staebler, R.D. Wood, D.G. Whyte, W.P. West, "Stability of a Radiative Mantle in ITER," in Proc. 12th Int. Conf. on Plasma Surface Interactions in Contr. Fusion Devices, May 20-24, 1996, Saint Raphael, France, to be published; and General Atomics Report GA-A22348 (1996).

Maingi, R., "Investigation of Physical Processes Limiting Plasma Density in DIII-D," Bull. Am. Phys. Soc. 41, 1501 (1996) Invited Abstract. 
Maingi, R., G.L. Jackson, M.R. Wade, M.A. Mahdavi, P.K. Mioduszewski, G. Haas, M.J. Schaffer, J.T. Hogan, "Divertor Particle Exhaust and Wall Inventory on DIII-D," in Proc. 22nd European Conference on Controlled Fusion and Plasma Physics, June 3-7, 1995, Bournemouth, United Kingdom, Vol. 19C, p. I-325.(European Physical Society, PetitLancy, Switzerland, 1995).

Maingi, R., G.L. Jackson, M.R. Wade, M.A. Mahdavi, P.K. Mioduszewski, M.J. Schaffer, J.T. Hogan, C.C. Klepper, G. Haas, "Control of Wall Particle Inventory with Divertor Pumping on DIII-D," in Nucl. Fusion 36, 245 (1996).

Maingi, R., B. Terreault, G. Haas, H.S. Bosch, S.C. Chiu, G.L. Jackson, P.K. Mioduszewski, M.A. Mahdavi, M.J. Schaffer, M.R. Wade, W. Zuzak, "Comparison of Wall/Divertor Deuterium Rention and Plasma Fueling Requirements on the DIII-D, TdeV, and ASDEX-U Tokamaks," in Proc. 12th Int. Conf. on Plasma Surface Interactions in Contr. Fusion Devices, May 20-24, 1996, Saint Raphael, France, to be published; and General Atomics Report GA-A22359 (1996).

Mauel, M.E., B.W. Rice, E.J. Strait, L.L. Lao, T.S. Taylor, K.H. Burrell, M.S. Chu, Y.B. Kim, R.J. La Haye, E.A. Lazarus, Y.R. Lin-Liu, "Production and Stability of High-Beta DIII-D Discharges with Reversed Magnetic Shear," in Proc. 22nd European Conference on Controlled Fusion and Plasma Physics, July 3-7, 1995, Bournemouth, United Kingdom, to be published (European Physical Society, Petit-Lancy, Switzerland, 1995); and General Atomics Report GA-A22101 (1995).

McKee, Jr., G.R., D.L. Hillis, R.C. Isler, C.C. Klepper, M.R. Wade, N.H. Brooks, W.P. West, "Neutral and Low-Charge-State Helium and Neon Spectroscopy Measurements During Radiative Divertor Experiments on DIII-D," Bull. Am. Phys. Soc. 41, 1431 (1996) Abstract.

Miller, R.L., Y.R. Lin-Liu, A.D. Turnbull, V.S. Chan, L.D. Pearlstein, O. Sauter, "Stable Equilibria for Bootstrap-Current Driven Low Aspect Ratio Tokamaks," Bull. Am. Phys. Soc. 41, 1404 (1996) Abstract.

Miller, R.L., Y.-R. Lin-Liu, A.D. Turnbull, V.S. Chan, L.D. Pearlstein, O. Sauter, L. Villard, "Stable Bootstrap-Current Driven Equilibria for Low Aspect Ratio Tokamaks," in Proc. ISPP/ Wkshp. on Theory of Fusion Plasmas, August 26-31, 1996, Varenna, Italy, to be published; and General Atomics Report GA-A22433 (1996).

Miller, R.L., Y.R. Lin-Liu, A.D. Turnbull, V.S. Chan, L.D. Pearlstein, O. Sauter, L. Villard, "Stable Equilibria for Bootstrap-Current Driven Low Aspect Ratio Tokamaks," Phys. Plasmas 4, 1062 (1997).

Monier-Garbet, P., K.H. Burrell, F.L. Hinton, J. Kim, X. Garbet, R.J. Groebner, "Effects of Neutrals on Plasma Rotation in DIII-D," Nucl. Fusion 36, 1217 (1996).

Moyer, R.A., J. Cuthbertson, E.J. Doyle, C.L. Rettig, T.L. Rhodes, R.J. Groebner, K.H. Burrell, J.G. Watkins, R. Maingi, G.D. Porter, "Turbulence Suppression and Transport Barrier Formation in Very Slow L-H Transitions," Bull. Am. Phys. Soc. 41, 1574 (1996) Abstract. 
Murakami, M., E.F. Jaeger, F.W. Baity, C.C. Petty, C.B. Forest, S.C. Chiu, J.S. deGrassie, J.M. Lohr, R.I. Pinsker, R. Prater, "Fast Wave Electron Heating and Current Drive and Comparison with RF Modeling in DIII-D,” Bull. Am. Phys. Soc. 41, 1388 (1996) Abstract.

Navratil, G.A., E.A. Lazarus, C.M. Greenfield, B.W. Rice, "High Fusion Performance NCS Plasmas in DIII-D," Bull. Am. Phys. Soc. 41, 1386 (1996) Abstract.

Nilson, D.G., N.H. Brooks, J.P. Smith, R.T Snider, "Diagnostics for the DIII-D Radiative Divertor," in Proc. 16th IEEE/NPS Symp. on Fusion Engineering, September 30October 5, 1995, Champaign, Illinois, Vol. 1, p. 806 (Institute of Electrical and Electronics Engineers, Inc., Piscataway, New Jersey, 1996).

O’Neill, R.C., R.W. Callis, W.P. Cary, J.L. Doane, R. Gallix, T.R. Hodapp, J.M. Lohr, T.C. Luce, and C.P. Moeller, "ECH Mirror Interface Tank for $110 \mathrm{GHz}, 1 \mathrm{MW}$ Gyrotron," in Proc. 16th IEEE/NPS Symp. on Fusion Engineering, September 30-October 5, 1995, Champaign, Illinois, Vol. 1, p. 844 (Institute of Electrical and Electronics Engineers, Inc., Piscataway, New Jersey, 1996).

Olstad, R.A., J.L. Doane, C.P. Moeller, R.C. O'Neill, M. Di Martino, "High-Power Corrugated Waveguide Components for Millimeter Wave Fusion Heating Systems," in Proc. 19th Symp. on Fusion Technology, September 16-20, 1996, Lisbon, Portugal, to be published; and General Atomics Report GA-A22466 (1996).

Osborne, T.H., A.W. Leonard, D.G. Porter, The DIII-D Team, "ELM Studies on DIII-D," Bull. Am. Phys. Soc. 41, 1534 (1996) Abstract.

Owen, L., J. Hogan, M.R. Wade, R.C. Isler, R. Maingi, D. Coster, "Divertor Enrichment Modeling for DIII-D," Bull. Am. Phys. Soc. 41, 1431 (1996) Abstract.

Parks, P.B., D. Whyte, R. Bastasz, N.H. Brooks, W.R. Wampler, W.P. West, and C.P.C. Wong, "First Measurements of the Ion Energy Distribution at the Divertor Strike Point During DIII-D Disruptions," in Proc. 16th IEEE/NPS Symp. on Fusion Engineering, September 30-October 5, 1995, Champaign, Illinois, Vol. 1, p. 821 (Institute of Electrical and Electronics Engineers, Inc., Piscataway, New Jersey, 1996).

Peebles, W.A., C.L. Rettig, J. Lohr, K.H. Burrell, "Electron Temperature Fluctuation Measurements in DIII-D," Bull. Am. Phys. Soc. 41, 1572 (1996) Abstract.

Penaflor, B., J.R. Ferron, M.L. Walker, "A Structured Architecture for Advanced Plasma Control Experiments," in Proc. 19th Symp. on Fusion Technology, September 16-20, 1996, Lisbon, Portugal, to be published; and General Atomics Report GA-A22545 (1996).

Petrie, T.W., S.L. Allen, T.N. Carlstrom, D.N. Hill, R. Maingi, D.G. Nilson, M.D. Brown, D.A. Buchenauer, T.E. Evans, M.E. Fenstermacher, R.A. Jong, C.J. Lasnier, A.W. Leonard, M.A. Mahdavi, G.D. Porter, M.R. Wade, W.P. West, "Investigation of Electron Parallel Pressure Balance in the Scrape-Off Layer of Deuterium-Based Radiative Divertor Discharges in DIII-D," in Proc. 12th Int. Conf. on Plasma Surface Interactions in Contr. Fusion Devices, May 20-24, 1996, Saint Raphael, France, to be published; and General Atomics Report GA-A22349 (1996). 
Petrie, T.W., D.N. Hill, S.L. Allen, N.H. Brooks, D. Buchenauer, J.W. Cutherbertson, T.E. Evans, P.H. Ghendrih, C. Lasner, A.W. Leonard, R. Maingi, "Radiative Divertor Experiments in DIII-D with $\mathrm{D}_{2}$ Injection," Nucl. Fusion 37, No. 3, 321 (1997).

Petrie, T.W., A.W. Leonard, M.A. Mahdavi, W.P. West, S.L. Allen, M.E. Fenstermacher, D.N. Hill, C.J. Lasnier, R.D. Wood, R. Maingi, D.G. Whyte, "Divertor and X-Point MARFE Behavior in DIII-D H-mode Discharges," Bull. Am. Phys. Soc. 41, 1430 (1996) Abstract.

Petty, C.C., C.B. Forest, F.W. Baity, S.C. Chiu, J.S. deGrassie, R.J. Groebner, Y.R. Lin-Liu, T.C. Luce, R.I. Pinsker, M. Porkolab, R. Prater, "Current Profile Evolution During Fast Wave Current Drive on the DIII-D Tokamak," in Proc. 11th Top. Con. on Radio Frequency Power in Plasmas, May 17-19, 1995, Palm Springs, California, p. 193 (American Institute of Physics, New York, 1996).

Petty, C.C., T.C. Luce, "Nondimensional Transport Experiments in DIII-D," Bull. Am. Phys. Soc. 41, 1388 (1996) Abstract.

Petty, C.C., T.C. Luce, B. Balet, J.P. Christiansen, J.G. Cordey, "Nondimensional Transport Experiments on DII-D and Projections to an Ignition Tokamak," in Proc. 23rd European Conf. on Contr. Fusion and Plasma Phys., June 24-28, 1996, Kiev, Ukraine, Vol. 20C, Part I, p. 211 (European Physical Society, Petit-Lancy, Switzerland, 1996).

Phelps, D.A., "Broadband Tunable and ELM Resilient Traveling Wave Antennas for Plasma Heating and Shaping in DIII-D," Bull. Am. Phys. Soc. 41, 1429 (1996) Abstract.

Phelps, D.A., H. Ikezi and C.P. Moeller, "A High Power Experimental Traveling Wave Antenna for Fast Wave Heating and Current Drive in DIII-D and Relevance to ITER," in Proc. 16th IEEE/NPS Symp. on Fusion Engineering, September 30-October 5, 1995, Champaign, Illinois, Vol. 2, p. 848 (Institute of Electrical and Electronics Engineers, Inc., Piscataway, New Jersey, 1996).

Phelps, D.A., C.P. Moeller, H. Ikezi, S.C. Chiu, "First Demonstration of a Traveling Wave Antenna in a Tokamak and Relevance to the JFT-2M Combline," in Proc. 11th Top. Con. on Radio Frequency Power in Plasmas, May 17-19, 1995, Palm Springs, California, to be published; and General Atomics Report GA-A22051 (1995).

Phillips, J.C., D.H. Kellman, R. Hong, J. Kim, G.M. Laughon, "Five Second Helium Neutral Beam Injection Using Argon-Frost Cryopumping Techniques," in Proc. 16th IEEE/NPS Symp. on Fusion Engineering, September 30-October 5, 1995, Champaign, Illinois, Vol. 2, p. 852 (Institute of Electrical and Electronics Engineers, Inc., Piscataway, New Jersey, 1996).

Pinsker, R.I., F.W. Baity, S.C. Chiu, J.S. deGrassie, C.B. Forest, H. Ikezi, M. Murakami, C.C. Petty, R. Prater, D.W. Swain, DIII-D Group, "Direct Electron Heating with Directional Fast Wave Launch on DIII-D," in Proc. 11th Top. Con. on Radio Frequency Power in Plasmas, May 17-19, 1995, Palm Springs, California, to be published; and General Atomics Report GA-A22081 (1995). 
Pinsker, R.I., C.P. Moeller, C.C. Petty, D.A. Phelps, T. Ogawa, Y. Miura, H. Ikezi, J.S. deGrassie, R.W. Callis, R.H. Goulding, F.W. Baity, "Development of Fast Wave Systems Tolerant of Time-Varying Loading," in Proc. 19th Symp. on Fusion Technology, September 16-20, 1996, Lisbon, Portugal, to be published; and General Atomics Report GA-A22450 (1996).

Pinsker, R.I., C.C. Petty, D.A. Phelps, C.P. Moeller, R.W. Callis, T. Ogawa, Y. Miura, "First High Power Results from the General Atomics Combline on the JFT-2M Tokamak," Bull. Am. Phys. Soc. 41, 1429 (1996) Abstract.

Popov, A.M., Y.Q. Liu, A.D. Turnbull, M.S. Chu, "Nonlinear MHD Simulation of Negative Central Shear Discharges in the DIII-D Tokamak," Bull. Am. Phys. Soc. 41, 1569 (1996) Abstract.

Popova, N.N., Y.Q. Liu, A.M. Popov, A.D. Turnbull, M.S. Chu, "Nonlinear Simulations of Magnetic Field Lines Stochastization in the Negative Central Shear Experiments in the DIII-D Tokamak," Bull. Am. Phys. Soc. 41, 1570 (1996) Abstract.

Prater, R., M.E. Austin, F.W. Baity, S.C. Chiu, R.W. Callis, W.P. Cary, J.S. deGrassie, R.L. Freeman, C.B. Forest, S.W. Ferguson, H. Ikezi, E.F. Jaeger, E. Joffrin, J.H. Lee, Y.-R. Lin-Liu, T.C. Luce, M. Murakami, C.C. Petty, R.I. Pinsker, D.A. Phelps, M. Porkolab, D.W. Swain, The DIII-D Team, "Fast Wave Heating and Current Drive in DIII-D in Discharges with Negative Central Shear," in Proc. 16th Int. Conf. on Plasma Phys. and Controlled Nucl. Fusion Research, October 7-11, 1996, Montreal, Canada (International Atomic Energy Agency, Vienna, to be published); and General Atomics Report GA-A22449 (1996).

Prater, R., F.W. Baity, W.P. Cary, S.C. Chiu, J.S. deGrassie, C.B. Forest, R.W. Harvey, H. Ikezi, E.F. Jaeger, K. Kupfer, Y.R. Lin-Liu, "First Results on Fast Wave Current Drive in Advanced Tokamak Discharges in DIII-D," in Proc. 22nd European Conference on Controlled Fusion and Plasma Physics, July 3-7, 1995, Bournemouth, United Kingdom, Vol. 1, p. 381 (European Physical Society, Petit-Lancy, Switzerland, 1995).

Prater, R., R.W. Callis, J.M. Lohr, T.C. Luce, J.F. Tooker, D. Zhang, L. Popov, M. Murakami, J.A. Konings, "Initial Electron Cyclotron Heating Experiments at $110 \mathrm{GHz}$ in DIII-D," Bull. Am. Phys. Soc. 41, 1388 (1996) Abstract.

Reis, E.E., R.H. Ryder, "Creep-Fatigue Damage in OFHC Coolant Tubes for Plasma Facing Components," in Proc. 19th Symp. on Fusion Technology, September 16-20, 1996, Lisbon, Portugal, to be published; and General Atomics Report GA-A22436 (1996).

Reis, E.E., J.P. Smith, C.B. Baxi, A.S. Bozek, E. Chin, M.A. Hollerbach, G.J. Laughon, D.L. Sevier, "Structural Design of the DIII-D Radiative Divertor," in Proc. 19th Symp. on Fusion Technology, September 16-20, 1996, Lisbon, Portugal, to be published; and General Atomics Report GA-A22445 (1996).

Rettig, C.L., E.J. Doyle, T.L. Rhodes, W.A. Peebles, K.H. Burrell, C.M. Greenfield, R.J. Fonck, B.W. Rice, E.A. Lazarus, "Core Turbulence Evolution and Characteristics During Negative Central Shear Discharges in DIII-D," Bull. Am. Phys. Soc. 41, 1387 (1996) Abstract. 
Rewoldt, G., L.L. Lao, W.M. Tang, "Microstability Analysis of DIII-D High-Performance Discharges," in Phys. Plasmas 3, 4074 (1996).

Rhodes, T.L., E.J. Doyle, C.L. Rettig, K.W. Kim, W.A. Peebles, R.J. Fonck, C.M. Greenfield, "Reflectometer Measurements of Core Fluctuation Behavior in NCS and Other Regimes," Bull. Am. Phys. Soc. 41, 1572 (1996) Abstract.

Rice, B.W., "q-Profile Measurements with Motional Stark Effect in DIII-D," in Proc. 7th Int. Conf. on Plasma Phys. and Contr. Nucl. Fusion, November 28-December 1, 1995, Tokai, Japan, to be published; and General Atomics Report GA-A22269 (1995).

Rice, B.W., K.H. Burrell, L.L. Lao, G. Navatril, B.W. Stallard, E.J. Strait, T.S. Taylor, M.E. Austin, T.A. Casper, M.S. Chu, C.B. Forest, P. Gohil, R.J. Groebner, W.W. Heidbrink, A.W. Hyatt, H. Ikezi, R.J. La Haye, E.A. Lazarus, Y.R. Lin-Liu, M.E. Mauel, W.H. Meyer, C.L. Rettig, D.P. Schissel, H.E. St. John, P.L. Taylor, A.D. Turnbull, and the DIII-D Team, "Demonstration of High Performance Negative Central Magnetic Shear Discharges on the DIII-D Tokamak," in Phys. Plasmas 3 (5), 1983 (1996).

Rice, B.W., B.W. Stallard, E.J. Strait, T.C. Luce, T.S. Taylor, C.M. Greenfield, F. Soldner, G. Sips, E.J. Lazarus, R. Maingi, M.R. Wade, "Extension of High-Performance NCS Operating Regimes to Low-Triangularity Plasmas, with Implications for JET/TTER," Bull. Am. Phys. Soc. 41, 1386 (1996) Abstract.

Riggs, S., R. Hong, and D. Kessler, "Neutral Beam Shine-Through Power and Its Dependence On The Line Density Of The DIII-D Plasma," in Proc. 16th IEEE/NPS Symp. on Fusion Engineering, September 30-October 5, 1995, Champaign, Illinois, Vol. 2, p. 856 (Institute of Electrical and Electronics Engineers, Inc., Piscataway, New Jersey, 1996).

Robinson, J.I., and J.T. Scoville, "Development of a New Error Field Correction Coil (C-coil) for DIII-D," in Proc. 16th IEEE/NPS Symp. on Fusion Engineering, September 30October 5, 1995, Champaign, Illinois, Vol. 1, p. 829 (Institute of Electrical and Electronics Engineers, Inc., Piscataway, New Jersey, 1996).

Rothweil, D.A., D.A. Phelps, and R. Doerner, "Interpretation of PISCES-A RF Antenna System Experimental Results," in Proc. 16th IEEE/NPS Symp. on Fusion Engineering, September 30-October 5, 1995, Champaign, Illinois, Vol. 2, p. 968 (Institute of Electrical and Electronics Engineers, Inc., Piscataway, New Jersey, 1996).

Ruskov, E., W.W. Heidbrink, M.A. vanZeeland, "Analysis of Beam-Driven 'BAE' Modes," Bull. Am. Phys. Soc. 41, 1571 (1996) Abstract.

Schaffer, M.J., T.E. Evans, D.A. Humphreys, A.W. Hyatt, A.G. Kellman, R.J. La Haye, "VDE Disruption Halo Currents: Rotation and Peaking," Bull. Am. Phys. Soc. 41, 1432 (1996) Abstract.

Schaffer, M.J., R. Maingi, A.W. Hyatt, M.A. Mahdavi, "Increased Divertor Exhaust by Electrical Bias in DIII-D," in Nucl. Fusion Lett. 36, 495 (1996). 
Schaffer, M.J., M.R. Wade, R. Maingi, P. Monier-Garbet, W.P. West, D.G. Whyte, R.D. Wood, M.A. Mahdavi, "Direct Measurement of Divertor Neon Enrichment in DIII-D," in Proc. 12th Int. Conf. on Plasma Surface Interactions in Contr. Fusion Devices, May 20-24, 1996, Saint Raphael, France, to be published; and General Atomics Report GA-A22343 (1996).

Schissel, D.P., DIII-D Education Outreach Team, "Advancing Precollege Science and Mathematics Education in San Diego County," Bull. Am. Phys. Soc. 41, 1456 (1996) Abstract.

Schissel, D.P., J.C. DeBoo, C.M. Greenfield, K.H. Burrell, L.L. Lao, E.J. Strait, E.A. Lazarus, M. Murakami, B.W. Rice, B.W. Stallard, G.A. Navratil, "Energy Transport in Neutral Beam Heated DIII-D Discharges with Negative Magnetic Shear," Bull. Am. Phys. Soc. 41, 1571 (1996) Abstract.

Schissel, D.P., C.M. Greenfield, J.C. DeBoo, L.L. Lao, E.A. Lazarus, G.A. Navratil, B.W. Rice, G.M. Staebler, B.W. Stallard, E.J. Strait, H.E. St. John, M.E. Austin, K.H. Burrell, T.A. Casper, D.R. Baker, V.S. Chan, E.J. Doyle, J.R. Ferron, C.B. Forest, P. Gohil, R.J. Groebner, W.W. Heidbrink, R.-M. Hong, A.W. Howald, C.-L. Hsieh, A.W. Hyatt, G.L. Jackson, J. Kim, C.J. Lasnier, A.W. Leonard, J. Lohr, R.J. La Haye, R. Maingi, R.L. Miller, M. Murakami, T.H. Osborne, C.C. Petty, C.L. Rettig, T.L. Rhodes, S.A. Sabbagh, J.T. Scoville, R.T. Snider, R.D. Stambaugh, R.E. Stockdale, P.L. Taylor, T.S. Taylor, D.M. Thomas, M.R. Wade, R.E. Waltz, R.D. Wood, D.G. Whyte, "Local Analysis of Confinement and Transport in Neutral Beam Heated DIII-D Discharges with Negative Magnetic Shear," in Proc. 16th Int. Conf. on Plasma Phys. and Controlled Nucl. Fusion Research, October 7-11, 1996, Montreal, Canada (International Atomic Energy Agency, Vienna, to be published); and General Atomics Report GA-A22479 (1996).

Scoville, J.T., R.J. La Haye, "Multi-mode Error Field Correction,” Bull. Am. Phys. Soc. 41, 1570 (1996) Abstract.

Sevier, D.L., C.B. Baxi, D.N. Hill, E.E. Reis, G.W. Silke, C.P.C. Wong, "Implications of Steady-State Operation on Divertor Design," in Proc. 12th Top. Mtg. on Technology Fusion Energy, June 16-20, 1996, Reno, Nevada, to be published; General Atomics Report GA-A22388 (1996).

Simonen, T.C. for The DIII-D Team, "DIII-D Research Program Status and Plans," Bull. Am. Phys. Soc. 41, 1532 (1996) Abstract.

Smith, J.P., C.B. Baxi, A.S. Bozek, E. Chin, M.A. Hollerbach, W.R. Johnson, G.J. Laughon, R.D. Phelps, K.M. Redler, E.E. Reis, and D.L. Sevier, "Engineering Design of a Radiative Divertor for DIII-D," in Proc. 16th IEEE/NPS Symp. on Fusion Engineering, September 30-October 5, 1995, Champaign, Illinois, Vol. 1, p. 801 (Institute of Electrical and Electronics Engineers, Inc., Piscataway, New Jersey, 1996).

Smith, J.P., C.B. Baxi, A.S. Bozek, E. Chin, M.A. Hollerbach, W.R. Johnson, G.J. Laughon, E.E. Reis, D.L. Sevier, D.G. Nilson, "The DIII-D Radiative Divertor Project, Status and Plans," in Proc. 12th Top. Mtg. on Technology Fusion Energy, June 16-20, 1996, Reno, Nevada, to be published; and General Atomics Report GA-A22389 (1996). 
Smith, J.P., W.R. Johnson, C.B. Baxi, "Manufacturing Development of Low Activation Vanadium Alloys," in Proc. 12th Top. Mtg. on Technology Fusion Energy, June 16-20, 1996, Reno, Nevada, to be published; and General Atomics Report GA-A22382 (1996).

Smith, J.P., W.R. Johnson, E.E. Reis, "Fabrication Development and Usage of Vanadium Alloys in DIII-D," in Proc. 19th Symp. on Fusion Technology, September 16-20, 1996, Lisbon, Portugal, to be published; and General Atomics Report GA-A22452 (1996).

Smith, J.P., W.R. Johnson, R.D. Stambaugh, P.W. Trester, D. Smith, and E. Bloom "Vanadium Alloys For The Radiative Divertor Program of DIII-D," in Proc. 16th IEEE/NPS Symp. on Fusion Engineering, September 30-October 5, 1995, Champaign, Illinois, Vol. 1, p. 858 (Institute of Electrical and Electronics Engineers, Inc., Piscataway, New Jersey, 1996).

Snider, R.T., T.N. Carlstrom, T.R. Hodapp, F.C. Jobes, W.A. Peebles, “Application of Interferometry and Faraday Rotation Techniques for Density Measurements on the Next Generation of Tokamaks," presented at 11th Top. Conf. on High Temperature Plasma Diagnostics, May 12-16, 1996, Monterey, California, to be published in Rev. Sci. Instrum.; and General Atomics Report GA-A22328 (1996).

St. John, H.E., J.C. DeBoo, C.M. Greenfield, D.P. Schissel, B.W. Stallard, "Application of Empirically Derived MHD Metrics in Current Drive and Transport Analysis of NCS Discharges in DII-D," Bull. Am. Phys. Soc. 41, 1571 (1996) Abstract.

Staebler, G.M., "The Critical Point for the Onset of Divertor Energy Flux Asymmetry in Tokamaks," to be published in Nucl. Fusion; and General Atomics Report GA-A22053 (1995).

Staebler, G.M., R.E. Waltz, M. Kotschenreuther, "Gyrokinetc Stability of DIII-D NCS Discharges,” Bull. Am. Phys. Soc. 41, 1387 (1996) Abstract.

Stallard, B.W., T.A. Casper, B.W. Rice, C.M. Greenfield, D.P. Schissel, H.E. St. John, K.H. Burrell, J.C. DeBoo, P. Gohil, R.J. Groebner, L.L. Lao, E.A. Lazarus, C.L. Rettig, "Reduced Transport in Negative and Weak Shear Discharges in DIII-D," Bull. Am. Phys. Soc. 41, 1387 (1996) Abstract.

Stambaugh, R.D., V.S. Chan, P.M. Anderson, C.B. Baxi, R.W. Callis, H.K. Chiu, C.B. Forest, R.-M. Hong, T.H. Jensen, L.L. Lao, J.A. Leuer, M.A. Mahdavi, R.L. Miller, A. Nerem, R. Prater, P.A. Politzer, M.J. Schaffer, D.L. Sevier, T.S. Taylor, A.D. Turnbull, C.P.C. Wong, "The Spherical Torus Approach to Magnetic Fusion Development," in Proc. 12th Top. Mtg. on Technology Fusion Energy, June 16-20, 1996, Reno, Nevada, to be published; and General Atomics Report GA-A22393 (1996).

Stephens, R.B., G. Collins, “Analysis of Integrating Sphere Performance for IR Enhanced DT Layering," presented at 11th Target Fabrication Specialists Meeting, September 8-12, 1996, Orcas Island, Washington, to be published in Fusion Technology; and General Atomics Report GA-A22477 (1996).

Strait, E.J., "Frequency Response of Metal-Clad Inductive Magnetic Field Probes," in Rev. Sci. Instrum. 67, 2538 (1996). 
Strait, E.J., "Stability of Negative Central Magnetic Shear Discharges in the DIII-D Tokamak," Bull. Am. Phys. Soc. 41, 1442 (1996) Invited Abstract.

Strait, E.J., J.D. Broesch, R.T. Snider, M.L. Walker, “A Hybrid Digital-Analog Long Pulse Integrator," presented at 11th Top. Conf. on High Temperature Plasma Diagnostics, May 12-16, 1996, Monterey, California, to be published in Rev. Sci. Instrum; and General Atomics Report GA-A22338 (1996).

Strait, E.J., T.A. Casper, M.S. Chu, J.R. Ferron, C.B. Forest, C.M. Greenfield, A.W. Hyatt, L.L. Lao, R.J. La Haye, E.A. Lazarus, G.A. Navratil, B.W. Rice, A.C.C. Sips, F.X. Soldner, B.W. Stallard, T.S. Faylor, A.D. Turnbull, "Improved Fusion Performance in Low-q, Low Triangularity Plasmas with Negative Central Magnetic Shear," in Proc. 23rd European Conf. on Contr. Fusion and Plasma Phys., June 24-28, 1996, Kiev, Ukraine, Vol. 20C, Part III, p. 1493 (European Physical Society, Petit-Lancy, Switzerland, 1996).

Strait, E.J., L.L. Lao, M.E. Mauel, B.W. Rice, T.S. Taylor, K.H. Burrell, M.S. Chu, E.A. Lazarus, T.H. Osborne, S.J. Thompson, A.D. Turnbull, "Enhanced Confinement and Stability in DIII-D Discharges with Reversed Magnetic Shear," in Phys. Rev. Lett. 75, 4421 (1995).

Taylor, P.L., A.G. Kellman, T.E. Evans, D.A. Humphreys, A.W. Hyatt, R.L. Lee, M.J. Schaffer, J.W. Cuthbertson, S. Luckhardt, J. Zhang, T. Jernigan, D.G. Whyte, "Disruption Mitigation Using Impurity Pellet Injection in DIII-D,” Bull. Am. Phys. Soc. 41, 1432 (1996) Abstract.

Taylor, P.L., A.G. Kellman, B.W. Rice, R. Bastasz, N.H. Brooks, T.E. Evans, K.L. Holtrop, D.A. Humphreys, A.W. Hyatt, R.L. Lee, D.G. Whyte, "DIII-D Disruption Experiment Results," in Proc. 22nd European Conference on Controlled Fusion and Plasma Physics, July 3-7, 1995, Bournmouth, United Kingdom, to be published (European Physical Society, Petit-Lancy, Switzerland, 1995); and General Atomics Report GA-A22082 (1995).

Taylor, P.L., A.G. Kellman, B.W. Rice, D.A. Humphreys, "Experimental Measurements of the Current, Temperature, and Density Profile Changes during a Disruption in the DIII-D Tokamak," in Phys. Rev. Lett. 76 (6), 916 (1996).

Taylor, T.S., J.R. Ferron, L.L. Lao, E.J. Strait, B.W. Rice, "Evidence for High Edge Current Density and Its Impact on Plasma Control," Bull. Am. Phys. Soc. 41, 1569 (1996) Abstract.

Thomas, D.M., "Visualization of Density Structures in Tokamak Edge Plasmas Using Lithium Beam Fluorescence," in Proc. IEEE Transactions on Plasma Science 24 (1), 27 (1996).

Thomas, D.M., K.H. Burrell, R.J. Groebner, P. Gohil, D.H. Kaplan, C.C. Makariou, R.P. Seraydarian, "A Fast CCD Detector for Charge Exchange Recombination Spectroscopy on the DIII-D Tokamak," presented at 11th Top. Conf. on High Temperature Plasma Diagnostics, May 12-16, 1996, Monterey, California, to be published in Rev. Sci. Instrum; and General Atomics Report GA-A22329 (1996).

Thomas, D.M., T.N. Carlstrom, R.J. Groebner, A.W. Leonard, T.L. Rhodes, C.L. Rettig, "Parameters Affecting the H-L Transition on DIII-D," Bull. Am. Phys. Soc. 41, 1573 (1996) Abstract. 
Turnbull, A.D., E.J. Strait, L.L. Lao, M.S. Chu, R.L. Miller, T.S. Taylor, C.M. Greenfield, R.J. La Haye, R.T. Snider, B.W. Rice, E.A. Lazarus, G.A. Navratil, "Beta Limiting Instabilities in Negative Central Shear Discharges," Bull. Am. Phys. Soc. 41, 1569 (1996) Abstract.

VanderLaan, J.F., S. Miller, B.B. McHarg, Jr., P.A. Henline, "Handling and Archiving of Magnetic Fusion Data at DIII-D," in Proc. 16th IEEE/NPS Symp. on Fusion Engineering, September 30-October 5, 1995, Champaign, Illinois, Vol. 2, p. 881 (Institute of Electrical and Electronics Engineers, Inc., Piscataway, New Jersey, 1996).

Wade, M.R., S.L. Allen, R.D. Wood, M.J. Schaffer, W.P. West, R. Maingi, D.G. Whyte, "Neon Enrichment and Particle Balance During 'Puff and Pump' Experiments on DIII-D," Bull. Am. Phys. Soc. 41, 1533 (1996) Abstract.

Wade, M.R., D.L. Hillis, K.H. Burrell, T.C. Luce, R. Maingi, P.K. Mioduszewski, C.C. Petty, W.P. West, R.-M. Hong, D.H. Kellman, J.C. Phillips, DIII-D Team, "Experimental Evidence for the Suitability of ELMing H-Mode Operation in ITER with Regard to Core Transport of Helium," in Proc. 16th Int. Conf. on Plasma Phys. and Controlled Nucl. Fusion Research, October 7-11, 1996, Montreal, Canada (International Atomic Energy Agency, Vienna, to be published); and General Atomics Report GA-A22473 (1996).

Wade, M.R., D.G. Whyte, R.D. Wood, W.P. West, "Charazerization of Core Impurity Transport and Accumulation in Various Operating Regimes in DIII-D," in Proc. 23rd European Conf. on Contr. Fusion and Plasma Phys., June 24-28, 1996, Kiev, Ukraine, Vol. 20C, Part I, p. 283 (European Physical Society, Petit-Lancy, Switzerland, 1996).

Walker, M., J.R. Ferron, B. Penaflor, D.A. Humphreys, J.A. Leuer, A.W. Hyatt, C.B. Forest, J.T. Scoville, B.W. Rice, E.A. Lazarus, T.W. Petrie, S.L. Allen, G.L. Jackson, and R. Maingi, "Status of DIII-D Plasma Control," in Proc. 16th IEEE/NPS Symp. on Fusion Engineering, September 30-October 5, 1995, Champaign, Illinois, Vol. 2, p. 885 (Institute of Electrical and Electronics Engineers, Inc., Piscataway, New Jersey, 1996).

Wang, Z., J. Lohr, G.L Bell, C. Hsieh, J. Luo, R.E. Stockdale, J.B. Wilgen, J. Zhang, "Study of Electron Temperature Profile Evolution During L-H Transition with Measurement of Electron Cyclotron Emission on DIII-D," in Proc. 9th Joint Workshop on Electron Cyclotron Emission and Electron Cyclotron Resonance Heating, January 22-26, 1995, Borrego Springs, California, to be published (World Scientific Publishing Co., Singapore, Japan 1995); and General Atomics Report GA-A22038 (1995).

Watkins, J.G., D.A. Buchenauer, D.N. Hill, D.G. Porter, T.N. Carlstrom, J.W. Cuthbertson, R.A. Moyer, "Scaling of Density and Temperature Gradients in the DIII-D Divertor," Bull. Am. Phys. Soc. 41, 1431 (1996) Abstract.

Watkins, J.G., R.A. Moyer, J.W. Cuthbertson, D.A. Buchenauer, T.N. Carlstrom, D.N. Hill, M. Ulrickson, "Reciprocating and Fixed Probe Measurements of $n_{e}$ and $T_{e}$ in the DIII-D Divertor," in Proc. 12th Int. Conf. on Plasma Surface Interactions in Contr. Fusion Devices, May 20-24, 1996, Saint Raphael, France, to be published; and General Atomics Report GA-A22362 (1996). 
Weschenfelder, F., G.L. Jackson, N.H. Brooks, R.L. Lee, W.P. West, P. Wienhold, J. Winter, "Three-Filter Colorimetry in DIII-D to Measure In-Situ Erosion and Deposition of Plasma Facing Surfaces," Rev. Sci. Instrum. 66, 5257 (1995).

West, W.P., S.L. Allen, N.H. Brooks, D.A. Buchenauer, T.N. Carlstrom, J.W. Cuthbertson, E.J. Doyle, T.E. Evans, M.E. Fenstermacher, D.N. Hill, A.W. Hyatt, R.C. Isler, G.L. Jackson, R.A. Jong, C.C. Klepper, C.J. Lasnier, A.W. Leonard, M.A. Mahdavi, R. Maingi, G.R. McKee, W.H. Meyer, R.A. Moyer, D.G. Nilson, T.W. Petrie, G.D. Porter, T.L. Rhodes, M.J. Schaffer, R.D. Stambaugh, D.M. Thomas, S.N. Tugarinov, M.R. Wade, J.G. Watkins, D.G. Whyte, R.D. Wood, "Divertor Plasma Studies on DIII-D - Experiment and Modeling," in Proc. 8th Int. Conf. on Plasma Phys., September 9-11, 1996, Nagoya, Japan, to be published; and General Atomics Report GA-A22461 (1996).

West, W.P., N.H. Brooks, S.L. Allen, G.D. Porter, R.D. Wood, R.C. Isler, M.R. Wade, G.R. McKee, D.G. Whyte, "Modeling of Impurity Spectroscopy in the DIII-D Divertor," Bull. Am. Phys. Soc. 41, 1431 (1996) Abstract.

West, W.P., T.E. Evans, N.H. Brooks, M.E. Fenstermacher, S. Hirshman, R.C. Isler, A.W. Leonard, G.D. Porter, M.R. Wade, D.G. Whyte, R.D. Wood, "Modeling of Impurity Spectroscopy in the Divertor and SOL of DIII-D Using the 1D Multifluid Model NEWT1D," in Proc. 23rd European Conf. on Contr. Fusion and Plasma Phys., June 24-28, 1996, Kiev, Ukraine Vol. 20C, Part II, p. 767 (European Physical Society, Petit-Lancy, Switzerland, 1996).

Whyte, D.G., J.N. Brooks, C.P.C. Wong, W.P. West, R.J. Bastasz, W.R. Wampler, J. Rubinstein, "DiMES Divertor Erosion Experiments on DIII-D," in Proc. 12th Int. Conf. on Plasma Surface Interactions in Contr. Fusion Devices, May 20-24, 1996, Saint Raphael, France, to be published; and General Atomics Report GA-A22342 to be published.

Whyte, D.G., T.E. Evans, A.G. Kellman, D.A. Humphreys, P.L. Taylor, R.T. Snider, W.P. West, J.W. Cuthbertson, R.D. Wood, "Spectroscopic Investigation of Disruptions on DIII-D," Bull. Am. Phys. Soc. 41, 1432 (1996) Abstract.

Winter, Partricia S., "General Atomics Science Education Outreach Programs," Bull. Am. Phys. Soc. 41, 1456 (1996) Abstract.

Wong, C.P.C., R.J. Bastasz, D.G. Whyte, W.R. Wampler, W.P. West, "Results from the Divertor Materials Evaluation Studies (DiMES) at DIII-D," in Proc. 12th Top. Mtg. on Technology Fusion Energy, June 16-20, 1996, Reno, Nevada, to be published; and General Atomics Report GA-A22392 (1996).

Wood, R.D., S.L. Allen, M.E. Fenstermacher, C.J. Lasnier, R.C. Isler, M.R. Wade, A.W. Leonard, M.J. Schaffer, W.P. West, "Measurements of Divertor Impurity Emissions on DIII-D," Bull. Am. Phys. Soc. 41, 1533 (1996) Abstract.

Wood, R.D., R.C. Isler, S.L. Allen, M.E. Fenstermacher, C.J. Lasnier, A.W. Leonard, W.P. West, "Measurements of Divertor Impurity Concentrations on DIII-D," in Proc. 23rd European Conf. on Contr. Fusion and Plasma Phys., June 24-28, 1996, Kiev, Ukraine, Vol. 20C, Part II, p. 763 (European Physical Society, Petit-Lancy, Switzerland, 1996). 
Wright, A.L., J. Tooker, J.C. Allen, W.P. Cary, and T.E. Harris, "ECH Control System for New $1 \mathrm{MW} 110 \mathrm{GHz}$ Gyrotrons at DIII-D," in Proc. 16th IEEE/NPS Symp. on Fusion Engineering, September 30-October 5, 1995, Champaign, Illinois, Vol. 2, p. 889 (Institute of Electrical and Electronics Engineers, Inc., Piscataway, New Jersey, 1996).

Wroblewski, D., G.L. Jahns, J.A. Leuer, J.R. Ferron, A.G. Kellman, “Application of Neural Networks for Real Time Determination of High- $\beta$ Disruption Boundary and Current Profile Parameters," Bull. Am. Phys. Soc. 41, 1433 (1996) Abstract.

Yushmanov, P., R.J. Groebner, L.L. Lao, T.N. Carlstrom, "A Scaling for the H-mode Edge Pedestal in DIII-D,” Bull. Am. Phys. Soc. 41, 1575 (1996) Abstract.

Zhang, Daqing, John Lohr, J.W. Tooker, Dan Ponce, R.W. Callis, "Power Accountability with $1 \mathrm{MW} 110 \mathrm{GHz}$ Gyrotron System on the DIII-D Tokamak," Bull. Am. Phys. Soc. 41, 1429 (1996) Abstract. 